\title{
Annotated checklist of fishes from Monterey Bay National Marine Sanctuary with notes on extralimital species
}

\author{
Erica J. Burton', Robert N. Lea ${ }^{2,3}$ \\ I Monterey Bay National Marine Sanctuary, National Ocean Service, National Oceanic and Atmospheric Ad- \\ ministration, 99 Pacific Street, Building 455A, Monterey, California 93940, USA 2 Department of Ichthyo- \\ logy, California Academy of Sciences, Golden Gate Park, 55 Music Concourse Drive, San Francisco, California \\ 94118, USA 3 Section of Ichthyology, Natural History Museum of Los Angeles County, 900 Exposition Boule- \\ vard, Los Angeles, California, 90007, USA \\ Corresponding author: Erica J. Burton (Erica.Burton@noaa.gov)
}

Academic editor: Kyle Piller | Received 5 July 2019 | Accepted 18 September 2019 | Published 7 November 2019

http://zoobank.org/CE659523-94CA-4E87-B131-E863E691D723

Citation: Burton EJ, Lea RN (2019) Annotated checklist of fishes from Monterey Bay National Marine Sanctuary with notes on extralimital species. ZooKeys 887: 1-119. https://doi.org/10.3897/zookeys.887.38024

\begin{abstract}
Monterey Bay National Marine Sanctuary is a federal, marine protected area located off the central coast of California, USA. Understanding biodiversity, and how it is changing, is necessary to effectively manage the sanctuary. The large size of this sanctuary, which contains a variety of habitats and is influenced by several water masses, provides for a diverse fish fauna. The central California coast has a rich history of ichthyological research and surveys, contributing to a unique repository of information on fish diversity. Herein, we provide a checklist of fishes that occur within the sanctuary, including justification for each species. Ancillary record information including name-bearing type specimens, historic species, cold- or warm-water event species, introduced species, and occurrence at Davidson Seamount or Elkhorn Slough are also provided. This represents the first comprehensive annotated checklist of 507 fishes known to occur within the sanctuary. In addition, 18 species are considered to be extralimital. This annotated checklist of fishes can be used by those interested in zoogeography, marine protected areas, ichthyology, regional natural history, and sanctuary management.
\end{abstract}

\section{Keywords}

central California, cold-water event, Davidson Seamount, Elkhorn Slough, El Niño/Southern Oscillation, extralimital, introduced species, La Niña, marine protected area, species inventory, taxonomy, warm-water event

Copyright Erica J. Burton, Robert N. Lea. This is an open access article distributed under the terms of the Creative Commons Attribution License (CC BY 4.0), which permits unrestricted use, distribution, and reproduction in any medium, provided the original author and source are credited. 


\section{Table of contents}

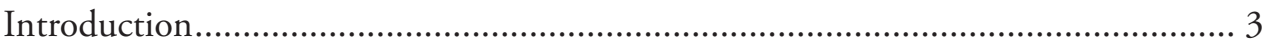

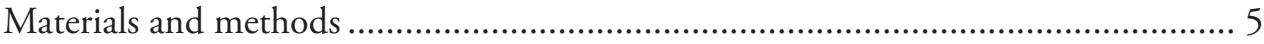

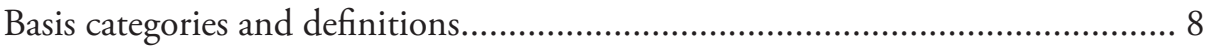

Ancillary record categories and definitions .................................................... 8

Occurrence of note and definitions .............................................................. 9

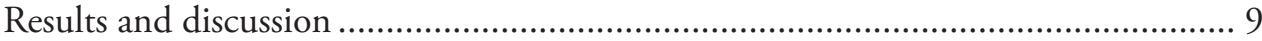

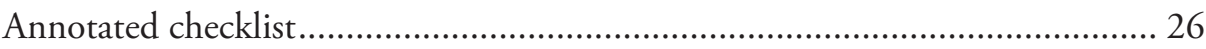

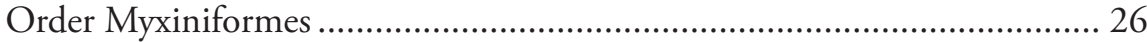

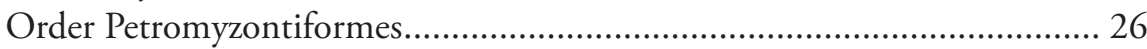

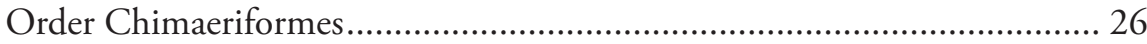

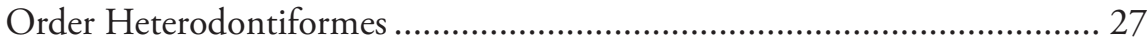

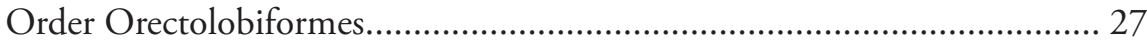

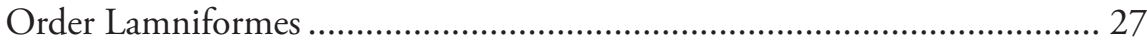

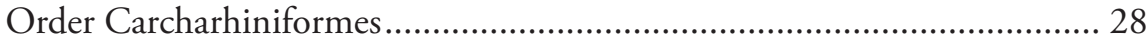

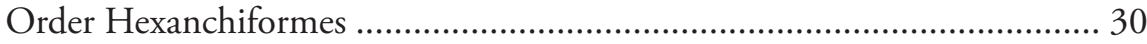

Order Squaliformes................................................................................ 30

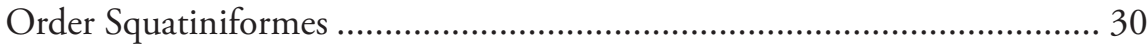

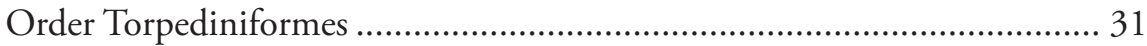

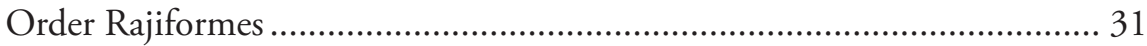

Order Myliobatiformes ........................................................................... 33

Order Acipenseriformes ....................................................................... 34

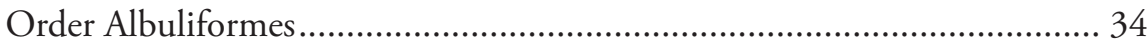

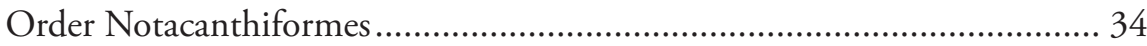

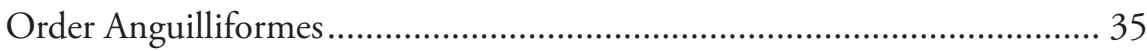

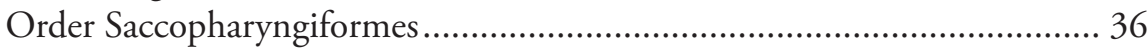

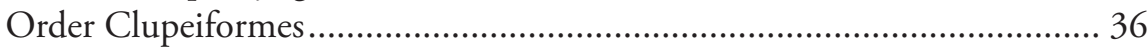

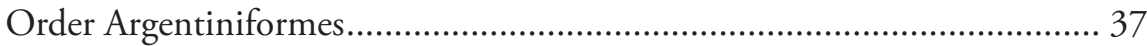

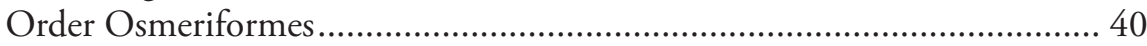

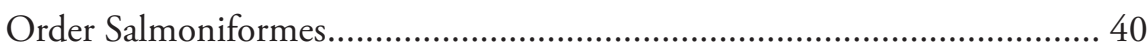

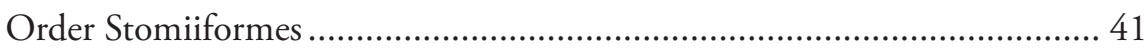

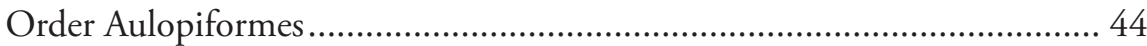

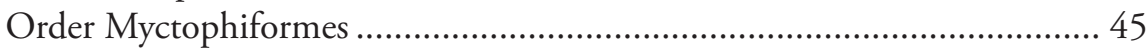

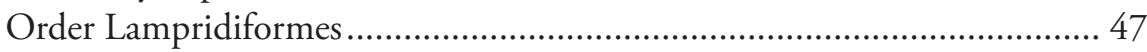

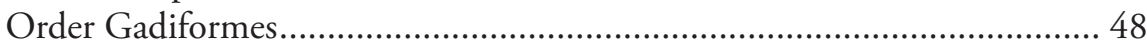

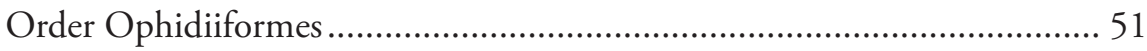

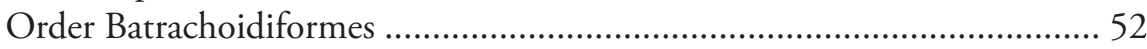

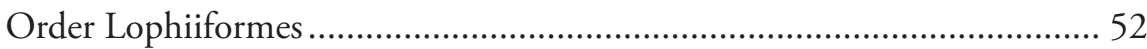

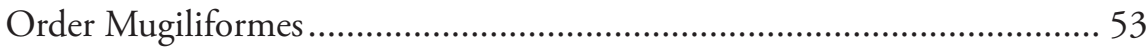

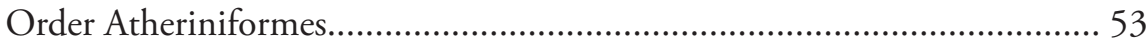

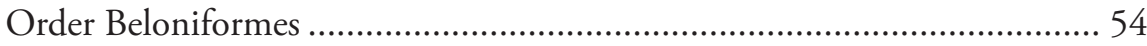

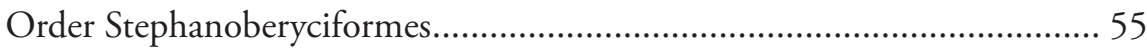




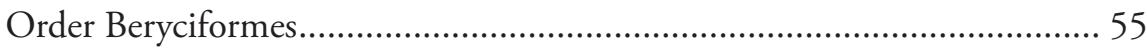

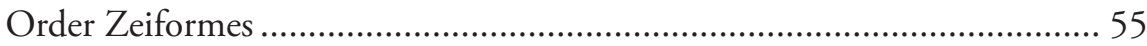

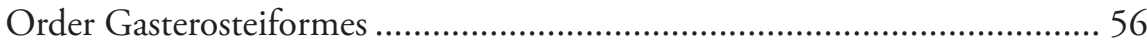

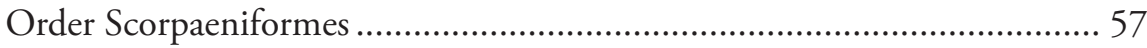

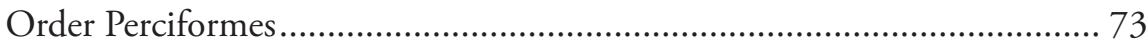

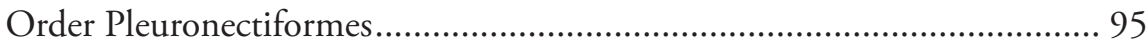

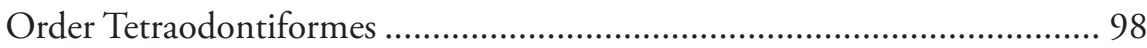

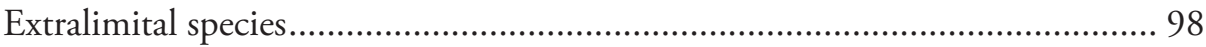

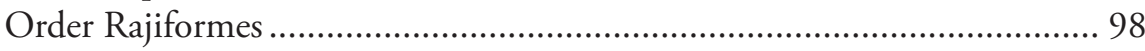

Order Stomiiformes ................................................................................. 99

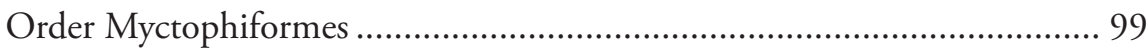

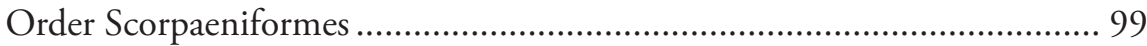

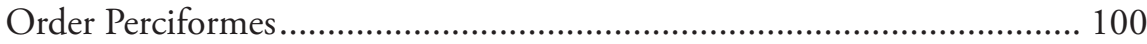

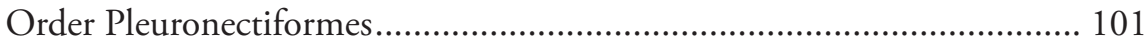

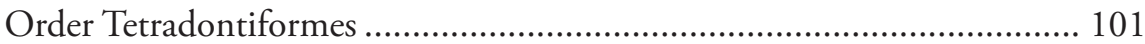

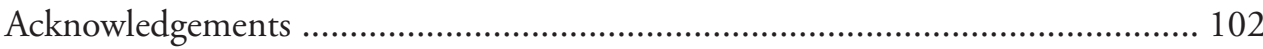

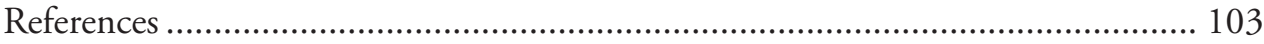

\section{Introduction}

Monterey Bay National Marine Sanctuary (MBNMS or sanctuary) is a federal, marine protected area located off the central coast of California, USA (Fig. 1). Spanning the area from Rocky Point (Marin County) to Cambria (San Luis Obispo County), it encompasses $444 \mathrm{~km}$ of shoreline, 4,601 square $\mathrm{nm}$ of ocean, and extends from mean high tide to a seaward boundary that averages $48 \mathrm{~km}$ offshore. At its deepest point within the Davidson Seamount Management Zone, MBNMS reaches a depth of 3,875 m. The sanctuary contains a variety of habitats including estuaries (largest is Elkhorn Slough), sandy beaches, rocky shores, kelp forests, continental shelf and slope, deep submarine canyons (largest is Monterey Canyon), open ocean, and Davidson Seamount (Fig. 1). These habitats, individually and collectively, harbor an incredible variety of marine life.

It is an inconvenient truth that managing areas for ecosystem sustainability is impossible without knowledge of the species that compose it (Wilson 2014). Moreover, to demonstrate ecological significance or from simple curiosity the question is often asked, "How many species occur in Monterey Bay National Marine Sanctuary?” It may be specific to particular taxa, such as algae, invertebrates, fishes, seabirds and shorebirds, or marine mammals. The answer, however, is not absolute. Species distributions are dynamic (Lonhart 2009) shifting over time due to natural and/or anthropogenic mechanisms, or due to oceanic and atmospheric events (e.g., El Nińo/Southern Oscillation, global change). In addition, species continue to be discovered and described from less sampled areas (e.g., deep sea).

The large size of MBNMS, influenced by several water masses and its proximity to Point Conception, provides for a diverse fish fauna. The central California marine fish 


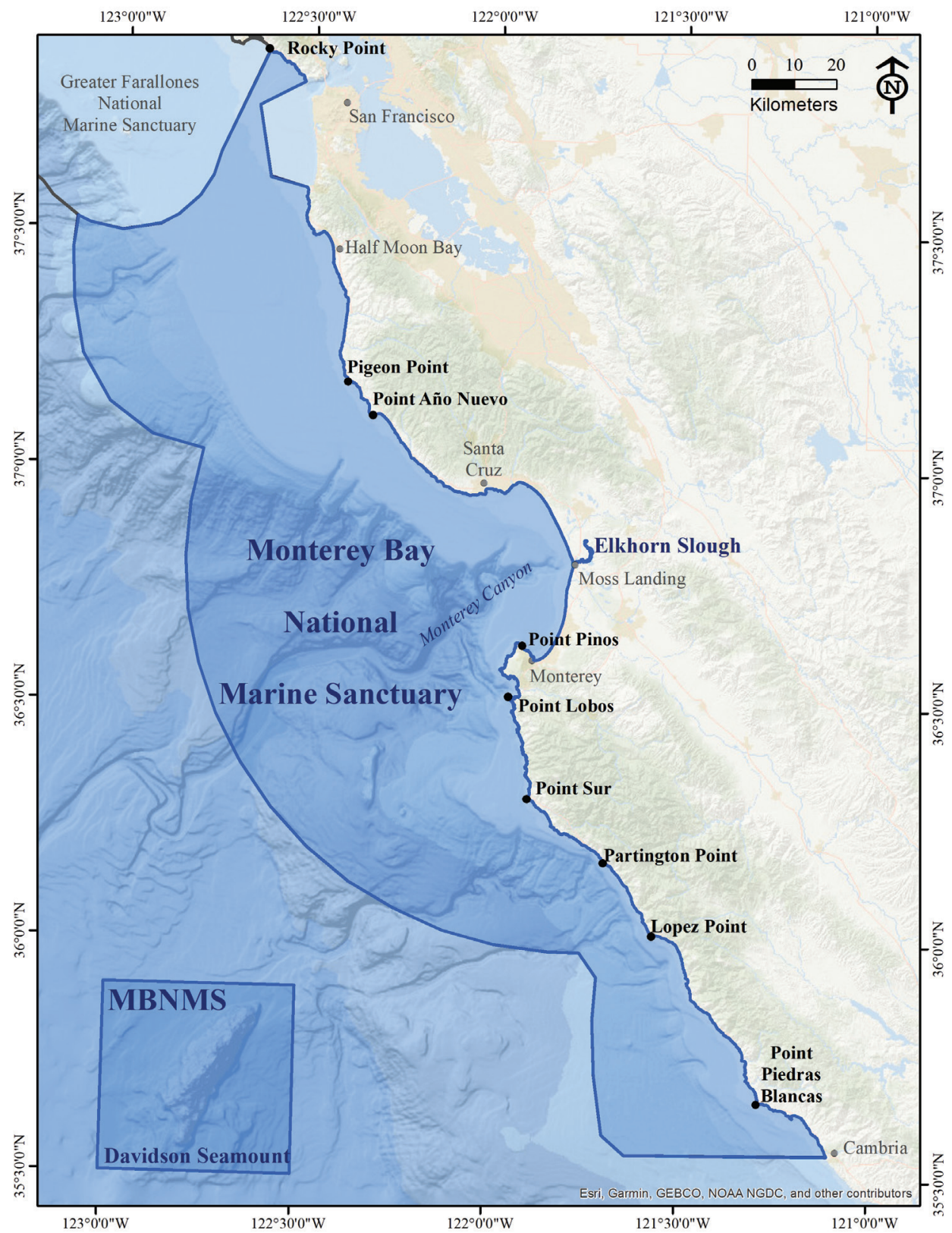

Figure I. Monterey Bay National Marine Sanctuary. Monterey Bay National Marine Sanctuary (MBNMS) is a federal, marine protected area that spans from Rocky Point (Marin County, just north of the Golden Gate Bridge) to Cambria (San Luis Obispo County), California, USA; and includes Elkhorn Slough and the Davidson Seamount Management Zone. Points of interest (bold black font) and cities of interest (light gray font) are notated. Credit: Sophie De Beukelaer (Lynker Technologies LLC/MBNMS). 
fauna is influenced by water masses from the north, south, and west; and these water masses converge to create a major transition zone centered around Point Conception (south of MBNMS, Horn et al. 2006). Two distinctive fish faunas intermingle in this transition zone, a warm-temperate, southern component (San Diegan Province) and a cool-temperate, northern component (Oregonian Province). The fish fauna in the southern end of the sanctuary may at times be influenced by this transition zone, especially during warm-water events such as El Niño/Southern Oscillation. The Davidson Seamount lies well offshore (ca. $121 \mathrm{~km}$ due west of San Simeon, San Luis Obispo County), and the fish fauna there may be influenced by oceanic water masses to the west, such as the Subarctic-Transitional and Central Pacific water masses.

The central California coast has a rich history of ichthyological research and surveys, including: early biological expeditions to describe the California fish fauna (e.g., Pacific Expeditions of the US Fish Commission Steamer "Albatross", Pacific Railroad Surveys); early institutions involved in specimen collections and subsequent study (e.g., California Academy of Sciences, Stanford University, California Department of Fish and Game, currently California Department of Fish and Wildlife, and Scripps Institution of Oceanography); and the more recent expansion of marine research institutions in central California. These early and recent surveys, preserved specimen collections, and scientific publications provide a wealth of information to create an inventory of fishes that occur within MBNMS.

A comprehensive inventory of fish species occurring within MBNMS is unavailable. Disparate lists are available for subsets of fishes, including commonly occurring species, fished species, and species within a particular habitat of MBNMS (e.g., Guerrero and Kvitek 1996, Monterey Bay Aquarium 1999, Starr et al. 2002, Yoklavich et al. 2002, Burton and Lundsten 2008, Burton et al. 2017, SIMoN 2019). We created a comprehensive annotated checklist of fishes occurring within MBNMS based on critical analyses of material from ichthyological collections at natural history museums, the literature, and visual records. The checklist presented here provides sources of basis (i.e., justification), occurrence of fishes during cold- or warm-water events, records of historically occurring fishes, reference to original species descriptions from within MBNMS, special places of occurrence (i.e., Elkhorn Slough, Davidson Seamount), and introduced species. Our goal was to compile a defensible checklist of fishes known to occur within the sanctuary.

\section{Materials and methods}

We first generated a draft list of fishes that should occur within MBNMS based on regional guidebooks and checklists of fishes, including: Fitch and Lavenberg (1968), Miller and Lea (1972), Hubbs et al. (1979), Eschmeyer et al. (1983), Love et al. (2002), Yoklavich et al. (2002), Ebert (2003), Love et al. (2005), and Burton and Lundsten (2008). The majority of these guidebooks encompass broader regions (e.g., 
California, eastern North Pacific) than investigated here. From these lists, we examined basis within MBNMS for each species.

In an effort to provide defensible justification for each species, we considered the following sources as basis for inclusion, in order of importance: 1) museum specimen; 2) publication; and/or 3) expert-verified visual record. In addition, we followed a general rule for inclusion: the occurrence of any life history stage of the species within the current boundaries of MBNMS (including warm-water event, cold-water event, or historic record).

To determine a basis for inclusion (if any), we investigated ichthyological collections at natural history museum collections with online databases including California Academy of Sciences, Stanford University, Scripps Institution of Oceanography at University of California San Diego, Los Angeles County Museum of Natural History, Burke Museum at University of Washington, and the Smithsonian Institution's National Museum of Natural History. In addition, we consulted the Moss Landing Marine Laboratories fish collection, which is primarily a teaching resource. Where records were few and/or questionable, we examined specimens that were readily accessible to confirm identification. If museum specimens were lacking, we then consulted publications, where primary sources of peer-reviewed scientific literature carried the most weight. Finally, if taxonomic experts could verify species from imagery (i.e., photos or video), we sparingly used these visual record(s) for basis. With technological advancements, video observations of deep-sea species are on the rise. Monterey Bay Aquarium Research Institute's (MBARI) online Deep Sea Guide (Jacobson Stout et al. 2017) provides an excellent source of in situ imagery observations of regional species, particularly for rarely collected deep-sea species.

In addition, we conducted a midwater trawl survey during May 2015 aboard NOAA Ship "Bell M. Shimada", which included the following areas of MBNMS: Davidson Seamount, Sur Ridge, Monterey Canyon, and off Monterey (unpublished data, Burton, Lea, and DeVogelaere). Juvenile and adult specimens were deposited at California Academy of Sciences (CAS), and larval specimens were stored as uncatalogued specimens at Southwest Fisheries Science Center (SWFSC), National Marine Fisheries Service (NMFS), La Jolla, California.

The checklist is organized in taxonomic order, following "Fishes of the World" (Nelson 2006), unless otherwise stated. Species names are alphabetized within family. Authority is included following current usage in "Catalog of Fishes" (Eschmeyer et al. 2017) and "Common and Scientific Names of Fishes" (Page et al. 2013), unless otherwise stated. If a species was originally described from within MBNMS boundaries, the original publication (therefore authority) is included in the references section (as basis). Official common names follow Page et al. (2013) or Hubbs et al. (1979), unless otherwise stated; and are capitalized following Page et al. (2013). Where no official common name was available, a generic common name (lowercase) was used, based on family name.

Terms used to describe species inclusion are defined below. Symbolic museum codes used in the checklist are listed in Table 1; full museum names and location information were sourced from Sabaj (2016). Other institution codes and acronyms are listed in Table 2. 
Table I. Symbol codes for natural history museum collections, including names of institutions and locations. Source: Sabaj (2016).

\begin{tabular}{|c|c|c|}
\hline Abbrev. & Name & Location \\
\hline ANSP & Academy of Natural Sciences of Drexel University & Philadelphia, Pennsylvania, USA \\
\hline BMNH & Natural History Museum [formerly British Museum (Natural History)] & London, England, UK \\
\hline CAS-ICH & California Academy of Sciences, Ichthyology Collection & San Francisco, California, USA \\
\hline CAS-SU & $\begin{array}{l}\text { Stanford University (collection now housed at California Academy of } \\
\text { Sciences) }\end{array}$ & San Francisco, California, USA \\
\hline FMNH & Field Museum of Natural History, Zoology Department & Chicago, Illinois, USA \\
\hline KU & University of Kansas Biodiversity Institute & Lawrence, Kansas, USA \\
\hline LACM & Natural History Museum of Los Angeles County & Los Angeles, California, USA \\
\hline MBARI & Monterey Bay Aquarium Research Institute & Moss Landing, California, USA \\
\hline MCZ & Museum of Comparative Zoology, Harvard University & Cambridge, Massachusetts, USA \\
\hline MLMLF & Moss Landing Marine Laboratories Fishes & Moss Landing, California, USA \\
\hline MNHN & Muséum national d'Histoire naturelle, Paris & Paris, France \\
\hline NCSM & $\begin{array}{l}\text { North Carolina Museum of Natural Sciences [formerly North Carolina } \\
\text { State Museum] }\end{array}$ & Raleigh, North Carolina, USA \\
\hline OS & Oregon State University, Department of Fisheries and Wildlife & Corvalis, Oregon, USA \\
\hline OSUM & $\begin{array}{l}\text { Ohio State University, Museum of Biological Diversity, Museum of } \\
\text { Zoology }\end{array}$ & Columbus, Ohio, USA \\
\hline SBMNH & Santa Barbara Museum of Natural History & Santa Barbara, California, USA \\
\hline SIO & Scripps Institution of Oceanography at University of California San Diego & La Jolla, California, USA \\
\hline UAM & University of Alaska Museum of the North, Fairbanks & Fairbanks, Alaska, USA \\
\hline UCLA & University of California Los Angeles (now at SIO) & Los Angeles, California, USA \\
\hline UCM & University of Colorado Museum of Natural History, Boulder, Colorado & Boulder, Colorado, USA \\
\hline UF & University of Florida, Florida Museum of Natural History & Gainesville, Florida, USA \\
\hline UMMZ & University of Michigan Museum of Zoology & Ann Arbor, Michigan, USA \\
\hline USNM & $\begin{array}{l}\text { National Museum of Natural History, Smithsonian Institution [formerly } \\
\text { US National Museum] }\end{array}$ & Washington, DC, USA \\
\hline UW & University of Washington & Seattle, Washington, USA \\
\hline ZMB & Museum für Naturkunde [formerly Zoologischen Museum] & Berlin, Germany \\
\hline ZMUB & $\begin{array}{l}\text { Universitetet Bergen, Bergen Museum, Naturhistorie, Zoologiske, } \\
\text { Vertebratsamlinger }\end{array}$ & Bergen, Norway \\
\hline
\end{tabular}

Table 2. List of institution codes (non-museum) and other acronyms.

\begin{tabular}{ll}
\hline \multicolumn{1}{c}{ Acronym } & \multicolumn{1}{c}{ Institution or definition } \\
\hline CalCOFI & California Cooperative Oceanic Fisheries Investigations \\
CDFG & California Department of Fish and Game (now CDF Wildlife) \\
CSUMB & California State University Monterey Bay \\
ESNERR & Elkhorn Slough National Estuarine Research Reserve \\
IfAME & Institute for Applied Marine Ecology (at CSUMB) \\
MBA & Monterey Bay Aquarium \\
MBARI & Monterey Bay Aquarium Research Institute \\
MBNMS & Monterey Bay National Marine Sanctuary \\
MLML & Moss Landing Marine Laboratories \\
NOAA & National Oceanic and Atmospheric Administration \\
NMFS & National Marine Fisheries Service \\
SIMoN & Sanctuary Integrated Monitoring Network \\
SL & Standard Length \\
SWFSC & Southwest Fisheries Science Center (at NMFS) \\
TL & Total Length \\
UCLA & University of California, Los Angeles \\
\hline
\end{tabular}




\section{Basis categories and definitions}

Museum specimens: Specimens were collected within MBNMS and catalogued at a natural history museum. Specimens listed do not necessarily represent all catalogued specimens occurring within MBNMS, but merely as examples to provide a basis for inclusion. We attempted to list three records (where available); and for those records to span a geographical range of occurrence within MBNMS, identified by known experts. Records are listed in alphabetical order, according to museum symbolic code (list of natural history museum collections and symbolic codes used for this checklist are provided in Table 1); type specimens are listed first. Collection location and name of who identified specimen are included. Identifier's first initial, middle initial (if provided), and last name are provided; names are transcribed as provided and no assumptions are made to refine or match to others. Year of collection may be noted, especially if species occurred during an unusual oceanographic event. Type specimens (name-bearing and non-name-bearing) may be denoted (see ancillary record categories, below). Museum specimen(s) notated in checklist as follows: Museum code and catalog number (collection location, identifier).

Publications: Peer-reviewed information that provides a basis for occurrence within MBNMS. Other supporting publications may also be listed. Location observed is in parentheses. Year of collection may be noted, especially if species occurred during an unusual oceanographic event. Publication(s) notated in checklist as follows: Author (year) (location, additional information if available).

Visual records: Species observed with imagery (video or photo) and verified by expert or authority. Year of collection may be noted, especially if it occurred during an unusual oceanographic event. Visual record(s) are listed in checklist as institution code and dive number (observed location, identifier).

Several species occurring within MBNMS are anadromous (i.e., move up streams and rivers from the sea to spawn). Records of species occurring in fresh or brackish water (adjacent to MBNMS) are included where no marine records are available, because part of the life cycle requires movement between fresh and marine water bodies, thereby traversing MBNMS waters.

\section{Ancillary record categories and definitions}

Type specimens: Name-bearing type specimens (holotype, neotype, syntype, or lectotype) collected within MBNMS. Type specimen(s) are listed in checklist as namebearing type (museum code and catalog number).

Historic specimens: Specimen(s) collected many years before present, but not known from recent years; and not otherwise collected during unusual oceanographic events (i.e., warm or cold-water events).

Warm-water events: Specimen(s) collected during, or soon after, unusually warmwater events (e.g., warm phase of El Niño/Southern Oscillation, ENSO; El Niño), but otherwise unknown or rare during normal oceanographic conditions. Several refer- 
ences were consulted to determine the occurrence of warm-water events, including Wolter and Timlin (2011) (ENSO behavior since 1871), NOAA Earth System Research Laboratory (2019), and Null (2019); referencing the Multivariate ENSO Index (MEI), Oceanic Niño Index (ONI), and Niño 3.4 region.

Cold-water events: Specimen(s) collected during, or soon after, unusually coldwater events (e.g., cold phase of El Niño/Southern Oscillation: La Niña), but otherwise unknown or rare during normal oceanographic conditions. Several references were consulted to determine the occurrence of cold-water events, including Wolter and Timlin (2011) (ENSO behavior since 1871), NOAA Earth System Research Laboratory (2019), and Null (2019), referencing the Multivariate ENSO Index (MEI), Oceanic Niño Index (ONI), and Niño 3.4 region.

\section{Occurrence of note and definitions}

Davidson Seamount area: Known to occur within the Davidson Seamount Management Zone of MBNMS (Fig. 1).

Elkhorn Slough area: Known to occur in Elkhorn Slough (within MBNMS, Fig. 1) or adjacent areas (i.e., Moss Landing Harbor, jetties).

Introduced species: Non-native to MBNMS, and either accidentally or deliberately transported to the area.

Extralimital: Species known to occur from areas to the north and south of MBNMS, but no basis could be found within MBNMS (yet likely to occur within MBNMS). Extralimital species are not considered part of the primary checklist, and are listed separately.

\section{Results and discussion}

The annotated checklist includes 507 fish species, representing 325 genera and 148 families (Table 3). The number of taxa supported by specific sources of basis are as follows: museum specimens (465); publications (230); and visual records (44). Original descriptions of 57 species (type specimens, 11\%) originated from within current MBNMS boundaries.

Seven species are considered historic: Pink Salmon (Oncorhynchus gorbuscha); Chum Salmon (Oncorhynchus keta); Spotted Sand Bass (Paralabrax maculatofasciatus); Salema (Haemulon californiensis); California Corbina (Menticirrhus undulatus); Garibaldi (Hypsypops rubicundus); and Pacific Sandfish (Trichodon trichodon). These species have not been observed within MBNMS for many years, and/or have rarely been observed. Their recent rarity can be attributed to local extinction (i.e., salmon), or otherwise a northern or southern species that has rarely been observed in sanctuary waters. Although rarely observed or not observed for some time, they could potentially occur again and remain on the checklist. 
At least 50 species are known to occur during unusual oceanographic events (e.g., warm or cold phases of El Niño/Southern Oscillation). The 49 species occurring during warm-water events do not necessarily occur solely during warm-water events (e.g., El Niño), but they are more likely to be observed at these times (see Table 3). The occurrence of the Whale Shark (Rhincodon typus) was reported during several cold-water events (La Niña, Ebert et al. 2004). These observations seem counterintuitive, as $R$. typus typically occupies tropical waters (Miller and Lea 1972, Ebert 2003, Love et al. 2005, Castro 2011). We include the cold-water categorization here, as reported by Ebert et al. (2004).

Species occurring within two specific habitats of the sanctuary, Elkhorn Slough and Davidson Seamount, are noted due to the uniqueness of each habitat compared to the rest of MBNMS. Of the 507 species in MBNMS, 79 species are found in the Elkhorn Slough area, and 83 species in the Davidson Seamount area. The majority of these species are not restricted to these habitats within MBNMS, although Elkhorn Slough may be the exception. This is not surprising due to the mixture of brackish and saltwater habitats, and the life history requirements of some species. Elkhorn Slough also provides habitat for marine species from nearshore waters to feed, mate, and spawn (Yoklavich et al. 2002). Five species are less likely to occur in marine environments: Threadfin Shad (Dorosoma petenense; introduced species); Threespine Stickleback (Gasterosteus aculeatus); Yellowfin Goby (Acanthogobius flavimanus; introduced species); Arrow Goby (Clevelandia ios); and Longjaw Mudsucker (Gillichthys mirabilis). The Davidson Seamount Management Zone occurs far offshore and the fish fauna there is influenced by oceanic water masses to the north and west, such as the Subarctic-Transitional and Central Pacific water masses. The 83 species occurring at Davidson Seamount are not restricted to the seamount and occur elsewhere in deep-sea or oceanic habitats.

Five species are non-native, that were either purposefully or inadvertently introduced to California: American Shad (Alosa sapidissima); Threadfin Shad (Dorosoma petenense); Striped Bass (Morone saxatilis); Barred Knifejaw (Oplegnathus fasciatus); and Yellowfin Goby (Acanthogobius flavimanus). The Barred Knifejaw is the most recent introduction to MBNMS (2014, one confirmed living specimen), and it remains to be seen if it will establish itself as a viable resident.

Four species are listed with reservation: waryfish (Scopelosaurus adleri); lanternfish (Lampanyctus tenuiformis); Spotted Sand Bass (Paralabrax maculatofasciatus); and California Corbina (Menticirrhus undulatus). Reservation with the first two of these species is due to lack of confidence with identification; the latter two are categorized as historic and/or occurring during warm-water events, and evidence is scant (see checklist for explanation). We include these species on the checklist with the basis as cited.

Eighteen species are considered to be extralimital, are not considered part of the primary checklist, and are listed separately. The geographic ranges for these species encompass MBNMS boundaries. They are likely to occur within MBNMS; however, no verifiable records occur from within MBNMS. These species should be anticipated during future MBNMS surveys and deposited and catalogued at a natural history museum.

In recent years erroneous geographical distributions were introduced in the literature for two species: Basketweave Cusk-eel (Ophidion scrippsae) and Spotted Turbot 
(Pleuronichthys ritteri). Both errors were based on misidentifications in the field that were then perpetuated in the literature. Once these sorts of mistakes occur and are repeated, these myths become difficult to eradicate. Our purpose in emphasizing these examples is to shed light on such problems and resolve these misrepresentations. First, the northern limit for Ophidion scrippsae has been reported as "Central California" (Love et al. 2005; Kells et al. 2016) based on NOAA trawl survey field identifications off the Santa Cruz County coast (lat. 37³8'N, 209 m; Lauth 2001). This represented the first record north of Point Arguello (3434'38”N, 120³9'2”W; 11.75 nautical miles NW of the Point Conception faunal boundary) and from a depth greatly exceeding its known bathymetric limit. The accessioned specimens (UW 47375) were examined by RN Lea and re-determined as Spotted Cusk-eel (Chilara taylori), with both location and depth well within the limits for Chilara taylori. The known northern limit for Ophidion scrippsae is off the vicinity of Point Arguello (SIO 48-304, 3 specimens) and the maximum confirmed depth is $135 \mathrm{~m}$ (off central Baja California). Similarly, the northern limit for Pleuronichthys ritteri was reported off Northern California (Love et al. 2005; Kells et al. 2016) based on field-identified specimens (lat. 3755'N; Weinberg et al. 2002). The accessioned material (UW 47438) was examined by RN Lea and JW Orr (NOAA, NMFS) and re-determined as Hornyhead Turbot (Pleuronichthys verticalis), a species relatively common within MBNMS. Pleuronichthys ritteri is primarily a southern California-Baja California shallow water species (Fitch 1963) with a known northern limit of Morro Bay (LACM 33703-1). We bring these emendations to the attention of the reader to avoid further confusion and note there are no confirmed records of either Ophidion scrippsae or Pleuronichthys ritteri from MBNMS.

This is the first annotated checklist of fishes that occur within Monterey Bay National Marine Sanctuary (see Table 3). All fishes listed here also occur outside of MBNMS boundaries, and full species ranges can be found from other sources (e.g., Miller and Lea 1972, Eschmeyer et al. 1983, Love et al. 2002, Ebert 2003, Love et al. 2005, and Kells et al. 2016).

Species checklists or inventories can be used by various user groups for different reasons. A simple checklist can teach and inform users of what to expect within a geographic area, including educators, students, and those interested in regional natural history. This annotated checklist includes name-bearing type specimen information for students and ichthyologists interested in species discoveries (and original descriptions) that occurred within today's MBNMS boundaries. Those interested in zoogeography of fishes may be interested in historic and recent occurrence information for fishes within MBNMS. In addition, warm-water and cold-water event information is included to help explain potentially temporary occurrence patterns, or sporadic events. An inventory of known sanctuary resources is a basic requirement of the National Marine Sanctuaries Act (NMSA) of 1972 , as amended (16 U.S.C. $\$ 1431$ et seq.). Species inventories provide evidence of occurrence and estimates of species richness. Establishing an inventory is a crucial first step to further identify those species that are endemic, threatened, introduced (provided herein), or socio-economically important, information which may be useful for sanctuary managers. It is our hope that this checklist will be useful for many readers and serve as a model for species inventories for the National Marine Sanctuary System. 
Table 3. Monterey Bay National Marine Sanctuary Fishes Checklist summary. Species are listed in taxonomic order, and include scientific name, authority, and common name. Categories to summarize basis and occurrence are provided, including: Basis for Inclusion (i.e., museum specimen, publication, visual record); Ancillary Record (i.e., type specimen, historic, warm-water event, cold-water event); and Occurrence of Note (i.e., Davidson Seamount area, Elkhorn Slough area, Introduced Species). Symbols denote the following: X (basis provided by); M (museum specimen); P (publication); V (visual record). Column subtotals are provided after line 507. See Methods for explanation of basis.

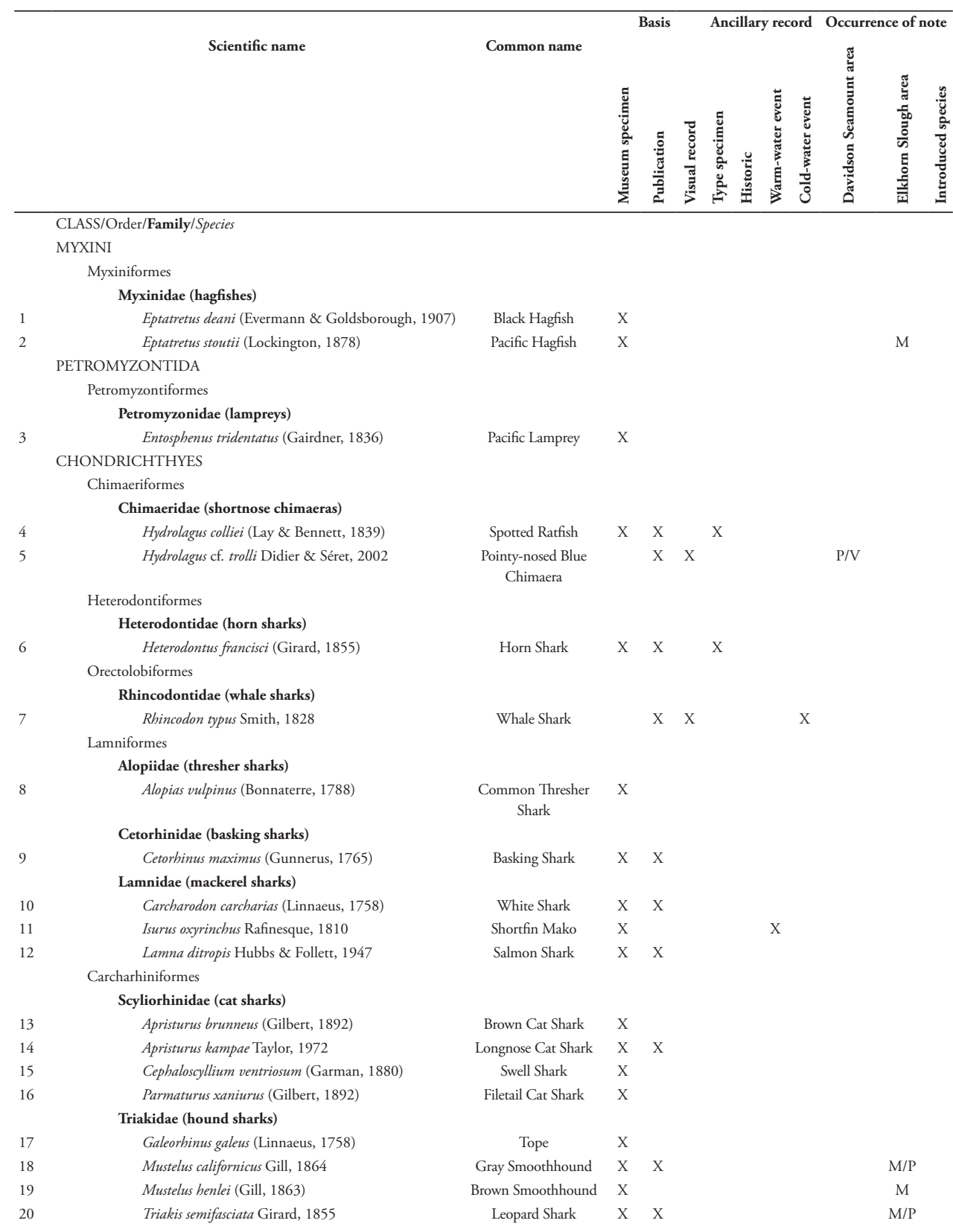




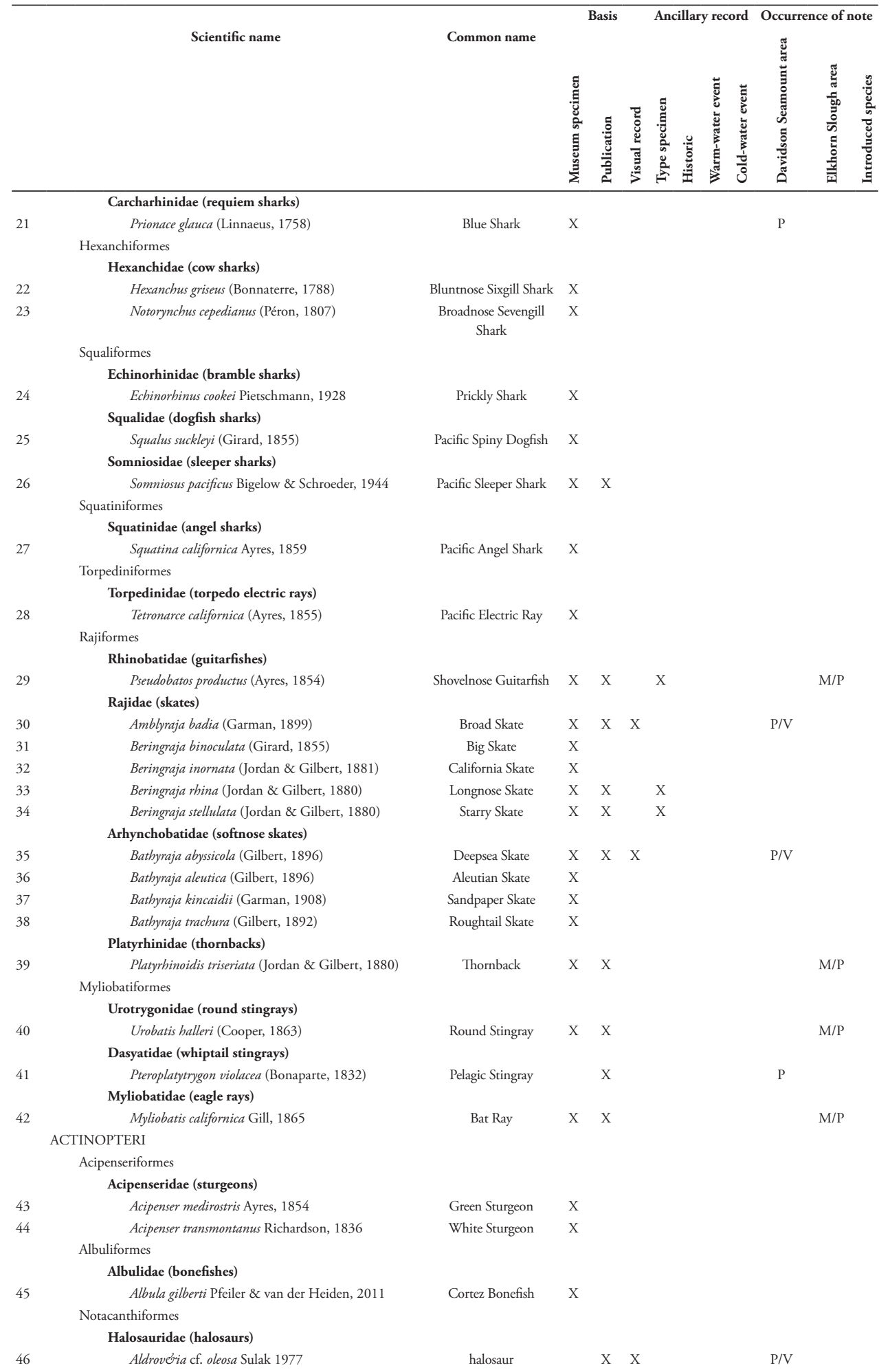




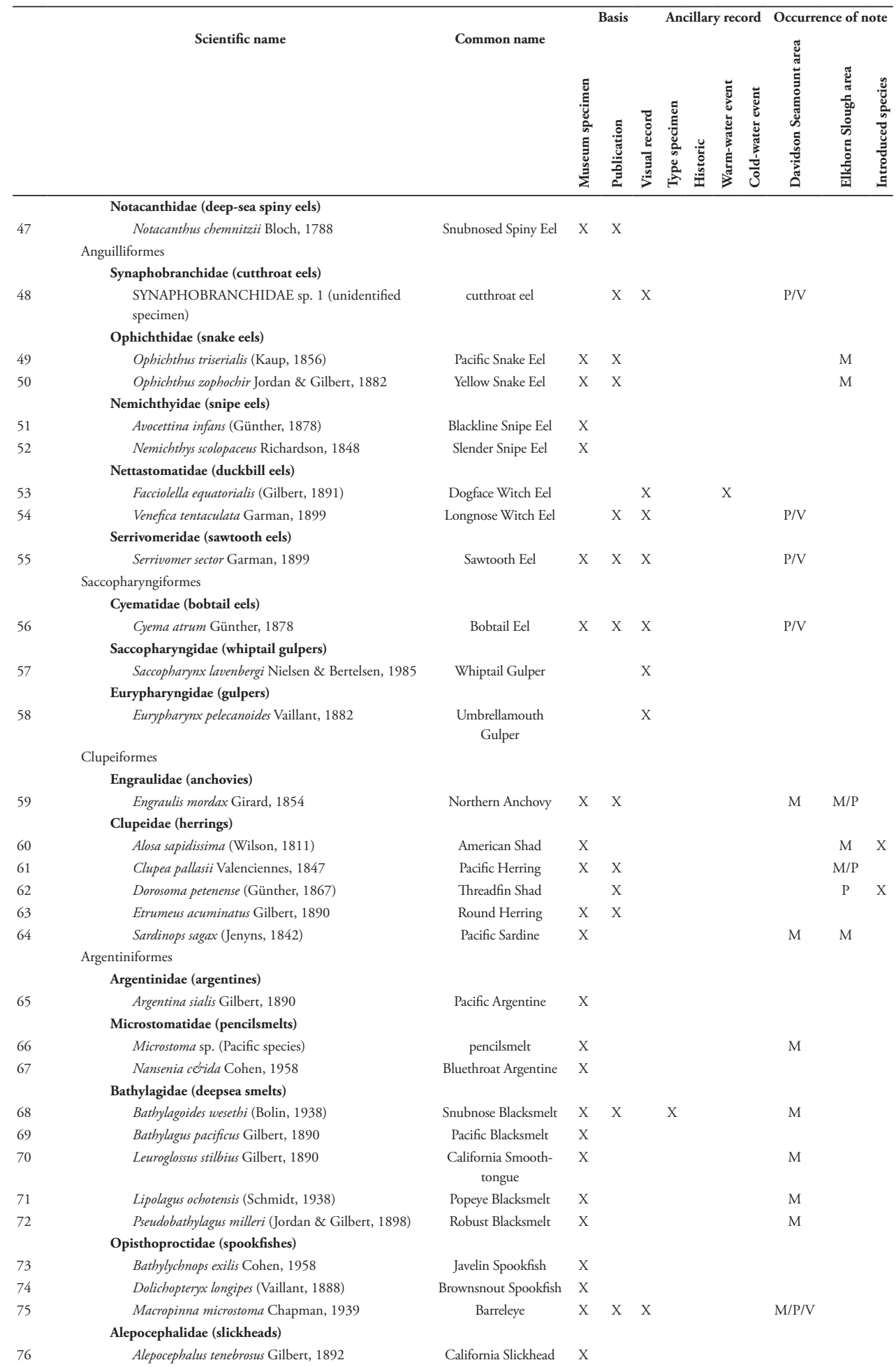




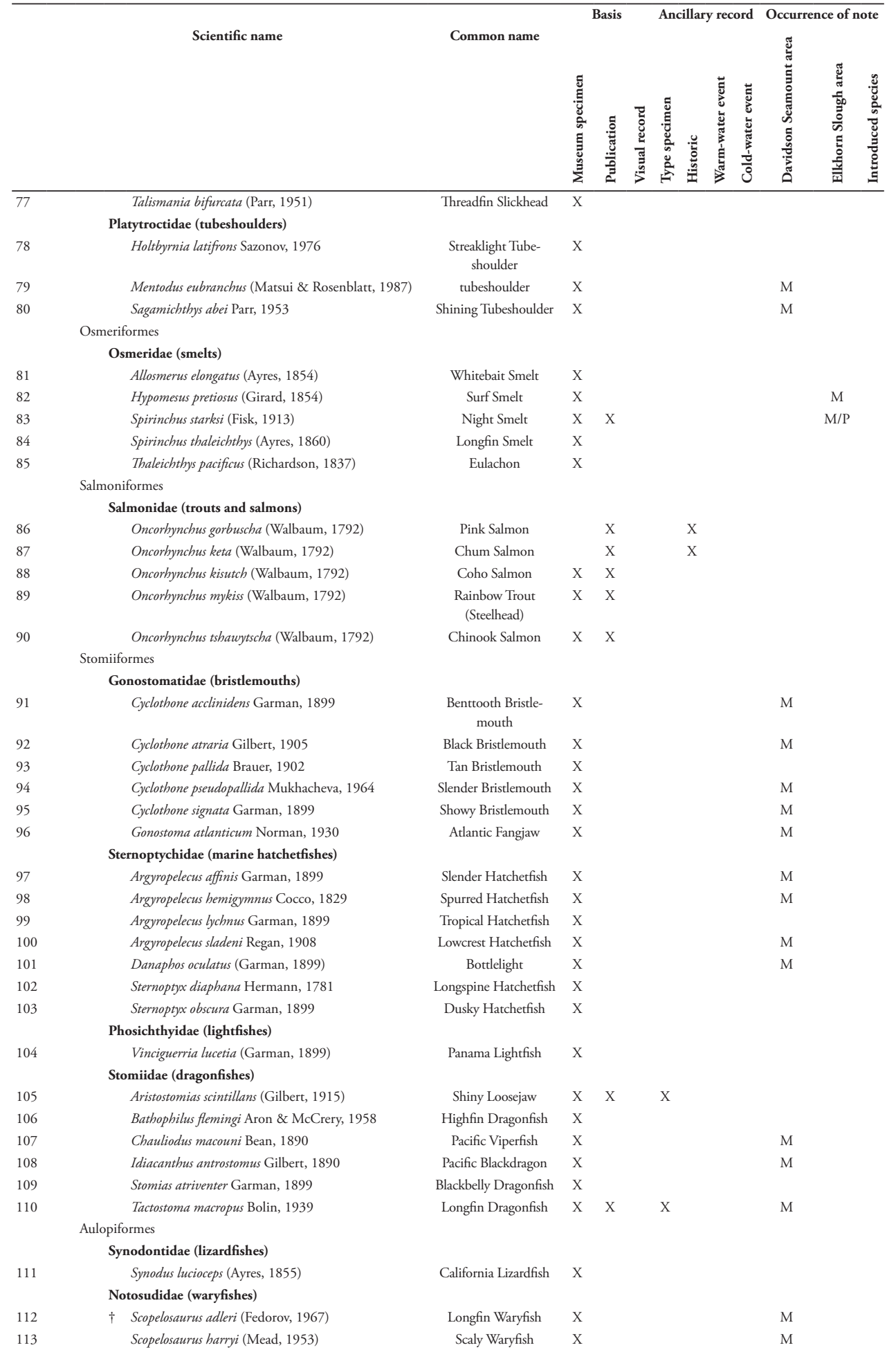




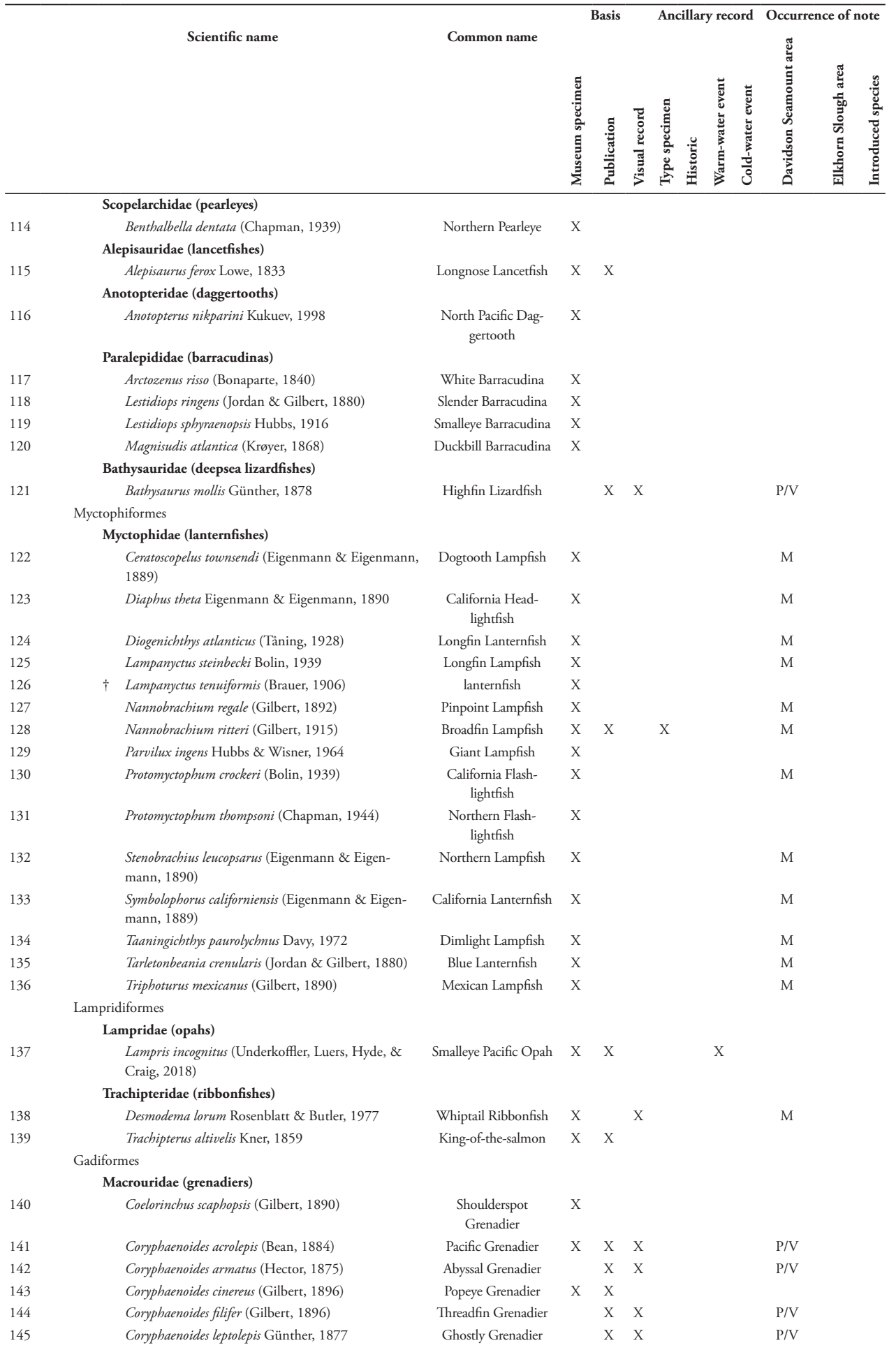




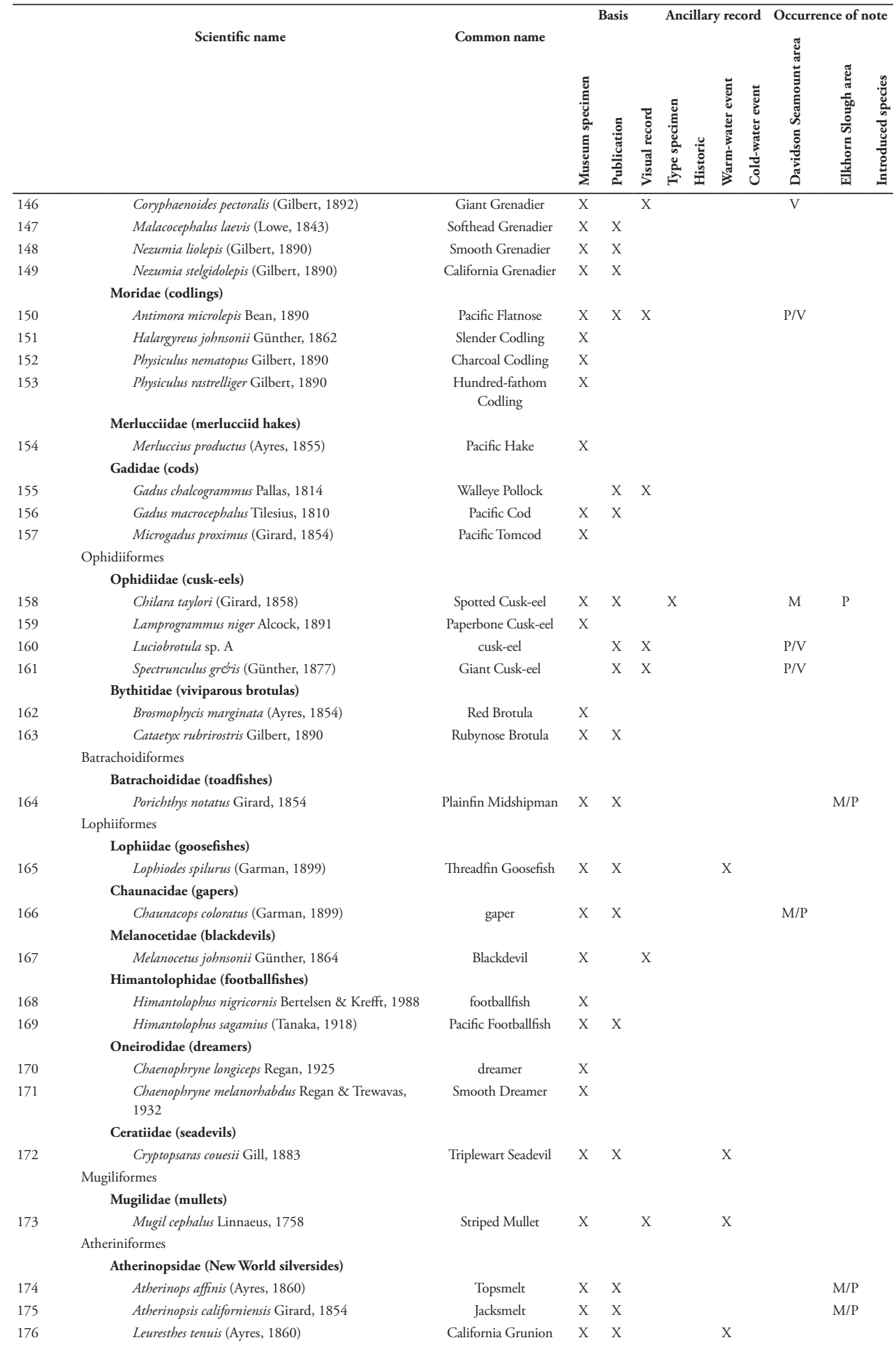




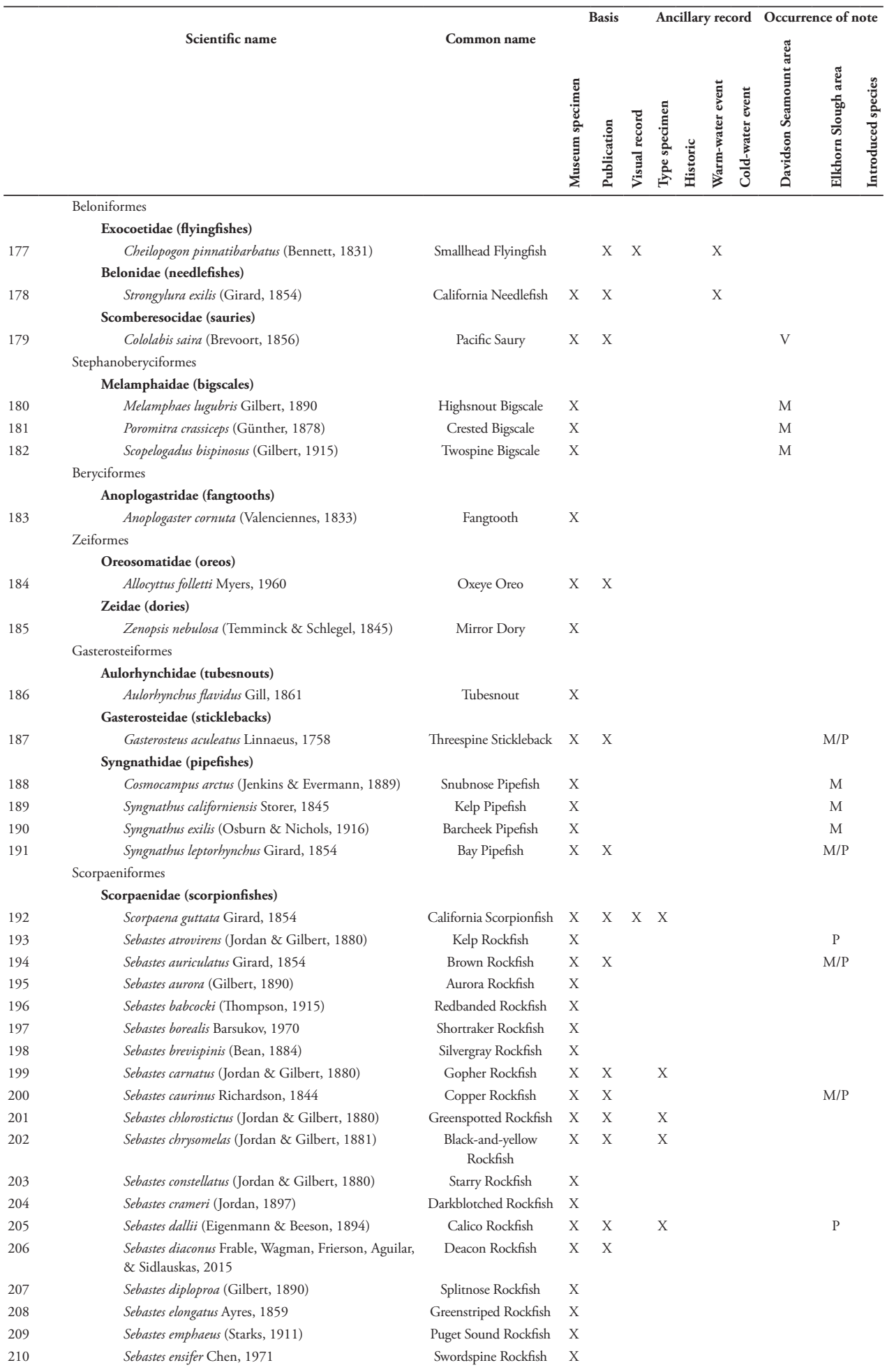




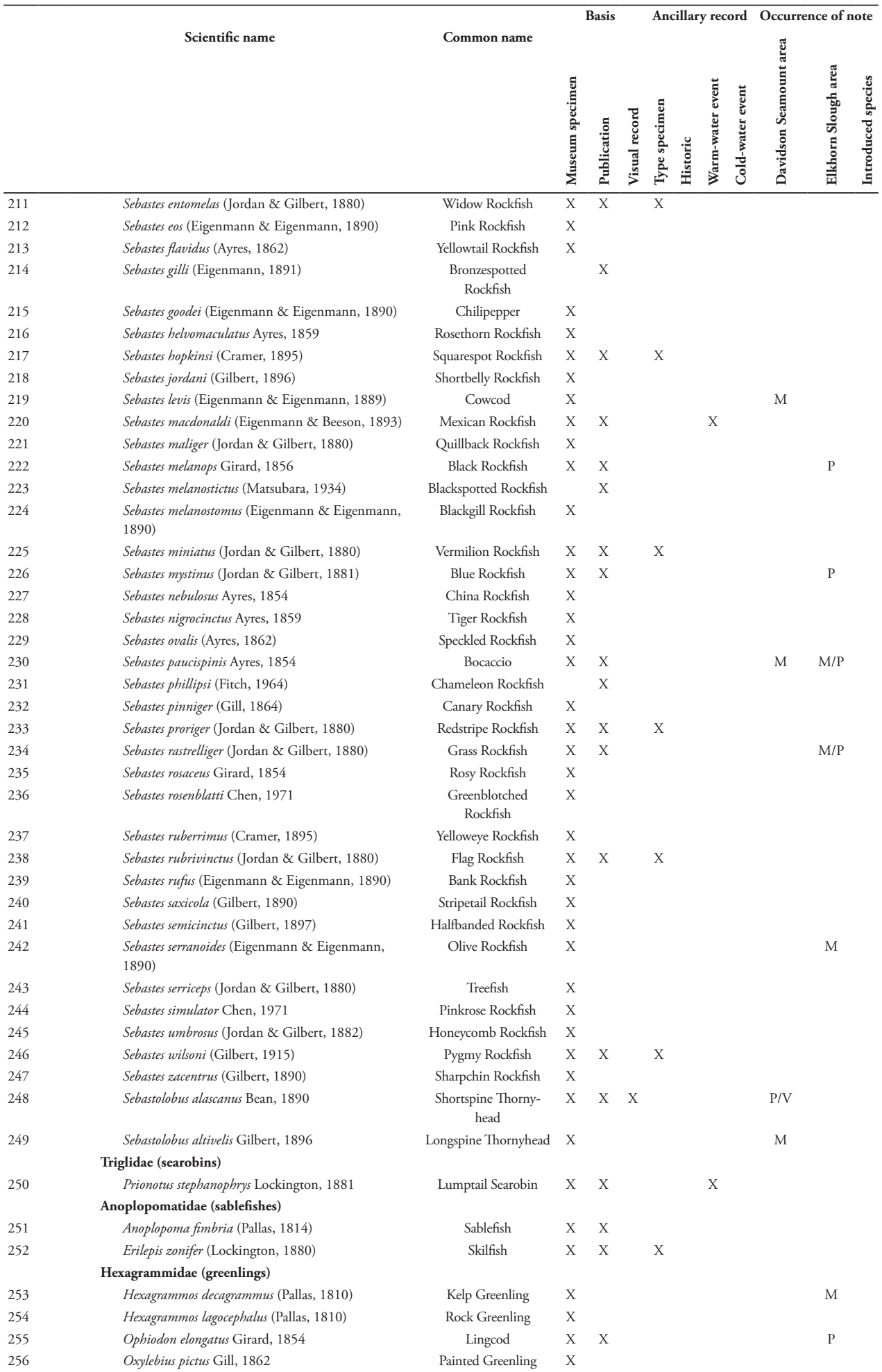




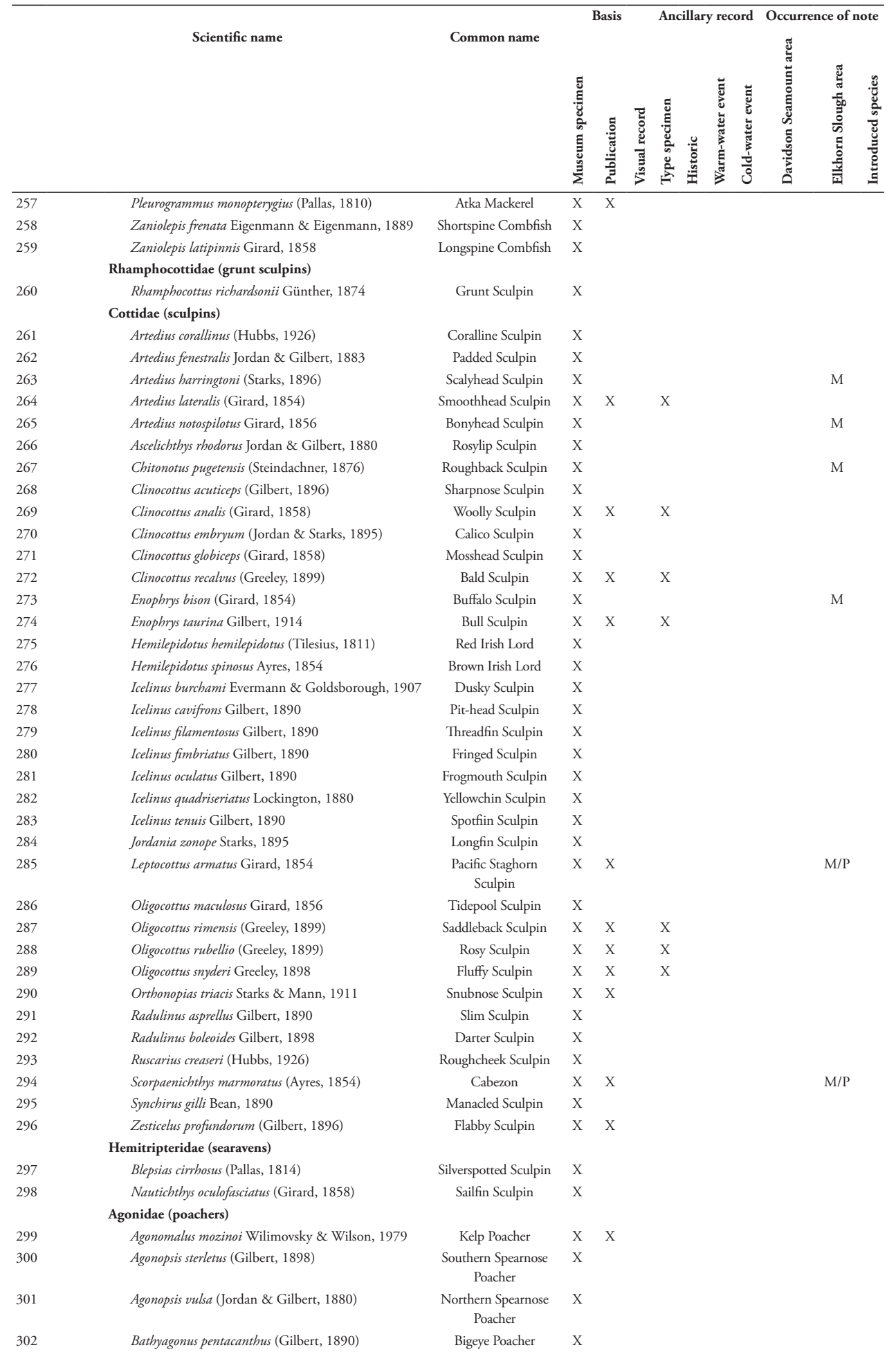




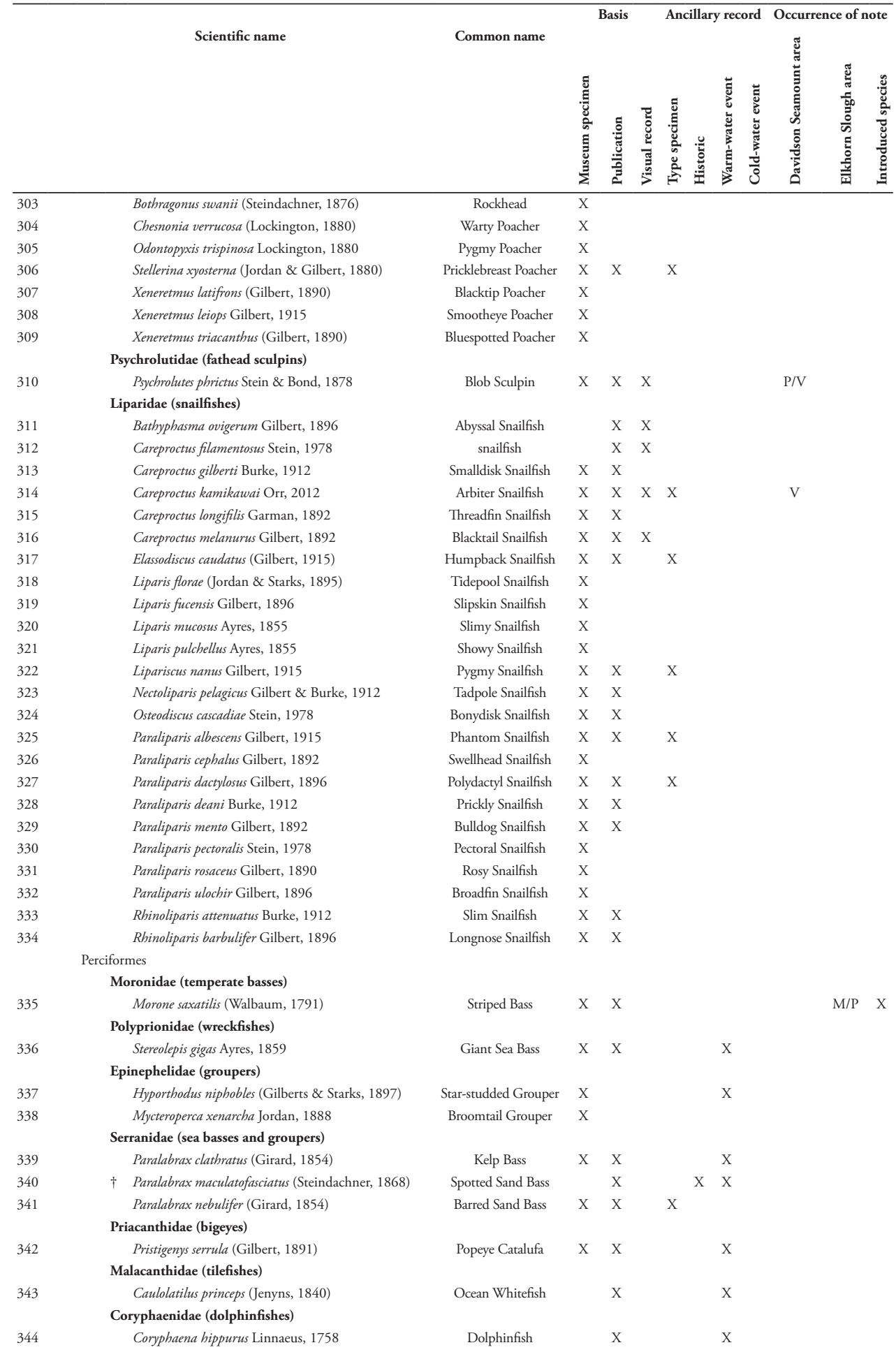




\begin{tabular}{|c|c|c|c|c|c|c|c|c|c|c|c|c|}
\hline & \multirow[b]{2}{*}{ Scientific name } & \multirow[b]{2}{*}{ Common name } & \multicolumn{3}{|c|}{ Basis } & \multicolumn{4}{|c|}{ Ancillary record } & \multicolumn{3}{|c|}{ Occurrence of note } \\
\hline & & & 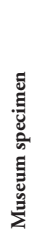 & 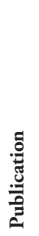 & 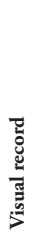 & 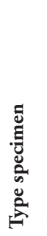 & $\frac{0}{3}$ & 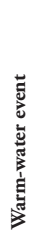 & 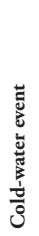 & 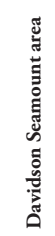 & 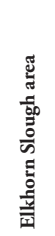 & 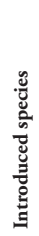 \\
\hline & Echeneidae (remoras) & & & & & & & & & & & \\
\hline 345 & Remora australis (Bennett, 1840) & Whalesucker & $\mathrm{X}$ & & & & & & & & & \\
\hline 346 & Remora remora (Linnaeus, 1758) & Remora & $\mathrm{X}$ & & & & & & & & & \\
\hline & Carangidae (jacks) & & & & & & & & & & & \\
\hline 347 & Caranx caballus Günther, 1868 & Green Jack & $\mathrm{X}$ & $\mathrm{X}$ & & & & $\mathrm{X}$ & & & & \\
\hline 348 & Decapterus muroadsi (Temminck \& Schlegel, 1844) & Amberstripe Scad & $\mathrm{X}$ & $\mathrm{X}$ & & & & & & & & \\
\hline 349 & Naucrates ductor (Linnaeus, 1758) & Pilotfish & $\mathrm{X}$ & & & & & & & & & \\
\hline 350 & Seriola dorsalis (Gill, 1863) & Yellowtail Jack & $\mathrm{X}$ & & & & & & & & & \\
\hline 351 & Trachurus symmetricus (Ayres, 1855) & Jack Mackerel & $\mathrm{X}$ & $\mathrm{X}$ & & & & & & $\mathrm{P}$ & M & \\
\hline & Bramidae (pomfrets) & & & & & & & & & & & \\
\hline 352 & Brama japonica Hilgendorf, 1878 & Pacific Pomfret & $\mathrm{X}$ & & & & & & & & & \\
\hline 353 & Pteraclis aesticola (Jordan \& Snyder, 1901) & Pacific Fanfish & $\mathrm{X}$ & & & & & & & & & \\
\hline & Caristiidae (manefishes) & & & & & & & & & & & \\
\hline 354 & Caristius macropus (Bellotti, 1903) & Bigmouth Manefish & $\mathrm{X}$ & & & & & & & & & \\
\hline & Haemulidae (grunts) & & & & & & & & & & & \\
\hline 355 & Haemulon californiensis (Steindachner, 1876) & Salema & $\mathrm{X}$ & $\mathrm{X}$ & & & $\mathrm{X}$ & $\mathrm{X}$ & & & & \\
\hline & Polynemidae (threadfins) & & & & & & & & & & & \\
\hline 356 & Polydactylus approximans (Lay \& Bennett, 1839) & Blue Bobo & $\mathrm{X}$ & $\mathrm{X}$ & & & & $\mathrm{X}$ & & & & \\
\hline & Sciaenidae (drums and croakers) & & & & & & & & & & & \\
\hline 357 & Atractoscion nobilis (Ayres, 1860) & White Seabass & & $\mathrm{X}$ & & & & $\mathrm{X}$ & & & & \\
\hline 358 & Genyonemus lineatus (Ayres, 1855) & White Croaker & $\mathrm{X}$ & $\mathrm{X}$ & & & & & & & M & \\
\hline 359 & $\dagger \quad$ Menticirrhus undulatus (Girard, 1854) & California Corbina & & $\mathrm{X}$ & & & $\mathrm{X}$ & $\mathrm{X}$ & & & & \\
\hline 360 & Seriphus politus Ayres, 1860 & Queenfish & $\mathrm{X}$ & $\mathrm{X}$ & & & & & & & $\mathrm{M} / \mathrm{P}$ & \\
\hline & Kyphosidae (sea chubs) & & & & & & & & & & & \\
\hline 361 & Girella nigricans (Ayres, 1860) & Opaleye & $\mathrm{X}$ & $\mathrm{X}$ & & & & & & & & \\
\hline 362 & Hermosilla azurea Jenkins \& Evermann, 1889 & Zebraperch & & $\mathrm{X}$ & $\mathrm{X}$ & & & & & & & \\
\hline 363 & Kyphosus vaigiensis (Quoy \& Gaimard 1825) & Blue-bronze Chub & $\mathrm{X}$ & & & & & $\mathrm{X}$ & & & & \\
\hline 364 & Medialuna californiensis (Steindachner, 1876) & Halfmoon & $\mathrm{X}$ & $\mathrm{X}$ & & & & $\mathrm{X}$ & & & & \\
\hline & Pentacerotidae (armorheads) & & & & & & & & & & & \\
\hline 365 & Pseudopentaceros wheeleri (Hardy, 1983) & $\begin{array}{c}\text { North Pacific } \\
\text { Armorhead }\end{array}$ & $\mathrm{X}$ & & & & & & & & & \\
\hline & Oplegnathidae (knifejaws) & & & & & & & & & & & \\
\hline 366 & Oplegnathus fasciatus (Temminck \& Schlegel, 1844) & Barred Knifejaw & & $\mathrm{X}$ & $\mathrm{X}$ & & & & & & & $\mathrm{X}$ \\
\hline & Embiotocidae (surfperches) & & & & & & & & & & & \\
\hline 367 & Amphistichus argenteus Agassiz, 1854 & Barred Surfperch & $\mathrm{X}$ & & & & & & & & & \\
\hline 368 & Amphistichus koelzi (Hubbs, 1933) & Calico Surfperch & $\mathrm{X}$ & & & & & & & & & \\
\hline 369 & Amphistichus rhodoterus (Agassiz, 1854) & Redtail Surfperch & $\mathrm{X}$ & & & & & & & & & \\
\hline 370 & Brachyistius frenatus Gill, 1862 & Kelp Perch & $\mathrm{X}$ & & & & & & & & & \\
\hline 371 & Cymatogaster aggregata Gibbons, 1854 & Shiner Perch & $\mathrm{X}$ & $\mathrm{X}$ & & & & & & & $\mathrm{M} / \mathrm{P}$ & \\
\hline 372 & Embiotoca caryi Agassiz, 1853 & Rainbow Seaperch & $\mathrm{X}$ & & & & & & & & M & \\
\hline 373 & Embiotoca jacksoni Agassiz, 1853 & Black Perch & $\mathrm{X}$ & $\mathrm{X}$ & & & & & & & $\mathrm{M} / \mathrm{P}$ & \\
\hline 374 & Embiotoca lateralis Agassiz, 1854 & Striped Seaperch & $\mathrm{X}$ & & & & & & & & & \\
\hline 375 & Hyperprosopon argenteum Gibbons, 1854 & Walleye Surfperch & $\mathrm{X}$ & $\mathrm{X}$ & & & & & & & $\mathrm{M} / \mathrm{P}$ & \\
\hline 376 & Hyperprosopon ellipticum (Gibbons, 1854) & Silver Surfperch & $\mathrm{X}$ & & & & & & & & M & \\
\hline 377 & Hypocritichthys analis (Agassiz, 1861) & Spotfin Surfperch & $\mathrm{X}$ & & & & & & & & & \\
\hline 378 & Micrometrus aurora (Jordan \& Gilbert, 1880) & Reef Perch & $\mathrm{X}$ & $\mathrm{X}$ & & $\mathrm{X}$ & & & & & & \\
\hline 379 & Micrometrus minimus (Gibbons, 1854) & Dwarf Perch & $\mathrm{X}$ & $\mathrm{X}$ & & & & & & & $\mathrm{M} / \mathrm{P}$ & \\
\hline 380 & Phanerodon atripes (Jordan \& Gilbert, 1880) & Sharpnose Seaperch & $\mathrm{X}$ & $\mathrm{X}$ & & $\mathrm{X}$ & & & & & & \\
\hline 381 & Phanerodon furcatus Girard, 1854 & White Seaperch & $\mathrm{X}$ & $\mathrm{X}$ & & & & & & & $\mathrm{M} / \mathrm{P}$ & \\
\hline 382 & Phanerodon vacca (Girard, 1855) & Pile Perch & $\mathrm{X}$ & $\mathrm{X}$ & & & & & & & $\mathrm{M} / \mathrm{P}$ & \\
\hline 383 & Rhacochilus toxotes Agassiz, 1854 & Rubberlip Seaperch & $\mathrm{X}$ & $\mathrm{X}$ & & & & & & & $\mathrm{M} / \mathrm{P}$ & \\
\hline 384 & Zalembius rosaceus (Jordan \& Gilbert, 1880) & Pink Seaperch & $\mathrm{X}$ & & & & & & & & & \\
\hline
\end{tabular}




\begin{tabular}{|c|c|c|c|c|c|c|c|c|c|c|c|c|}
\hline & \multirow[b]{2}{*}{ Scientific name } & \multirow[b]{2}{*}{ Common name } & \multicolumn{3}{|c|}{ Basis } & \multicolumn{4}{|c|}{ Ancillary record } & \multicolumn{3}{|c|}{ Occurrence of note } \\
\hline & & & 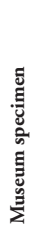 & 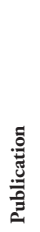 & 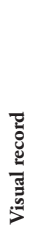 & 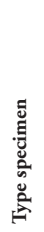 & 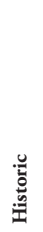 & 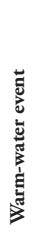 & 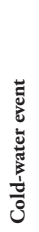 & 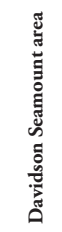 & 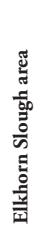 & 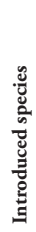 \\
\hline & \multicolumn{12}{|l|}{ Pomacentridae (damselfishes) } \\
\hline 385 & Chromis punctipinnis (Cooper, 1863) & Blacksmith & $\mathrm{X}$ & $\mathrm{X}$ & & & & $\mathrm{X}$ & & & & \\
\hline \multirow[t]{2}{*}{386} & Hypsypops rubicundus (Girard, 1854) & Garibaldi & $\mathrm{X}$ & $\mathrm{X}$ & $\mathrm{X}$ & $\mathrm{X}$ & $\mathrm{X}$ & $\mathrm{X}$ & & & & \\
\hline & bridae (wrasses) & & & & & & & & & & & \\
\hline 387 & Oxyjulis californica Günther, 1861 & Señorita & $\mathrm{X}$ & $\mathrm{X}$ & & $\mathrm{X}$ & & & & & M & \\
\hline \multirow[t]{2}{*}{388} & Semicossyphus pulcher (Ayres, 1854) & California Sheephead & $\mathrm{X}$ & $\mathrm{X}$ & & & & $\mathrm{X}$ & & & & \\
\hline & thymasteridae (ronquils) & & & & & & & & & & & \\
\hline 389 & Rathbunella alleni Gilbert, 1904 & Stripefin Ronquil & $\mathrm{X}$ & $\mathrm{X}$ & & $\mathrm{X}$ & & & & & & \\
\hline \multirow[t]{2}{*}{390} & Ronquilus jordani (Gilbert, 1889) & Northern Ronquil & $\mathrm{X}$ & & & & & & & & & \\
\hline & arcidae (eelpouts) & & & & & & & & & & & \\
\hline 391 & Bothrocara brunneum (Bean, 1890) & Twoline Eelpout & $\mathrm{X}$ & $\mathrm{X}$ & $\mathrm{X}$ & & & & & $\mathrm{P} / \mathrm{V}$ & & \\
\hline 392 & Bothrocara molle Bean, 1890 & Soft Eelpout & $\mathrm{X}$ & & & & & & & & & \\
\hline 393 & Eucryphycus californicus (Starks \& Mann, 1911) & Persimmon Eelpout & $\mathrm{X}$ & $\mathrm{X}$ & & & & & & & & \\
\hline 394 & Lycenchelys callista \&erson, 1995 & eelpout & $\mathrm{X}$ & $\mathrm{X}$ & & $\mathrm{X}$ & & & & & & \\
\hline 395 & Lycenchelys camchatica (Gilbert \& Burke, 1912) & Kamchatka Eelpout & $\mathrm{X}$ & $\mathrm{X}$ & & & & & & & & \\
\hline 396 & Lycenchelys crotalinus (Gilbert, 1890) & Snakehead Eelpout & $\mathrm{X}$ & & & & & & & & & \\
\hline 397 & Lycenchelys jordani (Evermann \& Goldsborough, 1907) & Shortjaw Eelpout & $\mathrm{X}$ & & & & & & & & & \\
\hline 398 & Lycenchelys micropora \&riashev, 1955 & Manytoothed Eelpout & $\mathrm{X}$ & & & & & & & & & \\
\hline 399 & Lycenchelys monstrosa \&erson, 1982 & eelpout & $\mathrm{X}$ & & & & & & & & & \\
\hline 400 & Lycodapus dermatinus Gilbert, 1896 & Looseskin Eelpout & $\mathrm{X}$ & & & & & & & & & \\
\hline 401 & Lycodapus fierasfer Gilbert, 1890 & Blackmouth Eelpout & $\mathrm{X}$ & $\mathrm{X}$ & $\mathrm{X}$ & & & & & $\mathrm{P} / \mathrm{V}$ & & \\
\hline 402 & Lycodapus móribularis Gilbert, 1915 & Pallid Eelpout & $\mathrm{X}$ & $\mathrm{X}$ & $\mathrm{X}$ & $\mathrm{X}$ & & & & $\mathrm{P} / \mathrm{V}$ & & \\
\hline 403 & Lycodapus pachysoma Peden \& \&erson, 1978 & Stout Eelpout & $\mathrm{X}$ & & & & & & & M & & \\
\hline 404 & Lycodapus psarostomatus Peden \& \&erson, 1981 & Specklemouth Eelpout & $\mathrm{X}$ & & & & & & & & & \\
\hline 405 & Lycodes cortezianus (Gilbert, 1890) & Bigfin Eelpout & $\mathrm{X}$ & & & & & & & & & \\
\hline 406 & Lycodes diapterus Gilbert, 1892 & Black Eelpout & $\mathrm{X}$ & & & & & & & & & \\
\hline 407 & Lycodes pacificus (Collett, 1879) & Blackbelly Eelpout & $\mathrm{X}$ & & & & & & & & & \\
\hline 408 & Lyconema barbatum Gilbert, 1896 & Bearded Eelpout & $\mathrm{X}$ & $\mathrm{X}$ & & $\mathrm{X}$ & & & & & & \\
\hline 409 & Melanostigma pammelas Gilbert, 1896 & Midwater Eelpout & $\mathrm{X}$ & $\mathrm{X}$ & & $\mathrm{X}$ & & & & & & \\
\hline 410 & Pachycara bulbiceps (Garman, 1899) & Snubnose Eelpout & & $\mathrm{X}$ & $\mathrm{X}$ & & & & & $\mathrm{P} / \mathrm{V}$ & & \\
\hline \multirow[t]{2}{*}{411} & Pachycara karenae \&erson, 2012 & eelpout & $\mathrm{X}$ & $\mathrm{X}$ & & $\mathrm{X}$ & & & & & & \\
\hline & chaeidae (pricklebacks) & & & & & & & & & & & \\
\hline 412 & Anoplarchus purpurescens Gill, 1861 & High Cockscomb & $\mathrm{X}$ & & & & & & & & & \\
\hline 413 & Cebidichthys violaceus (Girard, 1854) & $\begin{array}{c}\text { Monkeyface Prick- } \\
\text { leback }\end{array}$ & $\mathrm{X}$ & & & & & & & & M & \\
\hline 414 & Chirolophis nugator (Jordan \& Williams, 1895) & Mosshead Warbonnet & $\mathrm{X}$ & & & & & & & & & \\
\hline 415 & Ernogrammus walkeri Follett \& Powell, 1988 & Masked Prickleback & $\mathrm{X}$ & $\mathrm{X}$ & & $\mathrm{X}$ & & & & & & \\
\hline 416 & Esselenichthys carli (Follett \& \&erson, 1990) & Threeline Prickleback & $\mathrm{X}$ & $\mathrm{X}$ & & & & & & & & \\
\hline 417 & Kasatkia seigeli Posner \& Lavenberg, 1999 & Sixspot Prickleback & $\mathrm{X}$ & & & & & & & & & \\
\hline 418 & Phytichthys chirus (Jordan \& Gilbert, 1880) & Ribbon Prickleback & $\mathrm{X}$ & $\mathrm{X}$ & & $\mathrm{X}$ & & & & & & \\
\hline 419 & Plagiogrammus hopkinsii Bean, 1894 & Crisscross Prickleback & $\mathrm{X}$ & $\mathrm{X}$ & & $\mathrm{X}$ & & & & & & \\
\hline 420 & Plectobranchus evides Gilbert, 1890 & Bluebarred Prickleback & $\mathrm{X}$ & $\mathrm{X}$ & & & & & & & & \\
\hline 421 & Xiphister atropurpureus (Kittlitz, 1858) & Black Prickleback & $\mathrm{X}$ & & & & & & & & & \\
\hline \multirow[t]{2}{*}{422} & Xiphister mucosus (Girard, 1858) & Rock Prickleback & $\mathrm{X}$ & & & & & & & & & \\
\hline & olidae (gunnels) & & & & & & & & & & & \\
\hline 423 & Apodichthys flavidus Girard, 1854 & Penpoint Gunnel & $\mathrm{X}$ & & & & & & & & M & \\
\hline 424 & Apodichthys fucorum Jordan \& Gilbert, 1880 & Rockweed Gunnel & $\mathrm{X}$ & $\mathrm{X}$ & & $\mathrm{X}$ & & & & & & \\
\hline 425 & Pholis clemensi Rosenblatt, 1964 & Longfin Gunnel & & $\mathrm{X}$ & $\mathrm{X}$ & & & & & & & \\
\hline 426 & Pholis ornata (Girard, 1854) & Saddleback Gunnel & $\mathrm{X}$ & & & & & & & & & \\
\hline 427 & Pholis schultzi Schultz, 1931 & Red Gunnel & $\mathrm{X}$ & & & & & & & & & \\
\hline 428 & Ulvicola sanctaerosae Gilbert \& Starks, 1897 & Kelp Gunnel & $\mathrm{X}$ & & & & & & & & & \\
\hline
\end{tabular}




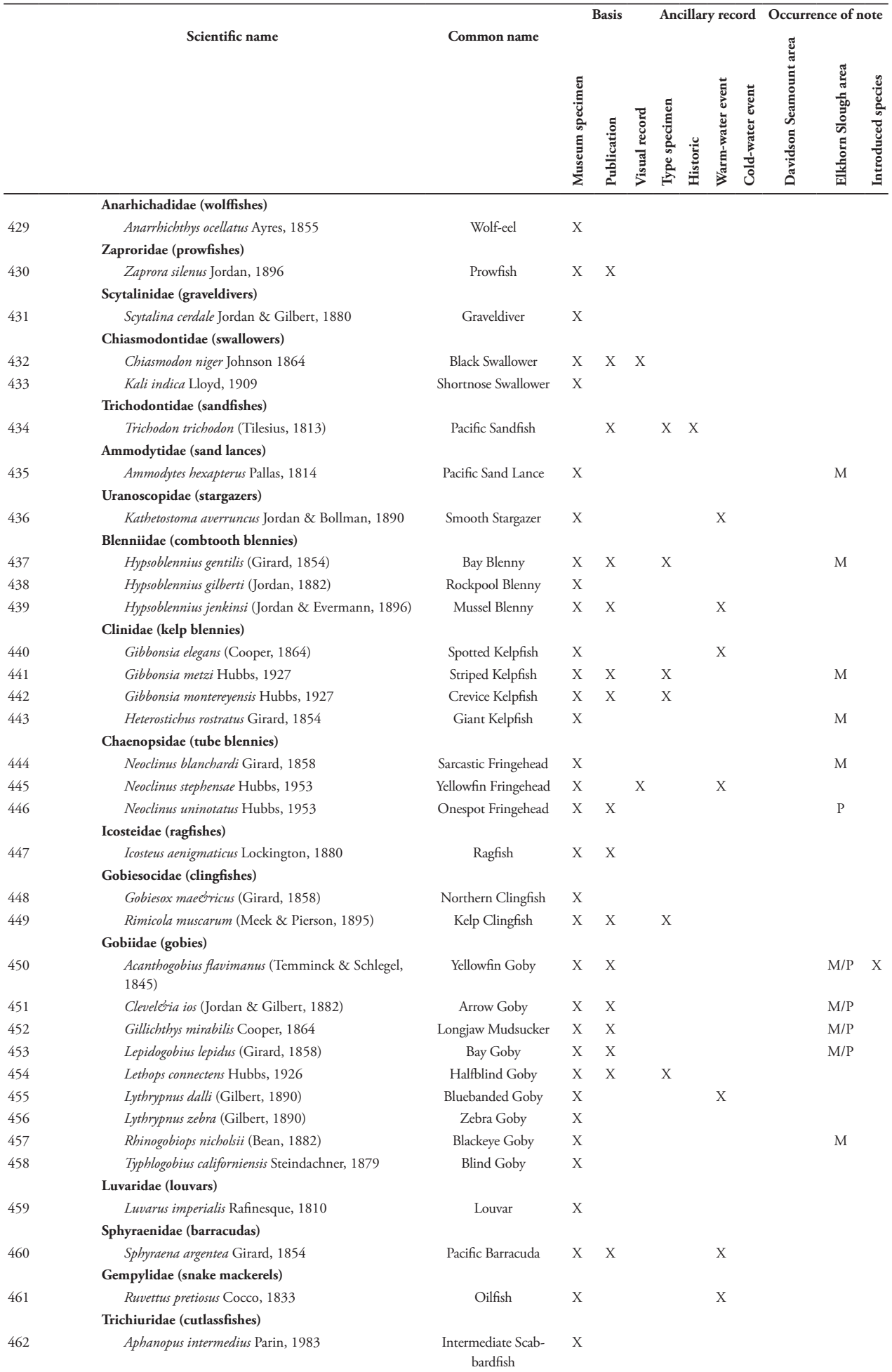




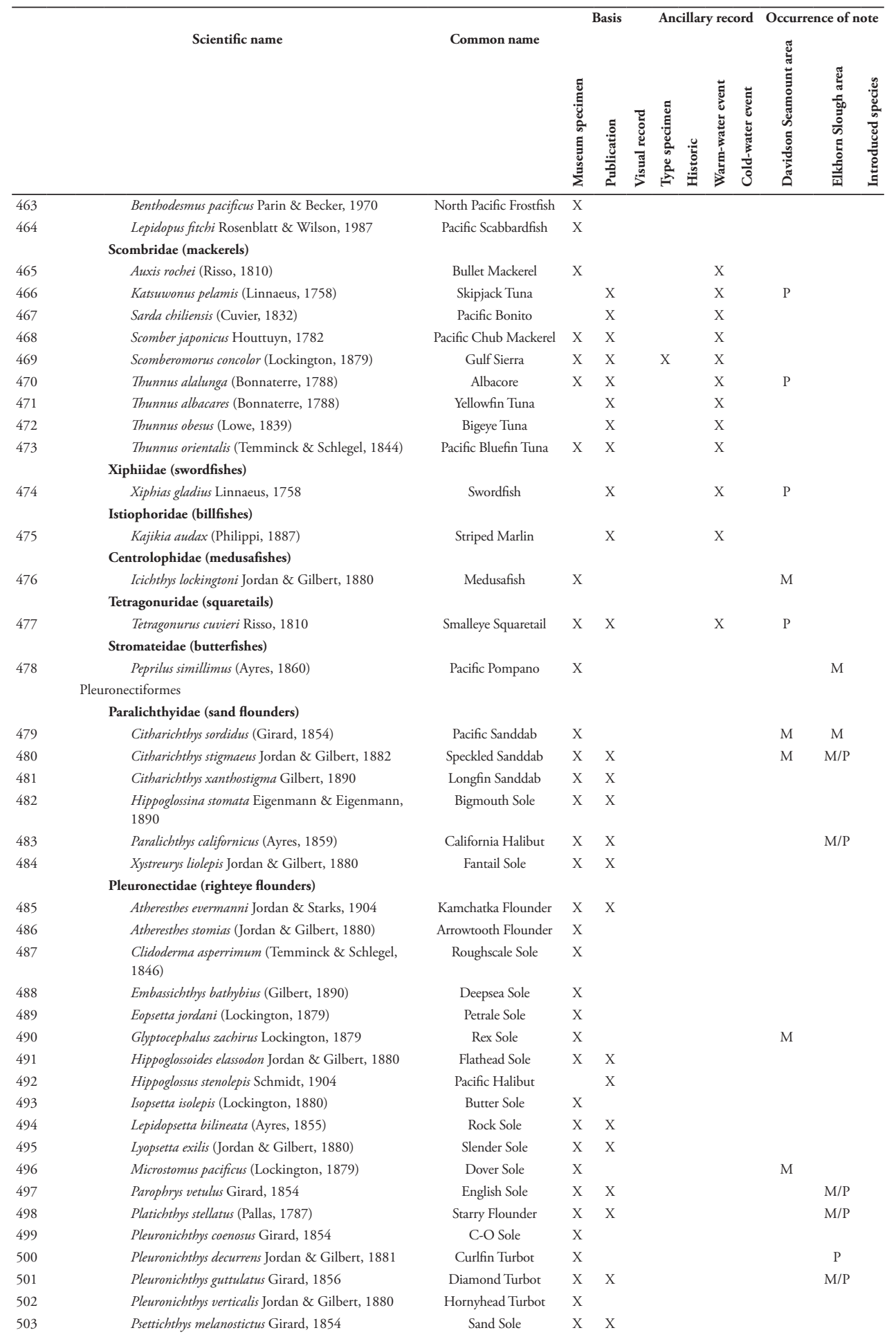




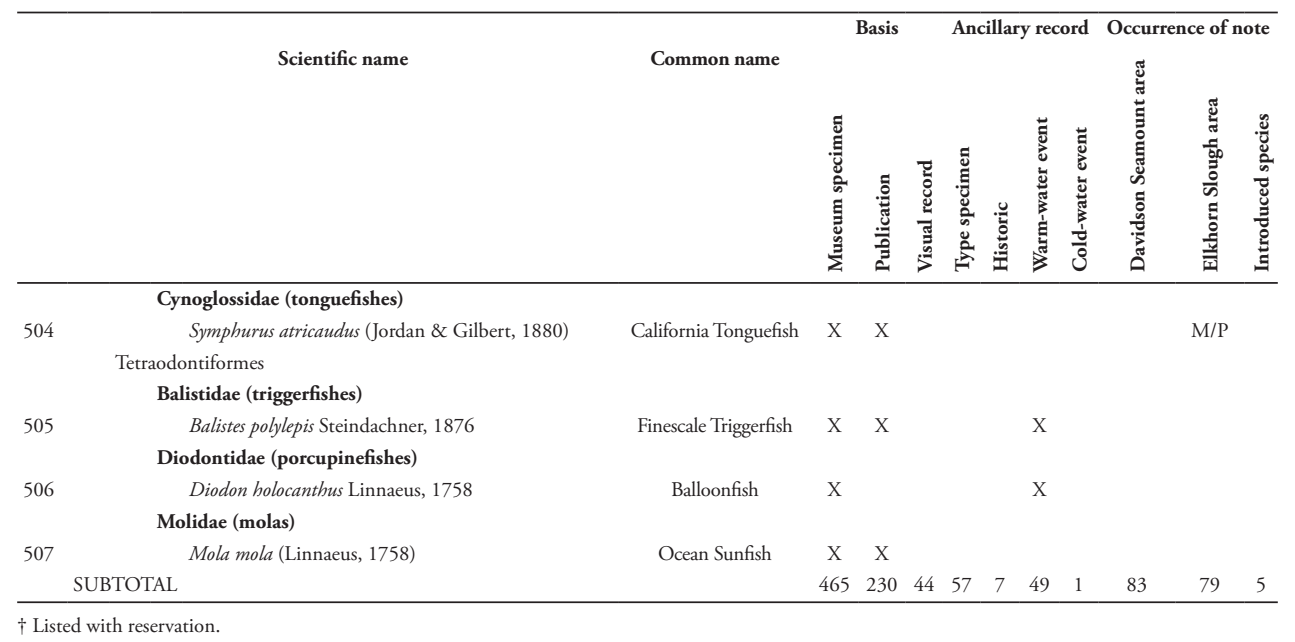

\section{Annotated checklist}

Order Myxiniformes

Family MYXINIDAE - hagfishes

Eptatretus deani (Evermann \& Goldsborough, 1907). Black Hagfish. Museum specimens: CAS-ICH 39879 (west of Yankee Point, WC Ruark); CAS-ICH 42563 (off Cypress Point, RN Lea); MLMLF0003 (Monterey Bay, R Parrish).

Eptatretus stoutii (Lockington, 1878). Pacific Hagfish. Museum specimens: MLMLF0007 (1.5 miles south of Kirby Park at Elkhorn Slough, LT Ackerman); CAS-SU 58418 (Monterey Bay, JC Briggs); CAS-SU 66927 (Monterey Bay, CL Hubbs).

\section{Order Petromyzontiformes}

Family PETROMYZONIDAE - lampreys

Entosphenus tridentatus (Gairdner, 1836). Pacific Lamprey. Museum specimens: MLMLF0010 (Monterey Bay, "on throat of Onchorhynchus tshawytscha", LT Ackerman); MLMLF0011 (Moss Landing beach; Ackerman, Lewis, Lindquist); CAS-SU 11299 (Pacific Grove, identifier unknown).

\section{Order Chimaeriformes \\ Family CHIMAERIDAE - shortnose chimaeras}

Hydrolagus colliei (Lay \& Bennett, 1839). Spotted Ratfish. Described from Monterey. Museum specimens: Type material (original material not preserved); CAS-ICH 
51176 (west of Davenport, L Podshadley); CAS-SU 10829 (Monterey, RL Bolin); CAS-SU 62378 (off Point Sur, RL Bolin). Publication: Lay and Bennett (1839) (original description).

Hydrolagus cf. trolli Didier and Séret, 2002. Pointy-nosed Blue Chimaera. Publications: Lundsten et al. (2009) and Reichert et al. (2016) (Davidson Seamount). Visual records: MBARI T1102-04 (Davidson Seamount, DA Didier and D Ebert; Jacobson Stout et al. 2017); MBARI T1075-02 (Monterey Canyon DA Didier and D Ebert; Jacobson Stout et al. 2017). Naming convention follows Reichert et al. (2016); specimens cannot yet be confirmed as Hydrolagus trolli until morphometric data and/or DNA samples from preserved specimens have been collected and analyzed. Common name follows Didier and Séret (2002).

\section{Order Heterodontiformes}

Family HETERODONTIDAE - horn sharks

Heterodontus francisci (Girard, 1855). Horn Shark. Described from 'Bay of Monterey, Cal.'. Museum specimens: Type material (original material not located); USNM 112106 (Monterey, DS Jordan). Publication: Girard (1855) (original description).

\section{Order Orectolobiformes}

\section{Family RHINCODONTIDAE - whale sharks}

Rhincodon typus Smith, 1828. Whale Shark. Publication: Ebert et al. (2004) (based on visual records, Monterey Bay during 1928-1930, 1929, 1944, 1947, and off Big Sur during 1989). Ebert et al. (2004) state all reported local sightings (where season and year recorded) were during La Nińa years. Categorized as occurring during cold-water events (i.e., La Niña) based on Ebert et al (2004).

\section{Order Lamniformes}

Family ALOPIIDAE - thresher sharks

Alopias vulpinus (Bonnaterre, 1788). Common Thresher Shark. Museum specimens: CAS-ICH 65976 (off Point San Pedro at San Mateo County, WI Follett); MLMLF0024 (off Manressa State Beach, ME Anderson).

\section{Family CETORHINIDAE - basking sharks}

Cetorhinus maximus (Gunnerus, 1765). Basking Shark. Museum specimens: CASICH 2224 (15 miles off Santa Cruz, idenfier unknown); CAS-ICH 26246 (off Salinas 
River mouth, identifier unknown). Publications: Jordan and Gilbert (1880a) (Monterey during 1880); Jordan (1887a) (Santa Cruz County, Monterey Bay during 1880); Jordan (1887b) (Monterey Bay fishery during 1880); Scofield (1917) (Monterey Bay); Starks (1921) (Monterey); Thompson (1921a) (Monterey); Phillips (1948) (Monterey Bay fishery during 1946-1947, with reference to harpooning for sport in Monterey Bay during 1924); Follett (1966) (off Salinas River mouth, CAS-ICH 26246).

\section{Family LAMNIDAE - mackerel sharks}

Carcharodon carcharias (Linnaeus, 1758). White Shark. Museum specimens: CASICH 26245 (off Salinas River mouth, WI Follett); CAS-ICH 26308 (Pebble Beach, Monterey County; WI Follett and LJ Dempster); CAS-ICH 26678 (off La Selva Beach, WI Follett). Publication: Jordan and Gilbert (1880a) ("Point Carmelo, near Monterey").

Isurus oxyrinchus Rafinesque, 1810. Shortfin Mako. Museum specimens: CAS-ICH 53202 (west of Princeton Harbor during 1983, El Niño, identifier unknown); CASICH 55473 (off Santa Cruz, identifier unknown). Categorized as occurring during warm-water events (e.g., El Niño).

Lamna ditropis Hubbs and Follett, 1947. Salmon Shark. Museum specimens: CASICH 26710 (Monterey Bay, WI Follett and LJ Dempster); CAS-SU 12656 (Monterey Bay, LJV Compagno). Publication: Carlisle et al. (2015) (numerous strandings along US west coast; in MBNMS from Montara State Beach to San Simeon area).

\section{Order Carcharhiniformes \\ Family SCYLIORHINIDAE - cat sharks}

Based on molecular data and morphology, Iglesias et al. (2005) proposed resurrection of family Pentanchidae, and assigned the content of Pentanchinae (i.e., including the genera Apristurus and Parmaturus) as in Compagno (1988). This proposed change is not yet widely accepted; therefore, we chose to keep deepwater catsharks in the family Scyliorhinidae.

Apristurus brunneus (Gilbert, 1892). Brown Cat Shark. Museum specimens: CASICH 37479 (Monterey Bay, LJV Compagno); CAS-ICH 37512 (west of Yankee Point, WN Eschmeyer); MLMLF0028 (off Point Sur, ME Anderson).

Apristurus kampae Taylor, 1972. Longnose Cat Shark. Museum specimens: CASICH 58482 (off Point Sur, RN Lea); CAS-ICH 58487 (west of Point Año Nuevo, RN 
Lea); MLMLF0030 (off Cypress Point, ME Anderson). Publication: Anderson et al. (1979) (off Cypress Point, MLMLF0030).

Cephaloscyllium ventriosum (Garman, 1880). Swell Shark. Museum specimens: CAS-ICH 18050 (Monterey, LJV Compagno); CAS-ICH 52983 (Santa Cruz, M Moriguchi).

Parmaturus xaniurus (Gilbert, 1892). Filetail Cat Shark. Museum specimens: CASICH 37499 (west of Point Año Nuevo, T Iwamoto); CAS-ICH 37513 (west of Yankee Point, WN Eschmeyer); MLMLF0036 (Monterey Bay, L Talent).

\section{Family TRIAKIDAE - hound sharks}

Galeorhinus galeus (Linnaeus, 1758). Tope. Museum specimens: CAS-ICH 55475 (Monterey Bay area, WI Follett); MLMLF0040 (Monterey Bay, ME Anderson); CASSU 4148 (Monterey, LJV Compagno).

Mustelus californicus Gill, 1864. Gray Smoothhound. Museum specimens: CASICH 13158 (Monterey Bay, JVL Compagno); CAS-ICH 65045 (Elkhorn Slough, WI Follett); MLMLF0043 (Kirby Park at Elkhorn Slough, LT Ackerman). Publications: Herald (1953) (Elkhorn Slough); Yoklavich et al. (1991) (Kirby Park and Hudson's Landing at Elkhorn Slough).

Mustelus henlei (Gill, 1863). Brown Smoothhound. Museum specimens: CAS-ICH 40629 (Elkhorn Slough, LJV Compagno); MLMLF0046 (Monterey Bay, JP Harville); MLMLF047 (Monterey Bay, L Compagno).

Triakis semifasciata Girard, 1855. Leopard Shark. Museum specimens: CAS-ICH 13162 (Monterey Bay, WM Morton); CAS-ICH 75994 (Kirby Park at Elkhorn Slough, DT Anderson); MLMLF0050 (1.5 mile below Kirby Park at Elkhorn Slough, LT Ackerman). Publications: Herald (1953) (Elkhorn Slough); Herald and Dempster (1952) (Elkhorn Slough); Yoklavich et al. (1991) (Hwy 1 Bridge, Dairy, Kirby Park, and Hudson's Landing at Elkhorn Slough).

\section{Family CARCHARHINIDAE - requiem sharks}

Prionace glauca (Linnaeus, 1758). Blue Shark. Museum specimens: CAS-ICH 65086 (Moss Beach, WI Follett); SIO 62-413 (SW of Pigeon Point, RL McNeely); CAS-SU 53183 (off Santa Cruz, WC Freihofer). Publication: Fitch (1949) (Davidson Seamount). 


\section{Order Hexanchiformes \\ Family HEXANCHIDAE - cow sharks}

Hexanchus griseus (Bonnaterre, 1788). Bluntnose Sixgill Shark. Museum specimens: MLMLF0014 (off Davenport, LT Ackerman); CAS-SU 13291 (Monterey Bay, EC Starks); CAS-SU 49011 (Monterey Bay area, WI Follett).

Notorynchus cepedianus (Péron, 1807). Broadnose Sevengill Shark. Museum specimen: CAS-SU 40909 ("Monterey Bay area of California?", WI Follett). In addition, 15 collections from San Francisco Bay are accessioned at California Academy of Sciences; which presumably traversed MBNMS waters.

\section{Order Squaliformes}

Family ECHINORHINIDAE - bramble sharks

Echinorhinus cookei Pietschmann, 1928. Prickly Shark. Museum specimens: MLMLF0068 (Monterey Canyon, D Lewis); UF 41736 (off Moss Landing, D Ebert).

\section{Family SQUALIDAE - dogfish sharks}

Squalus suckleyi (Girard, 1855). Pacific Spiny Dogfish. Museum specimens: MLMLF0064 (off Santa Cruz, GV Morejohn); MLMLF0065 (Monterey Bay, ME Anderson); CAS-SU 58376 (Monterey Bay, JC Briggs).

\section{Family SOMNIOSIDAE - sleeper sharks}

Somniosus pacificus Bigelow and Schroeder, 1944. Pacific Sleeper Shark. Museum specimens: CAS-ICH 53524 (off Pescadero Point, T Iwamoto); MLMLF0061 (Monterey Bay, ME Anderson); MLMLF0062 (Monterey Bay, GV Morejohn). Publication: Anderson et al. (1979) (Monterey Bay, MLMLF0062).

\section{Order Squatiniformes}

Family SQUATINIDAE - angel sharks

Squatina californica Ayres, 1859. Pacific Angel Shark. Museum specimens: CAS-ICH 65090 (Monterey Bay, identifier unknown); MLMLF0069 (Monterey Bay, D Lewis). 


\section{Order Torpediniformes \\ Family TORPEDINIDAE - torpedo electric rays}

Tetronarce californica (Ayres, 1855). Pacific Electric Ray. Museum specimens: CASICH 37472 (Monterey Bay, T Iwamoto); CAS-SU 21416 (Monterey Bay, LJV Compagno); CAS-SU 58396 (Monterey Bay, JC Briggs). Previously recognized as Torpedo californica. We follow Carvalho et al. (2016), Weigmann (2016), and current usage in Catalog of Fishes (Eschmeyer et al. 2017); with placement in Tetronarce.

\section{Order Rajiformes \\ Family RHINOBATIDAE - guitarfishes}

Pseudobatos productus (Ayres, 1854). Shovelnose Guitarfish. Described from Monterey. Museum specimens: Syntype (USNM 1009); CAS-ICH 65978 (Moss Landing at Elkhorn Slough, WI Follett); MLMLF0079 (Kirby Park at Elkhorn Slough, LT Ackerman). Publications: Ayres (1854) (original description); Herald and Dempster (1952) (Elkhorn Slough); Herald (1953) (Elkhorn Slough). Previously recognized as Rhinobatos productus. We follow Last et al. (2016a), Séret et al. (2016), and current usage in Catalog of Fishes (Eschmeyer et al. 2017); with placement in Pseudobatos.

\section{Family RAJIDAE - skates}

Amblyraja badia (Garman, 1899). Broad Skate. Museum specimen: CAS-ICH 58604 (Point Sur, M Stehmann and RN Lea). Publication: Burton and Lundsten (2008) (Davidson Seamount, based on imagery). Visual record: MBARI/NOAA D943-06 (Davidson Seamount, D Ebert and L Kuhnz; Jacobson Stout et al. 2017). Last et al. (2016c) suggest $A$. badia may be junior synonym of $A$. hyperborea. Proposed change is unresolved and not widely accepted; we do not accept revision at this time. Common name follows Ebert (2003).

Beringraja binoculata (Girard, 1855). Big Skate. Museum specimens: CAS-ICH 12875 (off Point Montara Light, EC Johnston); CAS-ICH 40328 (5.5. miles SSW of Point Piedras Blancas, WC Ruark); CAS-ICH 47407 (west of Pajaro River mouth, L Ayres); MLMLF0104 (Monterey Bay, E Osada).

Beringraja inornata (Jordan \& Gilbert, 1881). California Skate. Museum specimens: CAS-ICH 232351 (west of Santa Cruz, JD Hopkirk); CAS-SU 4032 (Monterey, DS Jordan); CAS-SU 23988 (Pacific Grove, EC Starks). Previously recognized as Raja inornata. We follow Last et al. (2016c), and current usage in Catalog of Fishes 
(Eschmeyer et al. 2017); with placement in Beringraja. Raja not found within eastern Pacific.

Beringraja rhina (Jordan \& Gilbert, 1880). Longnose Skate. Described from Monterey Bay and San Francisco Bay. Museum specimens: Syntypes (3 specimens, accession location unknown); CAS-ICH 39891 (Monterey, WC Ruark); CAS-SU 61651 (Monterey Bay, JC Briggs). Publication: Jordan and Gilbert (1880h) (original description). Previously recognized as Raja rhina. We follow Last et al. (2016c), and current usage in Catalog of Fishes (Eschmeyer et al. 2017); with placement in Beringraja. Raja not found within eastern Pacific.

Beringraja stellulata (Jordan \& Gilbert, 1880). Starry Skate. Described from Monterey Bay. Museum specimens: Paratypes [ANSP 414 (1), MNHN A-3295 (1), USNM 26975 (orig. 8, now 2), ZMB 11687 (1), ZMUB 877 (1)]; CASICH 26919 (Pacific Grove, R Ishiyama); CAS-ICH 224344 (off Davenport, D Ebert and C Davis); CAS-SU 13288 (Santa Cruz, LJV Campangno). Publication: Jordan and Gilbert (1880d) (original description). Previously recognized as Raja stellulata. We follow Last et al. (2016c), and current usage in Catalog of Fishes (Eschmeyer et al. 2017); with placement in Beringraja. Raja not found within eastern Pacific.

\section{Family ARHYNCHOBATIDAE - softnose skates}

Bathyraja previously recognized in family Rajidae. We follow Last et al. (2016b), Last et al. (2016c), and current usage in Catalog of Fishes (Eschmeyer et al. 2017); with placement in family Arhynchobatidae.

Bathyraja abyssicola (Gilbert, 1896). Deepsea Skate. Museum specimens: CASICH 38013 (west of Yankee Point, WN Eschmeyer); CAS-ICH 58481 (off Point Sur, RN Lea). Publication: Burton and Lundsten (2008) (Davidson Seamount, based on imagery). Visual records: MBARI/NOAA T427-07 and MBARI/NOAA T427-09 (Davidson Seamount, D Ebert and L Kuhnz).

Bathyraja aleutica (Gilbert, 1896). Aleutian Skate. Museum specimen: CAS-ICH 243646 (Año Nuevo Canyon, identifier unknown; RN Lea examined specimen, radiograph, and confirmed presence of scapular spines).

Bathyraja kincaidii (Garman, 1908). Sandpaper Skate. Museum specimens: CASICH 39852 (off Hurricane Point, RN Lea); CAS-ICH 58485 (west of Point Año Nuevo, RN Lea, previously Bathyraja interrupta); CAS-SU 5434 (near La Cruz Canyon, ME Anderson, previously B. interrupta). Previously recognized as B. interupta. 
We follow Last et al. (2016b), with placement in Bathyraja. California records previously identified as $B$. interrupta now deemed to be $B$. kincaidii; $B$. interrupta not known south of Bering Sea.

Bathyraja trachura (Gilbert, 1892). Roughtail Skate. Museum specimens: CASICH 47392 (Monterey Canyon, RN Lea); CAS-ICH 56106 (off Point Sur, ME Anderson); CAS-ICH 58486 (west of Point Año Nuevo, RN Lea). Common name follows Ebert (2003).

\section{Family PLATYRHINIDAE - thornbacks}

Platyrhinoidis triseriata (Jordan \& Gilbert, 1880). Thornback. Museum specimens: CAS-ICH 20586 and CAS-ICH 63204 (Elkhorn Slough, ES Herald); MLMLF0074 (Elkhorn Slough, A King). Publication: Herald (1953) (Elkhorn Slough). Ordinal classification unresolved at present; here, placed in Order Rajiformes.

\section{Order Myliobatiformes \\ Family UROTRYGONIDAE - round stingrays}

Urobatis halleri (Cooper, 1863). Round Stingray. Museum specimens: CAS-ICH 20587 and CAS-ICH 31371 (Elkhorn Slough, ES Herald); MLMLF0116 (1.5 miles below Kirby Park at Elkhorn Slough, LT Ackerman). Publications: Herald (1953) (Elkhorn Slough); Yoklavich et al. (1991) (Dairy and Kirby Park at Elkhorn Slough).

\section{Family DASYATIDAE - whiptail stingrays}

Pteroplatytrygon violacea (Bonaparte, 1832). Pelagic Stingray. Publication: Mollet (2002) (bycatch in California drift gill net fishery for swordfish within Davidson Seamount Management Zone).

\section{Family MYLIOBATIDAE - eagle rays}

Myliobatis californica Gill, 1865. Bat Ray. Museum specimens: MLMLF0113 (Kirby Park at Elkhorn Slough, E Yarberry); CAS-SU 47326 (Elkhorn Slough, RL Bolin). Publications: Herald (1953) (Elkhorn Slough); Herald and Dempster (1952) (Elkhorn Slough); Yoklavich et al. (1991) (Hwy 1 Bridge, Dairy, Kirby Park, and Hudson's Landing at Elkhorn Slough). 


\section{Order Acipenseriformes \\ Family ACIPENSERIDAE - sturgeons}

Acipenser medirostris Ayres, 1854. Green Sturgeon. Museum specimens: CAS-ICH 53083 (Santa Cruz Pier, D Rawls); CAS-SU 55089 (Moss Landing Beach, WC Freihofer and HH DeWitt). Anadromous.

Acipenser transmontanus Richardson, 1836. White Sturgeon. Museum specimen: CAS-ICH 82295 (Pajaro River Lagoon, RN Lea). Although museum specimens were not collected within MBNMS, this is an anadromous species that enters the ocean (adjacent MBNMS) during its life cycle.

\section{Order Albuliformes} Family ALBULIDAE - bonefishes

Albula gilberti Pfeiler and van der Heiden, 2011. Cortez Bonefish. Museum specimens (under old name Albula vulpes): CAS-SU 13034 (Monterey Bay, identifier unknown); CAS-SU 35301 (Monterey during Mar 1941, El Niño, RL Bolin).

\section{Order Notacanthiformes}

\section{Family HALOSAURIDAE - halosaurs}

Aldrovandia cf. oleosa Sulak 1977. halosaur. Publications: Burton and Lundsten (2008) and Lundsten et al. (2009) (Davidson Seamount, based on imagery, as Aldrovandia sp.). Visual record: MBARI/NOAA T427-11 (Davidson Seamount, GM Cailliet and RH Rosenblatt; Jacobson Stout et al. 2017, as Aldrovandia sp.). Specimen observed at Davidson Seamount (video) confirmed to genus by GM Cailliet (MLML) and R Rosenblatt (SIO); unable to identify to species level. However, Kamikawa and Stevenson (2010) state A. oleosa is the only halosaur and known species of Aldrovandia from the eastern North Pacific. No official common name.

\section{Family NOTACANTHIDAE - deep-sea spiny eels}

Notacanthus chemnitzii Bloch, 1788. Snubnosed Spiny Eel. Museum specimens: CAS-ICH 58441 (Monterey Canyon, RN Lea); SIO 85-52 (off Point Sur, W Wakefield); SIO 88-99 (south of Point Sur, identifier unknown, NMFS). Publication: Lea and Rosenblatt (1987) (Monterey Canyon, CAS-ICH 58441; off Point Sur, SIO $85-52)$. 


\section{Order Anguilliformes \\ Family SYNAPHOBRANCHIDAE - cutthroat eels}

SYNAPHOBRANCHIDAE sp. 1. cutthroat eel. Publications: Burton and Lundsten (2008), and Lundsten et al. (2009) (Davidson Seamount, based on imagery). Visual record: MBARI/NOAA T945-04 (Davidson Seamount, EJ Burton, RN Lea, and L Lundsten; Jacobson Stout et al. 2017). Specimen observed at Davidson Seamount (video) was identified to the family level by EJ Burton (MBNMS), RN Lea (CAS), and L Lundsten (MBARI); Burton and Lundsten (2008). This is the only known record of Synaphobranchidae in MBNMS and may represent a new species (Lundsten et al. 2009). No official common name.

\section{Family OPHICHTHIDAE - snake eels}

Ophichthus triserialis (Kaup, 1856). Pacific Snake Eel. Museum specimen: MLMLF0139 (Moss Landing Harbor during Nov 1967, R Parrish; re-examined by RN Lea, 2017; $1002 \mathrm{~mm}$ ). Miller and Lea (1972) consider rare from California. This species has also been taken from San Francisco Bay (Hopkirk 1965) and WSW of the Klamath River (Quirollo and Dinnel 1975).

Ophichthus zophochir Jordan and Gilbert, 1882. Yellow Snake Eel. Museum specimen: MLMLF0140 (powerplant intake screen at Moss Landing Harbor during Apr 1969, D Varoujean). This species is rather common in southern California waters (Hopkirk 1965). Other specimens were collected from San Francisco Bay in 1964 (CAS-ICH 23683, Hopkirk 1965) and 1999 (leptocephalus stage, Lea and Rosenblatt 2000). Specimens were most likely transported northward, through the MBNMS area, during a warm-water event (Lea and Rosenblatt 2000). This species has also been taken from Humboldt Bay during Jan 1971 (Quirollo and Dinnel 1975).

\section{Family NEMICHTHYIDAE - snipe eels}

Avocettina infans (Günther, 1878). Blackline Snipe Eel. Museum specimen: CASICH 56233 (Point Sur, ME Anderson).

Nemichthys scolopaceus Richardson, 1848. Slender Snipe Eel. Museum specimen: CAS-ICH 216851 (off Point Sur, RN Lea).

\section{Family NETTASTOMATIDAE - duckbill eels}

Facciolella equatorialis (Gilbert, 1891). Dogface Witch Eel. Visual records: CSUMB Dive 75-T1 (Carmel Canyon during Jun 2010, -345 m, 2 specimens, reviewed by RN 
Lea; SIMoN (2018) (IfAME/MBNMS/MARE/TNC). Observation occurred during warm-water event (e.g., El Niño).

Venefica tentaculata Garman, 1899. Longnose Witch Eel. Publication: Burton and Lundsten (2008) (Davidson Seamount, based on imagery). Visual record: MBARI/ NOAA T427-11 (Davidson Seamount, GM Cailliet, RN Lea, and RH Rosenblatt).

\section{Family SERRIVOMERIDAE - sawtooth eels}

Serrivomer sector Garman, 1899. Sawtooth Eel. Museum specimen: SIO 67-112 (Monterey Canyon, CL Hubbs). Publication: Burton and Lundsten (2008) (Davidson Seamount, based on imagery). Visual record: MBARI/NOAA T944-10 (Davidson Seamount, Jacobson Stout et al. 2017).

\section{Order Saccopharyngiformes}

Family CYEMATIDAE - bobtail eels

Cyema atrum Günther, 1878. Bobtail Eel. Museum specimen: CAS-ICH 63700 (Monterey Canyon, R Bolin). Publication: Burton and Lundsten (2008) (Davidson Seamount, based on imagery). Visual record: MBARI/NOAA T425-11 (Davidson Seamount, EJ Burton and T Trejo).

\section{Family SACCOPHARYNGIDAE - whiptail gulpers}

Saccopharynx lavenbergi Nielsen and Bertelsen, 1985. Whiptail Gulper. Visual records: MBARI T622-07 and MBARI V2869-05 (Monterey Bay, Jacobson Stout et al. 2017).

\section{Family EURYPHARYNGIDAE - gulpers}

Eurypharynx pelecanoides Vaillant, 1882. Umbrellamouth Gulper.Visual record: MBARI D198-02 (Monterey Canyon, Jacobson Stout et al. 2017).

\section{Order Clupeiformes}

Family ENGRAULIDAE - anchovies

Engraulis mordax Girard, 1854. Northern Anchovy. Museum specimens: CAS-ICH 65975 (off Point San Pedro, WI Follett); CAS-ICH 211630 (Monterey Canyon, RL Bolin); CAS-ICH 232634 (Elkhorn Slough, DW Behrens); SWFSC uncatalogued 
(Davidson Seamount during May 2015, W Watson). Publication: Yoklavich et al. (1991) (Hwy 1 Bridge, Dairy, Kirby Park, and Hudson's Landing at Elkhorn Slough).

\section{Family CLUPEIDAE - herrings}

Alosa sapidissima (Wilson, 1811). American Shad. Museum specimens: KU 23736 (west of Point Año Nuevo, M Domeier); MLMLF0146 (Elkhorn Slough near railroad bridge, LT Ackerman); SIO 05-88 (west of Lucia, D Kamikawa). Introduced from the Atlantic; anadromous (Miller and Lea 1972).

Clupea pallasii Valenciennes, 1847. Pacific Herring. Museum specimens: CAS-ICH 25755 (Santa Cruz Municipal Wharf, WI Follett); CAS-SU 58410 (Elkhorn Slough, JC Briggs); CAS-SU 60786 (Monterey Bay, RL Bolin). Publication: Yoklavich et al. (1991) (Dairy, Kirby Park, and Hudson's Landing at Elkhorn Slough).

Dorosoma petenense (Günther, 1867). Threadfin Shad. Publication: Yoklavich et al. (1991) (Kirby Park and Hudson's Landing at Elkhorn Slough). Findings presented by Yoklavich et al. (1991) are based on otter trawl studies by Barry (1983) and Cailliet et al. (1977). Native to southeastern U.S., and along eastern coast of Mexico and Central America; introduced into California freshwater lakes (Miller and Lea 1972, Dill and Cordone 1997). Live mainly in fresh water and become progressively less abundant as salinity increases; however, can survive and grow in seawater (Moyle 2002).

Etrumeus acuminatus Gilbert, 1890. Round Herring. Museum specimens: CASICH 59626 (off Monterey breakwater at Monterey Bay, RN Lea); CAS-SU 14165 (Monterey Bay, JE Randall). Publication: Phillips (1951b) (one mile offshore between Cape San Martin and Piedras Blancas). Previously recognized as Etrumeus teres (now known to be restricted to eastern North Atlantic). We follow naming convention of Randall and DiBattista (2012), and current usage in Catalog of Fishes (Eschmeyer et al. 2017).

Sardinops sagax (Jenyns, 1842). Pacific Sardine. Museum specimens: CAS-ICH 54931 (Monterey Bay, RN Lea); CAS-SU 58378 (Elkhorn Slough, JC Briggs); SWFSC uncatalogued (Davidson Seamount during May 2015, W Watson).

\section{Order Argentiniformes \\ Family ARGENTINIDAE - argentines}

Argentina sialis Gilbert, 1890. Pacific Argentine. Museum specimens: MLMLF0167 and MLMLF0168 (Monterey Bay, ME Anderson); CAS-SU 35311 (Monterey Bay, RL Bolin); CAS-SU 49737 (off Point Pinos, J Phillips). 


\section{Family MICROSTOMATIDAE - pencilsmelts}

Microstoma sp. (Pacific species). pencilsmelt. Museum specimens: CAS-ICH 239292 (Davidson Seamount, RN Lea and EJ Burton); SIO 67-102 (within Davidson Seamount Management Zone, CL Hubbs); CAS-SU 49800 (Monterey Canyon, TN Fast). Recognized by some researchers as Microstoma microstoma. We follow Ahlstrom et al. (1984), and Moser and Butler (1996), where North Pacific is represented by a single undescribed species (Microstoma sp.). No official common name.

Nansenia candida Cohen, 1958. Bluethroat Argentine. Museum specimen: LACM36264.001 (west of Monterey, identifier unknown).

\section{Family BATHYLAGIDAE - deep-sea smelts}

Four genera previously recognized in family Microstomatidae include: Bathylagoides, Leuroglossus, Lipolagus, and Pseudobathylagus. We follow Nelson et al. (2004), Nelson et al. (2016), and current usage in Catalog of Fishes (Eschmeyer et al. 2017), with placement in family Bathylagidae.

Bathylagoides wesethi (Bolin, 1938). Snubnose Blacksmelt. Described from Monterey Bay. Museum specimens: Holotype (CAS-SU 32971); Paratypes (CAS-SU 32972, CAS-SU 32973); CAS-ICH 239291 (Davidson Seamount, RN Lea and EJ Burton); SIO 67-102 (within Davidson Seamount Management Zone, CL Hubbs). Publications: Bolin (1938) (original description).

Bathylagus pacificus Gilbert, 1890. Pacific Blacksmelt. Museum specimens: CASICH 54824 (Monterey Canyon, ME Anderson); CAS-ICH 55061 (off Point Sur, ME Anderson); CAS-ICH 98332 (Monterey Canyon, RL Bolin).

Leuroglossus stilbius Gilbert, 1890. California Smoothtongue. Museum specimens: CAS-ICH 239320 and CAS-ICH 239348 (Davidson Seamount, RN Lea and EJ Burton); SIO 67-102 (within Davidson Seamount Management Zone, CL Hubbs).

Lipolagus ochotensis (Schmidt, 1938). Popeye Blacksmelt. Museum specimens: CAS-ICH 239374 (off Monterey, RN Lea and EJ Burton); SIO 11-250 (west of Lucia, P Davison); SIO 85-57 (Monterey Canyon, W Wakefield); SWFSC uncatalogued (Davidson Seamount during May 2015, W Watson).

Pseudobathylagus milleri (Jordan \& Gilbert, 1898). Robust Blacksmelt. Museum specimens: SIO 67-102 (within Davidson Seamount Management Zone, CL Hubbs); SWFSC uncatalogued (Davidson Seamount during May 2015, W Watson). 


\section{Family OPISTHOPROCTIDAE - spookfishes}

Bathylychnops exilis Cohen, 1958. Javelin Spookfish. Museum specimen: CAS-ICH 74351 (Monterey Bay, F Tsuji).

Dolichopteryx longipes (Vaillant, 1888). Brownsnout Spookfish. Museum specimen: UW 113807 (NE of Cabrillo Canyon, D Kamikawa).

Macropinna microstoma Chapman, 1939. Barreleye. Museum specimens: CAS-SU 48781 (Monterey Canyon, TN Fast); MLMLF0200 (Monterey Canyon, S Zeiner); SWFSC uncatalogued (Davidson Seamount during May 2015, W Watson). Publications: Bradbury and Cohen (1958) (Monterey Bay); Robison and Reisenbichler (2008) (Monterey Bay and Davidson Seamount). Visual records: MBARI V2571-05 (Monterey Bay, Robison and Reisenbichler 2008, Jacobson Stout et al. 2017); MBARI T098702 (Davidson Seamount, Robison and Reisenbichler 2008, Jacobson Stout et al. 2017).

\section{Family ALEPOCEPHALIDAE - slickheads}

Alepocephalus tenebrosus Gilbert, 1892. California Slickhead. Museum specimens: CAS-ICH 42921 (Partington Canyon, B Antrim); MLMLF0202 (Monterey Bay, RH Parrish); MLMLF0204 (Ascension Canyon, ME Anderson); CAS-SU 65825 (Monterey Canyon, RL Bolin and TN Fast).

Talismania bifurcata (Parr, 1951). Threadfin Slickhead. Museum specimens: CASICH 36776 (Monterey Bay, ME Anderson); CAS-ICH 59661 (Monterey Bay, RN Lea).

\section{Family PLATYTROCTIDAE - tubeshoulders}

Holtbyrnia latifrons Sazonov, 1976. Streaklight Tubeshoulder. Museum specimens: MLMLF0211 (Monterey Bay, ME Anderson); SIO 67-112 (Monterey Canyon, CL Hubbs).

Mentodus eubranchus (Matsui \& Rosenblatt, 1987). tubeshoulder. Museum specimen: CAS-ICH 239349 (Davidson Seamount, RN Lea and EJ Burton). This is the first known record of occurrence within MBNMS (unpublished data, Burton, Lea, and DeVogelaere). No official common name.

Sagamichthys abei Parr, 1953. Shining Tubeshoulder. Museum specimens: CASICH 73324 (Monterey Bay, F Tsuji); MLMLF0217 (Monterey Bay, ME Anderson); SIO 87-16 (off Point Sur, R Snodgrass); SWFSC uncatalogued (Davidson Seamount during May 2015, RN Lea, EJ Burton, W Watson). 


\section{Order Osmeriformes \\ Family OSMERIDAE - smelts}

Allosmerus elongatus (Ayres, 1854). Whitebait Smelt. Museum specimens: CASICH 46529 Half Moon Bay, M Hearne); CAS-SU 58426 (Monterey Bay, JC Briggs); USNM 86558 (Monterey, DS Jordan).

Hypomesus pretiosus (Girard, 1854). Surf Smelt. Museum specimens: CAS-ICH 25756 (Santa Cruz Municipal Wharf, WI Follett); CAS-SU 58391 (Elkhorn Slough, JC Briggs).

Spirinchus starksi (Fisk, 1913). Night Smelt. Museum specimens: CAS-ICH 25470 (San Simeon, CL Hubbs, LC Hubbs, WI Follett et al.); CAS-ICH 212344 (off Point Montara, RL Bolin); MLMLF0221 (Moss Landing Harbor, GE Kukowski); CAS-SU 60470 (Monterey Bay, RL Bolin). Publication: Yoklavich et al. (1991) (Hwy 1 Bridge at Elkhorn Slough).

Spirinchus thaleichthys (Ayres, 1860). Longfin Smelt. Museum specimens: ANSP 7726 (Monterey, HW Fowler); CAS-SU 5215 (SE of Santa Cruz, US Fish Commission).

Thaleichthys pacificus (Richardson, 1837). Eulachon. Museum specimens: CAS-ICH 37473 (Monterey Bay, T Iwamoto); CAS-ICH 54942 (Monterey Bay, ME Anderson); MLMLF0226 (off Davenport at Monterey Bay, ME Anderson).

\section{Order Salmoniformes}

\section{Family SALMONIDAE - trouts and salmons}

Oncorbynchus gorbuscha (Walbaum, 1792). Pink Salmon. Categorized as Historic. Scofield (1916) reported the following: several O. gorbuscha were collected from San Lorenzo River (Santa Cruz Co) in 1916; appears in San Lorenzo River only occasionally; is far out of natural range; strays have been taken in the Sacramento River. More recently, Moyle (2002) stated O. gorbuscha today are considered extremely rare in California and must be regarded as extirpated from the state.

Oncorhynchus keta (Walbaum, 1792). Chum Salmon. Categorized as Historic. Scofield (1916) reported the following: three O. keta were collected from San Lorenzo River (Santa Cruz Co) in 1916; and have been reported from the Sacramento River. More recently, Moyle (2002) stated small runs of $O$. keta were historically present in streams from the Sacramento River north; a few fish are still taken in the Sacramento drainage, but no spawning has been recorded in recent decades; it has become increasingly rare in California, probably always uncommon; no recent records of chum salmon at northern Sacramento River drainage or San Joaquin drainage; considered endangered in California; and population in Sacramento River is extirpated. 
Oncorhynchus kisutch (Walbaum, 1792). Coho Salmon. Museum specimens: CASICH 20840 (Scotts Creek, 1934, AC Jones); CAS-ICH 21044 and CAS-ICH 21048 (San Lorenzo River, 1955, AC Jones); CAS-ICH 210251 (Gazos Creek Lagoon, San Mateo County, 1970, WI Follett); CAS-ICH 210273 (Tunitas Creek, San Mateo County, 1939, JD Hopkirk); CAS-SU 4667 (Waddell Creek, 1895, Rutter and Scofield). Publication: Moyle (2002) [Central California coast (Scott and Waddell Creeks, Santa Cruz County)]. Although museum specimens were not collected within MBNMS, this is an anadromous species that must enter the ocean (adjacent MBNMS) during its life cycle. Central California Coast Coho Salmon were listed as threatened in 1996, and in 2005 reclassified as endangered (NOAA Fisheries 2016).

Oncorhynchus mykiss (Walbaum, 1792). Rainbow Trout (Steelhead). Museum specimens: CAS-ICH 23343 (Santa Cruz, RF Elwell); CAS-SU 15095 (Big Sur at Pfeiffer State Park, C Hubbs); USNM 27356 (Monterey, DS Jordan). Publication: Moyle (2002) [Central California coast steelhead (Aptos Creek); South/Central coast steelhead (Monterey Bay tributaries: Pajaro, Salinas, and Carmel Rivers; small streams of Big Sur coast; intermittent stream of San Luis Obispo)].

Oncorhynchus tshawytscha (Walbaum, 1792). Chinook Salmon. Museum specimens: CAS-ICH 225433 (Monterey Bay, DA Neely); MLMLF0230 (Monterey Bay, E Yarberry); CAS-SU 65851 (off Seaside, JB Phillips). Publications: Gilbert (1896) (Monterey Bay); Moyle (2002) [Central Valley fall-run (Sacramento and San Joaquin Rivers and tributaries); Central Valley spring-run (Sacramento River drainage); Sacramento River winter-run (mainstream Sacramento River)]. Important in recreational and commercial fisheries off central California coast.

\section{Order Stomiiformes}

Family GONOSTOMATIDAE - bristlemouths

Cyclothone acclinidens Garman, 1899. Benttooth Bristlemouth. Museum specimens: CAS-ICH 239350 (Davidson Seamount, RN Lea and EJ Burton); MLMLF0231 and MLMLF0232 (Monterey Bay, ME Anderson); SIO 67-102 (within Davidson Seamount Management Zone, CL Hubbs).

Cyclothone atraria Gilbert, 1905. Black Bristlemouth. Museum specimens: CASICH 239321 and CAS-ICH 239351 (Davidson Seamount, RN Lea and EJ Burton); SIO 67-102 (within Davidson Seamount Management Zone, CL Hubbs).

Cyclothone pallida Brauer, 1902. Tan Bristlemouth. Museum specimens: CAS-ICH 36750 (Monterey Bay, ME Anderson); CAS-ICH 97657 (Monterey Canyon, RL Bolin); CAS-ICH 97660 (off Point Pinos, RL Bolin). 
Cyclothone pseudopallida Mukhacheva, 1964. Slender Bristlemouth. Museum specimens: CAS-ICH 239322 and CAS-ICH 239352 (Davidson Seamount, RN Lea and EJ Burton); CAS-ICH 239362 (Sur Ridge, RN Lea and EJ Burton).

Cyclothone signata Garman, 1899. Showy Bristlemouth. Museum specimens: CAS-ICH 239353 (Davidson Seamount, RN Lea and EJ Burton); MLMLF0238 (Monterey Bay, GM Cailliet); SIO 67-102 (within Davidson Seamount Management Zone, CL Hubbs).

Gonostoma atlanticum Norman, 1930. Atlantic Fangjaw. Museum specimen: CASICH 239304 (Davidson Seamount, RN Lea and EJ Burton).

\section{Family STERNOPTYCHIDAE - marine hatchetfishes}

Argyropelecus affinis Garman, 1899. Slender Hatchetfish. Museum specimens: CAS-ICH 239354 (Davidson Seamount, RN Lea and EJ Burton); SIO 67-102 (within Davidson Seamount Management Zone, CL Hubbs).

Argyropelecus hemigymnus Cocco, 1829. Spurred Hatchetfish. Museum specimens: CAS-ICH 239331 and CAS-ICH 239355 (Davidson Seamount, RN Lea and EJ Burton); SIO 67-102 (within Davidson Seamount Management Zone, CL Hubbs).

Argyropelecus lychnus Garman, 1899. Tropical Hatchetfish. Museum specimens: MLMLF0255 (Monterey Bay, ME Anderson); SWFSC uncatalogued (Sur Ridge during May 2015, W Watson).

Argyropelecus sladeni Regan, 1908. Lowcrest Hatchetfish. Museum specimens: CAS-ICH 239356 (Davidson Seamount, RN Lea and EJ Burton); MLMLF0258 (off Santa Cruz, B Antrim); SIO 67-112 (Monterey Canyon, CL Hubbs). Common name follows Hubbs et al. (1979).

Danaphos oculatus (Garman, 1899). Bottlelight. Museum specimens: CAS-ICH 239295 and CAS-ICH 239357 (Davidson Seamount, RN Lea and EJ Burton); SIO 67-102 (within Davidson Seamount Management Zone, CL Hubbs).

Sternoptyx diaphana Hermann, 1781. Longspine Hatchetfish. Museum specimens: CAS-ICH 36778 (Monterey Bay, ME Anderson); CAS-ICH 54992 (Monterey Canyon, ME Anderson); MLMLF0265 (Monterey Bay, GM Cailliet).

Sternoptyx obscura Garman, 1899. Dusky Hatchetfish. Museum specimens: CASICH 239386 (Monterey Canyon, RN Lea and EJ Burton); MLMLF0268 (Monterey Bay, ME Anderson); CAS-SU 63852 (Monterey Canyon, TN Fast). 


\section{Family PHOSICHTHYIDAE - lightfishes}

Vinciguerria lucetia (Garman, 1899). Panama Lightfish. Museum specimen: MLMLF0278 (Monterey Bay, ME Anderson). Specimen re-examined by RN Lea, 2018; $29.5 \mathrm{~mm}$ SL.

\section{Family STOMIIDAE - dragonfishes}

Aristostomias scintillans (Gilbert, 1915). Shiny Loosejaw. Described from Monterey Bay. Museum specimens: Holotype (USNM 75808); CAS-ICH 239365 (Sur Ridge, RN Lea and EJ Burton); CAS-SU 58424 (off Hopkins Marine Station, JC Briggs); CAS-SU 64323 (Monterey Canyon, TN Fast). Publication: Gilbert (1915) (original description).

Bathophilus flemingi Aron and McCrery, 1958. Highfin Dragonfish. Museum specimens: CAS-ICH 36762 (Monterey Bay, ME Anderson), CAS-ICH 56236 (off Point Sur, ME Anderson); CAS-SU 63582 (Monterey Canyon, ME Anderson).

Chauliodus macouni Bean, 1890. Pacific Viperfish. Museum specimens: CAS-ICH 36779 (Monterey Bay, ME Anderson); CAS-ICH 239333 (Davidson Seamount, RN Lea and EJ Burton); CAS-ICH 239366 (Sur Ridge, RN Lea and EJ Burton); SIO 67102 (within Davidson Seamount Management Zone, CL Hubbs).

Idiacanthus antrostomus Gilbert, 1890. Pacific Blackdragon. Museum specimens: CAS-ICH 36746 and CAS-ICH 36782 (Monterey Bay, ME Anderson); CAS-ICH 239316 (Davidson Seamount, RN Lea and EJ Burton); CAS-ICH 239367 (Sur Ridge, RN Lea and EJ Burton); SIO 67-102 (within Davidson Seamount Management Zone, CL Hubbs).

Stomias atriventer Garman, 1899. Blackbelly Dragonfish. Museum specimens: SIO 89-167 (south of Sur Ridge, identifier unknown, NMFS); SIO 78-115 (between Davidson Seamount Management Zone and MBNMS, 6.5 miles east of zone, identifier unknown, CalCOFI) included here due to close proximity of MBNMS and other record.

Tactostoma macropus Bolin, 1939. Longfin Dragonfish. Described from off Monterey Bay. Museum specimens: Holotype (CAS-SU 33325); CAS-ICH 15012 (west of Big Sur, RN Lea); CAS-ICH 36740 (Monterey Bay, ME Anderson); CAS-ICH 239297 (Davidson Seamount, RN Lea and EJ Burton); SIO 67-102 (within Davidson Seamount Management Zone, CL Hubbs). Publication: Bolin (1939) (original description). 


\section{Order Aulopiformes \\ Family SYNODONTIDAE - lizardfishes}

Synodus lucioceps (Ayres, 1855). California Lizardfish. Museum specimens: CASICH 18218 (Santa Cruz, R Dempster); CAS-ICH 79997 (Monterey Beach near Wharf \#2, WC Freihofer); CAS-SU 12604 (Pacific Grove, H Heath).

\section{Family NOTOSUDIDAE - waryfishes}

Scopelosaurus adleri (Fedorov, 1967). Longfin Waryfish. Listed with reservation. Museum specimens: SWFSC uncatalogued (Davidson Seamount during May 2015, W Watson, provisional identification; two specimens: 15.7-30.3 mm SL). Larger specimen identified as $S$. adleri according to pigment characters in Balanov and Savinykh (1999). Smaller specimen identified as Scopelosaurus sp.; pigment characters don't quite fit those given by Balanov and Savinykh (1999) for either S. adleri or $S$. harryi, but are closest to $S$. adleri. This species has a broad distribution in the northern Pacific Ocean, including California (Balanov and Savinykh 1999). This is the first known record of occurrence within Monterey Bay National Marine Sanctuary (unpublished data, Burton, Lea, and DeVogelaere). Common name follows Mecklenburg et al. (2002).

Scopelosaurus harryi (Mead, 1953). Scaly Waryfish. Museum specimens: CAS-ICH 36764 (Monterey Bay, ME Anderson); SIO 74-14 (within Davidson Seamount Management Zone, identifier unknown, CalCOFI).

\section{Family SCOPELARCHIDAE - pearleyes}

Benthalbella dentata (Chapman, 1939). Northern Pearleye. Museum specimens: CAS-ICH 83254 (Monterey Canyon, ME Anderson); CAS-ICH 239368 (Sur Ridge, RN Lea and EJ Burton); SIO 67-112 (Monterey Canyon, CL Hubbs).

\section{Family ALEPISAURIDAE - lancetfishes}

Alepisaurus ferox Lowe, 1833. Longnose Lancetfish. Museum specimens: CAS-ICH 26078 (Half Moon Bay beach during May 1953, identifier unknown); CAS-ICH 26776 (Monterey Bay during May 1960, identifier unknown). Publications: Phillips (1930a) (Fan Shell Beach during Apr 1930, El Niño); Phillips (1931b) (Monterey during Oct 1930, El Niño); Phillips (1932c) (Monterey during 1931, El Niño). Records have coincided with El Niño events, but collections are likely due to beach cast events. According to Phillips (1930a) "This fish is taken only when it is forced above its natural deep sea strata." 


\section{Family ANOTOPTERIDAE - daggerfishes}

Anotopterus nikparini Kukuev, 1998. North Pacific Daggertooth. Museum specimen: SIO 06-16 (Monterey Bay, KA Moots). Previously recognized in family Paralepididae (barracudinas). We follow Voskoboinikova and Nazarkin (2017), and current usage in Catalog of Fishes (Eschmeyer et al. 2017); with placement in family Anotopteridae.

\section{Family PARALEPIDIDAE - barracudinas}

Arctozenus risso (Bonaparte, 1840). White Barracudina. Museum specimens: CASSU 49270 (Monterey Bay, TN Fast); CAS-SU 49271 (Monterey Canyon, RL Bolin).

Lestidiops ringens (Jordan \& Gilbert, 1880). Slender Barracudina. Museum specimens: MLMLF0316 (Monterey Bay, ME Anderson); SIO 67-112 (Monterey Canyon, CL Hubbs); SIO 74-14 (within Davidson Seamount Management Zone, identifier unknown, CalCOFI).

Lestidiops sphyraenopsis Hubbs, 1916. Smalleye Barracudina. Museum specimens: CAS-ICH 18728 (Half Moon Bay, L Dempster); CAS-SU 35171 (10 miles south of Point Sur, identifier unknown, Templeton Crocker Expedition). Common name follows Hubbs et al. (1979).

Magnisudis atlantica (Krøyer, 1868). Duckbill Barracudina. Museum specimen: CAS-ICH 41868 (Monterey Wharf \#2, JB Phillips).

\section{Family BATHYSAURIDAE - deep-sea lizardfishes}

Bathysaurus mollis Günther, 1878. Highfin Lizardfish. Publication: Burton and Lundsten (2008) (Davidson Seamount, based on imagery). Visual records: MBARI/ NOAA T426-08, MBARI/NOAA T427-07 and MBARI/NOAA T427-10 (Davidson Seamount, Jacobson Stout et al. 2017). Previously recognized in family Synodontidae (lizardfishes). We follow Sato and Nakabo (2002), Russell (2003), and current usage in Catalog of Fishes (Eschmeyer et al. 2017), with placement in family Bathysauridae.

\section{Order Myctophiformes} Family MYCTOPHIDAE - lanternfishes

Ceratoscopelus townsendi (Eigenmann \& Eigenmann, 1889). Dogtooth Lampfish. Museum specimens: CAS-ICH 239298 and CAS-ICH 239334 (Davidson Seamount, 
RN Lea and EJ Burton); SIO 67-102 (within Davidson Seamount Management Zone, CL Hubbs).

Diaphus theta Eigenmann and Eigenmann, 1890. California Headlightfish. Museum specimens: CAS-ICH 239307 (Davidson Seamount, RN Lea and EJ Burton); CAS-ICH 239369 (Sur Ridge, RN Lea and EJ Burton); CAS-ICH 239389 (Monterey Canyon, RN Lea and EJ Burton); SIO 67-102 (within Davidson Seamount Management Zone, CL Hubbs).

Diogenichthys atlanticus (Tåning, 1928). Longfin Lanternfish. Museum specimens: CAS-ICH 239336 (Davidson Seamount, RN Lea and EJ Burton); SIO 74-14 (within Davidson Seamount Management Zone, identifier unknown, CalCOFI); CAS-SU 63873 (Monterey Canyon, TN Fast).

Lampanyctus steinbecki Bolin, 1939. Longfin Lampfish. Museum specimens: SIO 74-14 (within Davidson Seamount Management Zone during Aug 1970, identifier unknown, CalCOFI); SIO 89-182 (outside Monterey Bay during Aug 1972, identifier unknown, NMFS); SIO 89-184 (outside Monterey Bay during Aug 1972, identifier unknown, NMFS).

Lampanyctus tenuiformis (Brauer, 1906). lanternfish. Listed with reservation. Museum specimens: SIO 89-165, SIO 89-167, SIO 89-168 and SIO 96-193 (west of Big Sur, identifier unknown, NMFS). Wisner (1976) indicates L. tenuiformis is apparently widespread in the eastern Pacific Ocean, but with few specimens taken is either uncommon or not captured. A large number of SIO specimens are available; however, we consider suspect due to rarity. We tentatively include here, understanding specimens may be misidentified. No official common name.

Nannobrachium regale (Gilbert, 1892). Pinpoint Lampfish. Museum specimens: MLMLF0389 (Monterey Bay, GM Cailliet); SIO 67-102 (within Davidson Seamount Management Zone, CL Hubbs); SWFSC uncatalogued (Sur Ridge during May 2015, W Watson).

Nannobrachium ritteri (Gilbert 1915). Broadfin Lampfish. Described from Monterey Bay. Museum specimens: Holotype (USNM 75807); CAS-ICH 239308 (Davidson Seamount, RN Lea and EJ Burton); CAS-ICH 239370 (Sur Ridge, RN Lea and EJ Burton); SIO 67-102 (within Davidson Seamount Management Zone, CL Hubbs). Publication: Gilbert (1915) (original description).

Parvilux ingens Hubbs and Wisner, 1964. Giant Lampfish. Museum specimens: CAS-ICH 63859 (Monterey Canyon, RL Bolin); MLMLF0397 (Monterey Bay, GM Cailliet); SIO 87-16 (off Point Sur, R Snodgrass). 
Protomyctophum crockeri (Bolin, 1939). California Flashlightfish. Museum specimens: CAS-ICH 239326 (Davidson Seamount, RN Lea and EJ Burton); CAS-ICH 239377 (Monterey Bay, RN Lea and EJ Burton); SIO 67-102 (within Davidson Seamount Management Zone, CL Hubbs).

Protomyctophum thompsoni (Chapman, 1944). Northern Flashlightfish. Museum specimen: CAS-ICH 203156 (Monterey Canyon, RL Bolin).

Stenobrachius leucopsarus (Eigenmann \& Eigenmann, 1890) Northern Lampfish. Museum specimens: CAS-ICH 239359 (Davidson Seamount, RN Lea and EJ Burton); CAS-ICH 239371 (Sur Ridge, RN Lea and EJ Burton); CAS-ICH 239378 (Monterey Bay, RN Lea and EJ Burton); SIO 67-102 (within Davidson Seamount Management Zone, CL Hubbs).

Symbolophorus californiensis (Eigenmann \& Eigenmann, 1889). California Lanternfish. Museum specimens: CAS-ICH 239301 (Davidson Seamount, RN Lea and EJ Burton); MLMLF0412 (Monterey Canyon, ME Anderson); SIO 89-174 (offshore Monterey Bay, identifier unknown, NMFS). Common name follows Hubbs et al. (1979).

Taaningichthys paurolychnus Davy, 1972. Dimlight Lampfish. Museum specimens: Paratypes (SIO 67-102, within Davidson Seamount Management Zone, CL Hubbs).

Tarletonbeania crenularis (Jordan \& Gilbert, 1880). Blue Lanternfish. Museum specimens: CAS-ICH 239392 (Monterey Canyon, RN Lea and EJ Burton); MLMLF0420 (Monterey Bay, D Varoujean); SIO 67-102 (within Davidson Seamount Management Zone, CL Hubbs).

Triphoturus mexicanus (Gilbert, 1890). Mexican Lampfish. Museum specimens: CAS-ICH 239360 (Davidson Seamount, RN Lea and EJ Burton); CAS-ICH 239372 (Sur Ridge, RN Lea and EJ Burton); CAS-SU 66290 (Monterey Canyon, RL Bolin).

\section{Order Lampridiformes}

Family LAMPRIDAE - opahs

Lampris incognitus (Underkoffler, Luers, Hyde, and Craig, 2018). Smalleye Pacific Opah. Museum specimens: CAS-ICH 29716 (off Princeton, Half Moon Bay, identifier unknown, Aug 1973, El Niño); CAS-ICH 53013 (WSW of Point Piedras Blancas, identifier unknown, Oct 1964, moderate El Niño); CAS-ICH 53015 (off Half Moon Bay, identifier unknown, Aug 1973, El Niño). Publication: Radovich (1961) (off Monterey during 1957 and 1959, El Niño). Categorized as occurring during warmwater events (e.g., El Niño). Based on recent genetic, morphological, and meristic data 
analyses, recent publications propose a taxonomic revision of Lampris guttatus (Brünnich, 1788), including a description of the new species Lampris incognitus (Hyde et al. 2014, Underkoffler et al. 2018). We follow Underkoffler et al. (2018), where Lampris incognitus is the only known opah species occurring in the Eastern North Pacific. Previous to this revision, California records have been reported as Lampris regius and Lampris guttatus.

\section{Family TRACHIPTERIDAE - ribbonfishes}

Desmodema lorum Rosenblatt and Butler, 1977. Whiptail Ribbonfish. Museum specimen: SIO 74-14 (within Davidson Seamount Management Zone, identifier unknown, CalCOFI). Visual record: MBARI D668-01 (2 miles north of Davidson Seamount Management Zone, Jacobson Stout et al. 2017) included here due to close proximity of MBNMS and other record.

Trachipterus altivelis Kner, 1859. King-of-the-salmon. Museum specimens: CASICH 49067 (Pacific Grove during Oct 1981, WN Eschmeyer); CAS-ICH 58607 (Davenport during Jun 1986, identifier unknown, NMFS); MLMLF0433 (Monterey Breakwater during Jan 1971, GE Kukowski); CAS-SU 13080 (Monterey beach during Jun 1907, JO Snyder). Publication: Phillips (1932d) (Monterey Bay during 1931, El Nińo).

\section{Order Gadiformes}

\section{Family MACROURIDAE - grenadiers}

Coelorinchus scaphopsis (Gilbert, 1890). Shoulderspot Grenadier. Museum specimen: SIO 88-127 (off Cambria, HG Moser).

Coryphaenoides acrolepis (Bean, 1884). Pacific Grenadier. Museum specimens: CAS-ICH 18780 (off Santa Cruz, R Dempster); CAS-ICH 42566 (off Cypress Point, RN Lea); CAS-ICH 65080 (Monterey Bay, T Iwamoto); CAS-SU 17980 (off Point Lobos, RL Bolin). Publication: Burton and Lundsten (2008) (Davidson Seamount, based on imagery). Visual records: MBARI/NOAA T428-04 and MBARI/NOAA D944-04 (Davidson Seamount, EJ Burton, L Kuhnz, and L Lundsten; Jacobson Stout et al. 2017).

Coryphaenoides armatus (Hector, 1875). Abyssal Grenadier. Publication: Burton and Lundsten (2008) (Davidson Seamount, based on imagery). Visual record: MBARI/NOAA T427-03 (Davidson Seamount, EJ Burton and L Kuhnz). 
Coryphaenoides cinereus (Gilbert, 1896). Popeye Grenadier. Museum specimens: UW 41685 (near Monterey Bay, Hoff 1999); UW 113725 (Monterey Bay, J Hoff). Publication: Hoff (1999) (near Monterey Bay, UW 41685). Common name follows Cohen et al. (1990).

Coryphaenoides filifer (Gilbert, 1896). Threadfin Grenadier. Publication: Burton and Lundsten (2008) (Davidson Seamount, based on imagery). Visual record: MBARI/NOAA T426-04 (Davidson Seamount, EJ Burton and L Kuhnz; Jacobson Stout et al. 2017). Common name follows Hubbs et al. (1979).

Coryphaenoides leptolepis Günther, 1877. Ghostly Grenadier. Publication: Burton and Lundsten (2008) (Davidson Seamount, based on imagery). Visual records: MBARI/NOAA T427-04 and MBARI/NOAA T945-02 (Davidson Seamount, EJ Burton and L Kuhnz; Jacobson Stout et al. 2017).

Coryphaenoides pectoralis (Gilbert, 1892). Giant Grenadier. Museum specimens: CAS-ICH 34356 (Monterey Bay, T Iwamoto); CAS-ICH 51589 (Santa Cruz, RN Lea); MLMLF0459 (Monterey Bay, ME Anderson). Visual records: MBARI/NOAA T943-03 (Davidson Seamount, EJ Burton and T Iwamoto); MBARI D621 (Sur Ridge, EJ Burton and L Kuhnz). Previously recognized as Albatrossia pectoralis. We follow Wilson (1994), Morita (1999), Wilson and Attia (2003), and Gaither et al. (2016), with placement in Coryphaenoides.

Malacocephalus laevis (Lowe, 1843). Softhead Grenadier. Museum specimens: CAS-ICH 55884 (San Simeon, T Iwamoto); UW 41690 (near Point Sur, Hoff 1999). Publication: Hoff (1999) (near Point Sur, UW 41690). Common name follows Cohen et al. (1990).

Nezumia liolepis (Gilbert, 1890). Smooth Grenadier. Museum specimen: CAS-SU 5351 (Cabrillo Canyon, SW of Santa Cruz, identifier unknown, US Fish Commission). Publication: Hoff et al. (2000) (outside Monterey Bay).

Nezumia stelgidolepis (Gilbert, 1890). California Grenadier. Museum specimens: CAS-ICH 30457 (Monterey Bay, ME Anderson); CAS-ICH 31509 (off Point San Simeon, WI Follett); CAS-ICH 32309 (off Pigeon Point, T Iwamoto). Publication: Hoff et al. (2000) (off Cape San Martin).

\section{Family MORIDAE - codlings}

Antimora microlepis Bean, 1890. Pacific Flatnose. Museum specimens: CAS-ICH 34353 and CAS-ICH 34354 (off Monterey Bay, ME Anderson); CAS-SU 5276 (off 
Monterey Bay, identifier unknown, US Fish Commission). Publication: Burton and Lundsten (2008) (Davidson Seamount, based on imagery). Visual record: MBARI/ NOAA T427-05 (Davidson Seamount, L Kuhnz).

Halargyreus johnsonii Günther, 1862. Slender Codling. Museum specimen: UW 48729 (east of Sur Ridge, RC Harrison). Common name follows Cohen et al. (1990).

Physiculus nematopus Gilbert, 1890. Charcoal Codling. Museum specimen: CASICH 241419 (head of Monterey Canyon at Monterey Bay, RN Lea). This account documents the first verifiable record of this species from outside the Panamic biogeographic province. The specimen was collected by the California Department of Fish and Wildlife, F/V Donna Kathleen, on 25 Mar 2013, using fish traps while conducting juvenile rockfish surveys in Monterey Bay. The fish was given to RNL for identification as an unusual capture. Upon examination it was obvious as Physiculus and would have been easy to assume it as Physiculus rastrelliger, Hundred-fathom Codling; the species known from the eastern North Pacific and occasionally encountered off California. However, during routine analysis it became apparent that it was not $P$. rastrelliger and was in fact $P$. nematopus. Data relating to the specimen including characteristics distinguishing it from $P$. rastrelliger: $173 \mathrm{~mm}$ TL; $155 \mathrm{~mm}$ SL; $62.1 \mathrm{~g}$; caudal area injured, hence second dorsal fin, anal fin soft-ray, and caudal fin counts were not possible - confirmed by x-ray; first Dorsal fin IX; Pectoral fin left 24; Pelvic fins 6, filamentous ( 7 in $P$. rastrelliger); Gill rakers left $3+13=16$ (18-22 on lower limb and 26 to 30 total in P. rastrelliger). Paulin (1989) provided a review of the genus Physiculus.

Physiculus rastrelliger Gilbert, 1890. Hundred-fathom Codling. Museum specimens: MLMLF0486 (Monterey Bay, ME Anderson); SIO 09-200 (Monterey Canyon, identifier unknown).

\section{Family MERLUCCIIDAE - merlucciid hakes}

Merluccius productus (Ayres, 1855). Pacific Hake. Museum specimens: CAS-ICH 26079 (Half Moon Bay, WI Follett); CAS-ICH 81648 (Monterey Canyon, EH Ahlstrom); CAS-SU 65856 (Monterey Canyon, TN Fast).

\section{Family GADIDAE - cods}

Gadus chalcogrammus Pallas, 1814. Walleye Pollock. Publications: Phillips (1942a) (Monterey Bay); Phillips (1943b) (off Carmel). Visual records: unpublished data (landed at Moss Landing during 1986, $588 \mathrm{~mm}$ TL, RN Lea); unpublished data (off Santa Cruz during 1996, 165 fathoms, $625 \mathrm{~mm}$ TL, RN Lea); unpublished data (south of Lopez Point during 1997, 220 fathoms, 550 mm TL, RN Lea). 
Gadus macrocephalus Tilesius, 1810. Pacific Cod. Museum specimens: MLMLF0467 and MLMLF0468 (Monterey Bay, E Osada). Publications: Phillips (1951a) (off Point Sur), Phillips (1953) (Monterey area).

Microgadus proximus (Girard, 1854). Pacific Tomcod. Museum specimens: CASICH 25447 (Half Moon Bay, WI Follett); MLMLF0469 (off Pajaro River at Monterey Bay, L Talent); UMMZ 56377 (Monterey Bay, WL Scofield).

\section{Order Ophidiiformes}

Family OPHIDIIDAE - cusk-eels

Chilara taylori (Girard, 1858). Spotted Cusk-eel. Described from Monterey. Museum specimens: Syntypes [MCZ 35928, UMMZ 146978, USNM 867]; CAS-ICH 31773 (off Cambria, JB Phillips); CAS-ICH 81604 (Monterey, RL Bolin); CAS-ICH 236552 (Del Monte Beach, RN Lea); CAS-SU 64112 (Davidson Seamount, 82 mm TL nektonic prejuvenile, specimen likely spit up by Albacore, collected by fisherman, RN Lea). Publications: Girard (1858a) (original description); Cailliet et al. (1977) (Hwy 1 Bridge at Elkhorn Slough).

Lamprogrammus niger Alcock, 1891. Paperbone Cusk-eel. Museum specimen: UW 47396 (canyon south of Pioneer Canyon, J Hoff).

Luciobrotula sp. A. cusk-eel. Publications: Burton and Lundsten (2008) (Davidson Seamount, based on imagery); Burton et al. (2017) (Sur Ridge, based on imagery). Visual records: MBARI T0357-02 and MBARI T0611-02 (Monterey Canyon, Jacobson Stout et al. 2017); MBARI D0981-02 (Sur Ridge, Burton et al. 2017); MBARI/NOAA T0430-11 (Davidson Seamount, Burton and Lundsten 2008). Taxonomist assessment of MBARI visual records in Aug 2012 by Jørgen G. Nielsen (retired, University of Copenhagen); 'closest recorded species in this genus is from Panama, so these are probably an undescribed species' (Jacobson Stout et al. 2017). No official common name.

Spectrunculus grandis (Günther, 1877). Giant Cusk-eel. Publications: Burton and Lundsten (2008), and Lundsten et al. (2009) (Davidson Seamount, based on imagery). Visual records: MBARI/NOAA T946-01, MBARI/NOAA T946-03, MBARI/NOAA T946-04 and MBARI/NOAA T947-01 (Davidson Seamount, EJ Burton, GM Cailliet and L Lundsten).

\section{Family BYTHITIDAE - viviparous brotulas}

Brosmophycis marginata (Ayres, 1854). Red Brotula. Museum specimens: CASICH 80641 (off Half Moon Bay, B Breen); SIO 89-194 (west of Monterey Bay, identifier unknown, NMFS). 
Cataetyx rubrirostris Gilbert, 1890. Rubynose Brotula. Museum specimens: CASICH 36749, CAS-ICH 36771, and CAS-ICH 36792 (Monterey Bay, ME Anderson). Publications: Anderson et al. (1979) (Monterey Bay); Gibbs (1991) (Monterey and Carmel Canyons).

\section{Order Batrachoidiformes}

Family BATRACHOIDIDAE - toadfishes

Porichthys notatus Girard, 1854. Plainfin Midshipman. Museum specimens: CASICH 23406 (Santa Cruz Wharf, RN Lea); CAS-ICH 233552 (Monterey Bay, RR Rofen); CAS-SU 58446 (Elkhorn Slough, JC Briggs). Publication: Yoklavich et al. (1991) (Hwy 1 Bridge, Dairy, Kirby Park, and Hudson's Landing at Elkhorn Slough).

\section{Order Lophiiformes}

Family LOPHIIDAE - goosefishes

Lophiodes spilurus (Garman, 1899). Threadfin Goosefish. Museum specimen: LACM 43535.001 (SW of Santa Cruz during 1983, El Niño, RN Lea). Publication: Lea et al. (1984) (SW of Santa Cruz during 1983, El Nińo, second California record). Categorized as occurring during warm-water events (e.g., El Niño).

\section{Family CHAUNACIDAE - gapers}

Chaunacops coloratus (Garman, 1899). gaper. Museum specimen: CAS-ICH 216055 (Davidson Seamount, GM Cailliet and HC Ho). Publications: Burton and Lundsten (2008) (Davidson Seamount, CAS-ICH 216055); Lundsten et al. (2012) (Davidson Seamount, CAS-ICH 216055). No official common name.

\section{Family MELANOCETIDAE - blackdevils}

Melanocetus johnsonii Günther, 1864. Blackdevil. Museum specimen: UW 48693 (Sur Canyon, identifier unknown, NMFS). Visual record: MBARI D695 (Monterey Bay, Jacobson Stout et al. 2017). MBARI visual record resolved to genus; however, M. johnsonii is the only known species off California (Fitch and Lavenberg 1968, Love et al. 2005).

\section{Family HIMANTOLOPHIDAE - footballfishes}

Himantolophus nigricornis Bertelsen and Krefft, 1988. footballfish. Museum specimen: SIO 94-71 (off Point Año Nuevo, RN Lea). No official common name. 
Himantolophus sagamius (Tanaka, 1918). Pacific Footballfish. Museum specimen: CAS-ICH 57639 (Monterey Canyon, E Bertelsen). Publication: Lea (1988) (Monterey Canyon, CAS-ICH 57639).

\section{Family ONEIRODIDAE - dreamers}

Chaenophryne longiceps Regan, 1925. dreamer. Museum specimens: SIO 98-115 (Monterey Bay, RN Lea); USNM 150087 (off Point Pinos, identifier unknown, "Albatross" Explorations of the California Coast, 1904). No official common name.

Chaenophryne melanorhabdus Regan and Trewavas, 1932. Smooth Dreamer. Museum specimens: SIO 96-75 off (Moss Landing, B Leos); SIO 97-128 (south of Point Sur, identifier unknown, CDFG). Common name follows Mecklenburg et al. (2002).

\section{Family CERATIIDAE - seadevils}

Cryptopsaras conesii Gill, 1883. Triplewart Seadevil. Museum specimen: CASICH 73320 (Monterey Canyon during Aug 1990, M Innes). Publication: Fast (1957) (Monterey Bay during Sept 1956, warm-water event). Categorized as occurring during warm-water events (e.g., El Niño).

\section{Order Mugiliformes}

\section{Family MUGILIDAE - mullets}

Mugil cephalus Linnaeus, 1758. Striped Mullet. Museum specimens: SBMNH 1800 (Marina Dunes, identifier unknown); USNM 26796 (Monterey, DS Jordan). Visual records: unpublished data (Monterey Wharf \#2 during Oct 1987, K Oda); unpublished data (Monterey Wharf \#2 during Oct 2001, 545 mm TL, 1880.2 g, RN Lea). Found in warm seas (Miller and Lea 1972), rare north of Point Conception (Moyle 2002); and found in San Francisco Bay and as far north as Humboldt Bay (Wallace et al. 2015) during warm water years (e.g. El Niño). Categorized as occurring during warm-water events (e.g., El Niño).

\section{Order Atheriniformes} Family ATHERINOPSIDAE - New World silversides

Atherinops affinis (Ayres, 1860). Topsmelt. Museum specimens: CAS-ICH 45119 (southern Monterey Bay, D Wilson); CAS-SU 55244 (Elkhorn Slough, WC Freihofer); CAS-SU 58401 (Elkhorn Slough, JC Briggs). Publication: Yoklavich et al. (1991) (Hwy 1 Bridge, Dairy, Kirby Park, and Hudson's Landing at Elkhorn Slough). 
Atherinopsis californiensis Girard, 1854. Jacksmelt. Museum specimens: MLMLF0546 (near Dairy at Elkhorn Slough, GE Kukowski); CAS-SU 47508 (Pacific Grove, WC Freihofer); CAS-SU 58399 (Elkhorn Slough, JC Briggs). Publication: Yoklavich et al. (1991) (Hwy 1 Bridge, Dairy, and Kirby Park at Elkhorn Slough).

Leuresthes tenuis (Ayres, 1860). California Grunion. Museum specimens: CAS-ICH 42072 (New Brighton State Beach, Santa Cruz County, JD Spratt); CAS-ICH 54825 (Moss Landing during 1983, El Niño, GM Cailliet); SIO 45-56 (off Del Monte Beach, CL Hubbs). Publications: Thompson (1920) (Monterey); Phillips (1943a) (Monterey during 1942); Radovich (1961) (Monterey during 1959, El Niño); Spratt (1981) (New Brighton State Beach, Santa Cruz County, CAS-ICH 42072). Categorized as occurring during warm-water events (e.g., El Niño).

\section{Order Beloniformes \\ Family EXOCOETIDAE - flyingfishes}

Cheilopogon pinnatibarbatus (Bennett, 1831). Smallhead Flyingfish. Publication: Cooper (1863) (to at least Santa Cruz). Visual records: Observed during Aug 1986 off Point Joe by John Stern (San Francisco State University graduate student, reported to Alan Baldridge, Hopkins Marine Station); three sightings observed from Cypress Point and Point Sur areas during Jun 1992 (warm-water event) by Richard Ternullo (skipper of PT SUR CLIPPER, reported to Alan Baldridge, Hopkins Marine Station); observed 2.5 miles off Cypress Point during Jul 1994 (warm-water event) by Nancy Black (Oceanic Society Expeditions, reported to RN Lea). Categorized as occurring during warm-water events (e.g., El Niño).

\section{Family BELONIDAE - needlefishes}

Strongylura exilis (Girard, 1854). California Needlefish. Museum specimen: CASICH 64942 (San Francisco Bay, near Golden Gate Bridge during Apr 1986, moderate El Niño, identifier unknown). Publication: Phillips (1940) ("short distance off Monterey”). Categorized as occurring during warm-water events (e.g., El Niño).

\section{Family SCOMBERESOCIDAE - sauries}

Cololabis saira (Brevoort, 1856). Pacific Saury. Museum specimens: CAS-ICH 18215 (Half Moon Bay, RR Harry); CAS-ICH 25629 (off Waddell Creek during 1950, WI Follett); MLMLF0555 (Monterey Bay during Nov 1970, L Talent). Visual 
record: SIMoN (2018) (Davidson Seamount during Jul 2018, EJ Burton). Publication: Phillips (1932a) (Monterey Bay during 1931, El Nińo).

\section{Order Stephanoberyciformes Family MELAMPHAIDAE - bigscales}

Melamphaes lugubris Gilbert, 1890. Highsnout Bigscale. Museum specimens: CASICH 36796 (Monterey Bay, ME Anderson); SIO 67-102 (within Davidson Seamount Management Zone, CL Hubbs); SWFSC uncatalogued (Davidson Seamount during May 2015, W Watson). We collected a larval specimen of Melamphaes sp. from Davidson Seamount during 2015, that was provisionally identified by W Watston (NOAA Fisheries, SWFSC) as Melamphaes cf. parvus. Identification follows Sandknop and Watson (1996). Specimen may be Melamphaes parvus; however, not in good enough condition to be certain. This species is known to occur off California (Ebeling 1962, Sandknop and Watson 1996, Kotlyar 2004). This provisional identification of M. parvus is not included in the total number of species in MBNMS. Common name follows Hubbs et al. (1979).

Poromitra crassiceps (Günther, 1878). Crested Bigscale. Museum specimens: CASICH 36752 (Monterey Bay, ME Anderson); CAS-ICH 239361 (Davidson Seamount, RN Lea and EJ Burton); SIO 67-102 (within Davidson Seamount Management Zone, CL Hubbs). Common name follows Hubbs et al. (1979).

Scopelogadus bispinosus (Gilbert, 1915). Twospine Bigscale. Museum specimens: CAS-ICH 36735 and CAS-ICH 36790 (Monterey Bay, ME Anderson); SIO 67-102 (within Davidson Seamount Management Zone, CL Hubbs). Common name follows Hubbs et al. (1979).

\section{Order Beryciformes}

Family ANOPLOGASTRIDAE - fangtooths

Anoplogaster cornuta (Valenciennes, 1833). Fangtooth. Museum specimens: CASICH 55413 (off Point Sur, ME Anderson); SIO 88-99 (south of Point Sur, identifier unknown, NMFS).

\section{Order Zeiformes}

\section{Family OREOSOMATIDAE - oreos}

Allocyttus folletti Myers, 1960. Oxeye Oreo. Museum specimens and Publication: CAS-ICH 39087 (off Cypress Point, Anderson et al. 1979); CAS-ICH 39088 (off Point Sur, Anderson et al. 1979). 


\section{Family ZEIDAE - dories}

Zenopsis nebulosa (Temminck \& Schlegel, 1845). Mirror Dory. Museum specimen: CAS-ICH 24164 (Monterey Bay, P Gregory).

\section{Order Gasterosteiformes Family AULORHYNCHIDAE - tubesnouts}

Aulorhynchus flavidus Gill, 1861. Tubesnout. Museum specimens: CAS-SU 35360 (Point Cabrillo at Monterey Bay, RL Bolin); CAS-SU 35361 (Monterey Bay, RL Bolin); CAS-SU 58405 (Carmel Beach, JC Briggs).

\section{Family GASTEROSTEIDAE - sticklebacks}

Gasterosteus aculeatus Linnaeus, 1758. Threespine Stickleback. Museum specimens: OSUM 71680 (Elkhorn Slough, G Varney and G Varney, Jr); CAS-SU 15026 (upper Elkhorn Slough, HW Freeman and IL Firschein). Publication: Yoklavich et al. (1991) (station location from Barry 1983, Hudson's Landing at Elkhorn Slough)

\section{Family SYNGNATHIDAE - pipefishes}

Cosmocampus arctus (Jenkins \& Evermann, 1889). Snubnose Pipefish. Museum specimens: SIO 11-335 (Elkhorn Slough, RN Lea); SIO $45-53$ (Elkhorn Slough, CL Hubbs and RL Bolin).

Syngnathus californiensis Storer, 1845. Kelp Pipefish. Museum specimens: CAS-SU 35362 (Monterey Bay, RL Bolin); CAS-SU 36462 (Elkhorn Slough, ES Herald and WI Follett); CAS-SU 58461 (Hopkins Marine Station, JC Briggs).

Syngnathus exilis (Osburn \& Nichols, 1916). Barcheek Pipefish. Museum specimens: SIO 45-56 (Monterey Bay, Hubbs), SIO 92-135 (Elkhorn Slough, RN Lea and L Allen).

Syngnathus leptorhynchus Girard, 1854. Bay Pipefish. Museum specimens: SIO 45-53 and SIO 62-510 (Elkhorn Slough, CL Hubbs); SIO 93-189 (Elkhorn Slough, RN Lea). Publication: Yoklavich et al. (1991) (Hwy 1 Bridge and Dairy at Elkhorn Slough). 


\section{Order Scorpaeniformes \\ Family SCORPAENIDAE - scorpionfishes}

Scorpaena guttata Girard, 1854. California Scorpionfish. Described from Monterey Bay. Museum specimens: Holotype (USNM 350); MLMLF0613 (off Santa Cruz at Monterey Bay during 1970, L Talent and D Varoujean). Publications: Girard (1854) (original description); Varoujean (1972) (3 miles off Point Santa Cruz, MLMLF0613). Visual record: Observed and collected live at 2 miles from Moss Landing jetty on south side of Monterey Canyon during Dec 1984 by Bill Frogue (commercial fisherman, reported and delivered to Dave Powell for display at Monterey Bay Aquarium).

Sebastes atrovirens (Jordan \& Gilbert, 1880). Kelp Rockfish. Museum specimens: CASICH 25901 (Monterey Bay, WI Follett); CAS-ICH 47396 (kelp bed off Lovers Point, RN Lea); CAS-ICH 56343 (Monterey Breakwater, W Laroche). Publications: Cailliet et al. (1977) (Dairy at Elkhorn Slough); Lea (1983) (Monterey Bay to San Simeon).

Sebastes auriculatus Girard, 1854. Brown Rockfish. Museum specimens: CAS-ICH 14803 (Monterey Bay, DW Behrens); CAS-ICH 40643 (Elkhorn Slough, RL Bolin); CAS-ICH 56272 (Half Moon Bay, W Laroche). Publication: Yoklavich et al. (1991) (Hwy 1 Bridge, Dairy, and Kirby Park at Elkhorn Slough).

Sebastes aurora (Gilbert, 1890). Aurora Rockfish. Museum specimens: CAS-ICH 37500 (west of Pigeon Point, T Iwamoto); CAS-ICH 39893 (off Yankee Point, WN Eschmeyer and T Iwamoto); CAS-ICH 40033 (NW of Point Pinos, WC Ruark).

Sebastes babcocki (Thompson, 1915). Redbanded Rockfish. Museum specimens: CASICH 30468 (Monterey Bay, ME Anderson); CAS-ICH 30735 (west of Moss Landing Marine Laboratories, ME Anderson); CAS-ICH 38724 (Monterey Bay, WA Laroche).

Sebastes borealis Barsukov, 1970. Shortraker Rockfish. Museum specimen: SIO 01186 (between Carmel Bay and Big Sur, RN Lea).

Sebastes brevispinis (Bean, 1884). Silvergray Rockfish. Museum specimens: CASICH 25997 (off Point Sur, identifier unknown); CAS-SU 47300 (off Point Sur, L Schultz).

Sebastes carnatus (Jordan \& Gilbert, 1880). Gopher Rockfish. Described from Monterey Bay. Museum specimens: Syntypes [ANSP 12190 (1), USNM 26993 (5)]; CASICH 14735 (Carmel River Beach, L Hallacher); CAS-ICH 27697 (off Half Moon Bay, WN Eschmeyer). Publication: Jordan and Gilbert (1880c) (original description). 
Sebastes caurinus Richardson, 1844. Copper Rockfish. Museum specimens: CASICH 14734 (Monastery Beach, L Hallacher); CAS-ICH 27356 (Del Monte kelp bed at Monterey Bay, DC Powell); CAS-ICH 40641 (Elkhorn Slough, WA Laroche). Publication: Cailliet et al. (1977) (Hwy 1 Bridge and Dairy at Elkhorn Slough).

Sebastes chlorostictus (Jordan \& Gilbert, 1880). Greenspotted Rockfish. Described from deep water at Monterey; obtained at San Francisco market. Museum specimens: Syntypes (numerous specimens, including: ANSP 12126, USNM 26964, USNM 27092); CAS-ICH 25991 (off Half Moon Bay, WI Follett); CAS-ICH 47400 (off Point Piedras Blancas, RN Lea). Publication: Jordan and Gilbert (1880i) (original description).

Sebastes chrysomelas (Jordan \& Gilbert, 1881). Black-and-yellow Rockfish. Described from Monterey Bay. Museum specimens: Syntypes [numerous specimens including: ANSP 12127 (1), USNM 26968 (4)]; CAS-SU 15122 (Soberanes Point, C Hubbs); CAS-ICH 51863 (just south of Point Sur, RN Lea). Publication: Jordan and Gilbert (1881) (original description).

Sebastes constellatus (Jordan \& Gilbert, 1880). Starry Rockfish. Museum specimens: CAS-ICH 84359 (near Monterey, D Montgomery); CAS-SU 3236 (Pacific Grove, WW Thoburn); CAS-SU 11488 (Monterey, WW Thoburn).

Sebastes crameri (Jordan, 1897). Darkblotched Rockfish. Museum specimens: CAS-ICH 37537 (west of Point Sur, WN Eschmeyer); CAS-ICH 47405 (Monterey Bay, WA Laroche and S Moreland); MLMLF0661 (Monterey Bay, ME Anderson).

Sebastes dallii (Eigenmann \& Beeson, 1894). Calico Rockfish. Described from Monterey; obtained at San Francisco market. Museum specimens: Lectotype [CAS-SU 3893 (drawn specimen), Lectotype established (as figured specimen) in Jordan 1896]; CAS-ICH 217649 (SSE of Point Santa Cruz at Monterey Bay, RN Lea). Publications: Eigenmann and Beeson (1894) (original description); Jordan (1896) (lectotype established); Cailliet et al. (1977) (Dairy at Elkhorn Slough).

Sebastes diaconus Frable, Wagman, Frierson, Aguilar, and Sidlauskas, 2015. Deacon Rockfish. Museum specimens: CAS-SU 11776 (Monterey, DS Jordan); CAS-SU 15112 (Pacific Grove, C Hubbs); UW 40703 (Monterey Bay, WI Follett). Publication: Frable et al. (2015) (new species description, non-type material examined from MBNMS, including Monterey and Pacific Grove listed above). Common name follows Frable et al. (2015).

Sebastes diploproa (Gilbert, 1890). Splitnose Rockfish. Museum specimens: CASICH 37538 (west of Point Sur, K Hakanson); CAS-ICH 39846 (off Point Pinos, PA Gregory); CAS-SU 39916 (Monterey Bay, WA Laroche). 
Sebastes elongatus Ayres, 1859. Greenstriped Rockfish. Museum specimens: CASICH 51203 (Ascension Canyon shelf and slope, S Moreland); CAS-SU 11490 (Monterey, WW Thoburn); CAS-SU 11774 (Monterey, DS Jordan).

Sebastes emphaeus (Starks, 1911). Puget Sound Rockfish. Museum specimens: Monterey Bay Aquarium live specimens (Point Pinos, J Welsh and RN Lea). Two live specimens collected west of Point Pinos (Nov 2014, 225 feet; and Nov 2015, 220 feet) and kept alive on display at Monterey Bay Aquarium (pers. comm. Joe Welsh, Monterey Bay Aquarium).

Sebastes ensifer Chen, 1971. Swordspine Rockfish. Museum specimens: Paratype (SIO 55-97, Monterey); CAS-ICH 42548 (Monterey Canyon, RN Lea); MLMLF0671 (Carmel Bay, LL Smith).

Sebastes entomelas (Jordan \& Gilbert, 1880). Widow Rockfish. Described from deep water outside Monterey Bay. Museum specimens: Syntypes [USNM 27044 (orig. 3, now 2)]; CAS-ICH 14865 (off Half Moon Bay, L Hallacher); CAS-ICH 56949 (off Lovers Point, RN Lea); CAS-SU 15102 (Pacific Grove, C Hubbs). Publication: Jordan and Gilbert (1880f) (original description).

Sebastes eos (Eigenmann \& Eigenmann, 1890). Pink Rockfish. Museum specimens: CAS-ICH 39847 (off Point Pinos, PA Gregory); MLMLF0673 (Monterey Bay, ME Anderson); SIO 07-183 (Ascension Canyon, RN Lea).

Sebastes flavidus (Ayres, 1862). Yellowtail Rockfish. Museum specimens: CAS-ICH 26761 (north of Bird Rock at Asilomar, WI Follett); CAS-SU 4125 (Monterey, DS Jordan); CAS-SU 11471 (Monterey, CH Gilbert).

Sebastes gilli (Eigenmann, 1891). Bronzespotted Rockfish. Publication: Phillips (1957) (Monterey).

Sebastes goodei (Eigenmann \& Eigenmann, 1890). Chilipepper. Museum specimens: CAS-ICH 27358 (off Santa Cruz, RL Bolin); CAS-ICH 37521 (west of Point Sur, WN Eschmeyer); CAS-ICH 40331 (SSW of Point Piedras Blancas, WC Ruark).

Sebastes helvomaculatus Ayres, 1859. Rosethorn Rockfish. Museum specimens: CAS-ICH 42549 (west edge of Monterey Canyon, RN Lea); SIO 00-83 (Bixby Bridge, Monterey County, RN Lea); SIO 98-116 (Carmel Bay, RN Lea).

Sebastes hopkinsi (Cramer, 1895). Squarespot Rockfish. Described from Pacific Grove. Museum specimens: Lectotype (CAS-SU 3232, Lectotype established in Jordan 1896); Paralectotypes [CAS-SU 2282 (3, Pacific Grove); CAS-SU 3112 (Monte- 
rey Bay]; CAS-SU 4146 (Monterey, CH Gilbert). Publications: Cramer (1895) (original description); Jordan (1896) (lectotype established).

Sebastes jordani (Gilbert, 1896). Shortbelly Rockfish. Museum specimens: CASICH 25879 (Monterey Bay, WI Follett); CAS-ICH 37522 (west of Point Sur, KR Hakanson); CAS-ICH 37504 (west of Pigeon Point, T Iwamoto).

Sebastes levis (Eigenmann \& Eigenmann, 1889). Cowcod. Museum specimens: CASICH 37503 (west of Pigeon Point, WN Eschmeyer); CAS-ICH 79580 (Carmel Bay, D Ventresca, Hausehildt, and J Guererro); CAS-SU 11492 (Monterey, CH Gilbert); SWFSC uncatalogued (Davidson Seamount during May 2015, W Watson).

Sebastes macdonaldi (Eigenmann \& Beeson, 1893). Mexican Rockfish. Museum specimen: CAS-ICH 217644 (Santa Cruz, Sept 2002, identifier unknown, CDFG). Publications: Phillips (1961) (off Point Sur during 1959, El Niño); Radovich (1961) (Point Sur during 1959, El Niño). Categorized as occurring during warm-water events (e.g., El Niño).

Sebastes maliger (Jordan \& Gilbert, 1880). Quillback Rockfish. Museum specimens: CAS-ICH 39857 (north of Malpaso Creek, RN Lea); CAS-ICH 51181 (off Davenport, S Moreland); CAS-SU 11497 (Monterey, WW Thoburn).

Sebastes melanops Girard, 1856. Black Rockfish. Museum specimens: CAS-ICH 25885 (Pacific Grove, WI Follett); CAS-ICH 42368 (Soberanes Point tidepool, WA Laroche); CAS-SU 15133 (Del Monte Beach, C Hubbs). Publication: Cailliet et al. (1977) (Dairy at Elkhorn Slough).

Sebastes melanostictus (Matsubara, 1934). Blackspotted Rockfish. Publication: Orr and Hawkins (2008). Authors provide evidence to resurrect the species; distinguishing it from the more northern S. aleutianus (Rougheye Rockfish). Sebastes melanostictus (Blackspotted Rockfish) ranges from central Japan, through Aleutian Islands and Bering Sea, to southern California, and collected at depths from 84 to at least $490 \mathrm{~m}$. No museum specimens known from MBNMS. Because of previous confusion with species of the Rougheye Rockfish complex, museum specimens may be incorrectly identified. Because of the recent species description and geographic range of examined specimens (to the north and south of MBNMS, Orr and Hawkins 2008), we include the species on the list.

Sebastes melanostomus (Eigenmann \& Eigenmann, 1890). Blackgill Rockfish. Museum specimens: CAS-ICH 37534 (off Yankee Point, WN Eschmeyer); CAS-ICH 37539 (west of Sur Ridge, WN Eschmeyer); SIO 00-46 (Carmel Bay, RN Lea).

Sebastes miniatus (Jordan \& Gilbert, 1880). Vermilion Rockfish. Described from Monterey and Santa Barbara. Museum specimens: Syntypes [several specimens includ- 
ing: USNM 26965 (orig. 17, now 3), ZMB 11710 (1)]; CAS-ICH 79586 (off Piedras Blancas, RN Lea); CAS-SU 5552 (Pacific Grove, EC Starks). Publication: Jordan and Gilbert (1880b) (original description).

Sebastes mystinus (Jordan \& Gilbert, 1881). Blue Rockfish. Museum specimens: Paralectotype [off Monterey, USNM 26971]; CAS-ICH 14806 (Monterey Bay, DW Behrens); CAS-ICH 25884 (Pacific Grove, WI Follett); LACM 50157.002 (north of San Simeon, BW Walker and class). Publications: Jordan and Gilbert (1881) (original description); Yoklavich et al. (1991) (Hwy 1 Bridge and Dairy at Elkhorn Slough); Frable et al. (2015) (re-described species, and established lectotype from San Francisco).

Sebastes nebulosus Ayres, 1854. China Rockfish. Museum specimens: SIO 65-355 (1 mile off Point Joe, HG Moser); CAS-SU 4149 (Monterey, DS Jordan); CAS-SU 11549 (Monterey, WW Thoburn).

Sebastes nigrocinctus Ayres, 1859. Tiger Rockfish. Museum specimen: SIO 91-113 (Monterey Bay, N Lai and J Butler).

Sebastes ovalis (Ayres, 1862). Speckled Rockfish. Museum specimens: CAS-ICH 47398 (off Point Piedras Blancas, RN Lea); CAS-ICH 54857 (off Carmel Bay, RN Lea); CAS-SU 4429 (Monterey, DS Jordan).

Sebastes paucispinis Ayres, 1854. Bocaccio. Museum specimens: CAS-ICH 25878 (Monterey Bay, WI Follett); CAS-ICH 40639 (SW of Soberanes Point, WA Laroche); CAS-ICH 234248 (Elkhorn Slough, DW Behrens); SWFSC uncatalogued (Davidson Seamount during May 2015, W Watson). Publication: Yoklavich et al. (1991) (Hwy 1 Bridge and Dairy at Elkhorn Slough).

Sebastes phillipsi (Fitch, 1964). Chameleon Rockfish. Publications: Phillips (1957) ("Hybrid A," described in Fitch 1964, Monterey during 1939); Fitch (1964) (Monterey during 1939).

Sebastes pinniger (Gill, 1864). Canary Rockfish. Museum specimens: CAS-SU 10007 (Pacific Grove, WW Thoburn); CAS-SU 10015 (Monterey, WW Thoburn); CAS-SU 11469 (Monterey, CH Gilbert).

Sebastes proriger (Jordan \& Gilbert, 1880). Redstripe Rockfish. Described from Monterey Bay and Farallones. Museum specimens: Syntypes [USNM 26980 (3), USNM 27105 (4)]; CAS-ICH 66759 (off Yankee Point, RN Lea); CAS-ICH 217645 (off Point Sur, identifier unknown). Publication: Jordan and Gilbert (1880l) (original description).

Sebastes rastrelliger (Jordan \& Gilbert, 1880). Grass Rockfish. Museum specimens: CAS-ICH 40642 (Elkhorn Slough, WA Laroche); CAS-SU 11494 (Monterey, WW 
Thoburn); CAS-SU 15138 (Elkhorn Slough, C Hubbs). Publication: Cailliet et al. (1977) (Hwy 1 Bridge, Dairy, and Kirby Park at Elkhorn Slough).

Sebastes rosaceus Girard, 1854. Rosy Rockfish. Museum specimens: CAS-ICH 33507 (kelp bed off Carmel River, L Hallcher); CAS-ICH 66327 (north of Bird Rock at Asilomar, WI Follett); CAS-SU 4154 (Monterey, DS Jordan).

Sebastes rosenblatti Chen, 1971. Greenblotched Rockfish. Museum specimens: CAS-ICH 39848 (off Point Pinos, PA Gregory); MLMLF0772 (Monterey Bay, ME Anderson); SIO 09-194 (Monterey Canyon, RN Lea).

Sebastes ruberrimus (Cramer, 1895). Yelloweye Rockfish. Museum specimens: SIO 01-111 (Partington Canyon, RN Lea); SIO 09-194 (Montery Canyon, P Reilly); CAS-SU 4380 (Monterey, DS Jordan).

Sebastes rubrivinctus (Jordan \& Gilbert, 1880). Flag Rockfish. Described from Monterey and Santa Barbara. Museum specimens: Syntypes [USNM 26989 (5)]; MLMLF0776 (off Davenport, D Varoujean); MLMLF0777 (off Moss Landing, GM Cailliet). Publication: Jordan and Gilbert (1880i) (original description).

Sebastes rufus (Eigenmann \& Eigenmann, 1890). Bank Rockfish. Museum specimens: CAS-ICH 47379 (west of Point Sur, RN Lea); CAS-ICH 47394 (WNW of Pigeon Point, RN Lea); CAS-ICH 51854 (off Davenport, RN Lea).

Sebastes saxicola (Gilbert, 1890). Stripetail Rockfish. Museum specimens: CASICH 37481 (off Santa Cruz, T Iwamoto); CAS-ICH 51855 (off Monterey Coast Guard Pier, RN Lea); CAS-ICH 56273 (off Soberanes Point, W Laroche).

Sebastes semicinctus (Gilbert, 1897). Halfbanded Rockfish. Museum specimens: CAS-ICH 42551 (Monterey Bay, RN Lea); SIO 85-154 (off Half Moon Bay, D Gibson); SIO 85-155 (Pigeon Point, D Gibson).

Sebastes serranoides (Eigenmann \& Eigenmann, 1890). Olive Rockfish. Museum specimens: CAS-ICH 40657 (Elkhorn Slough, WA Laroche); CAS-ICH 42366 (Half Moon Bay, WA Laroche); CAS-ICH 56954 (Carmel Bay, RN Lea); CAS-SU 15135 (Del Monte Beach, C Hubbs).

Sebastes serriceps (Jordan \& Gilbert, 1880). Treefish. Museum specimens: CAS-ICH 79585 (off Point Joe during 1980, identifier unknown); CAS-SU 11767 (Monterey during Dec 1895, DS Jordan); USNM 110781 (Monterey, DS Jordan).

Sebastes simulator Chen, 1971. Pinkrose Rockfish. Museum specimens: SIO 00-58 (Carmel Bay, RN Lea); SIO 85-73 (Point Sur, W Wakefield); SIO 96-71 (Monterey Bay, S Melton). 
Sebastes umbrosus (Jordan \& Gilbert, 1882). Honeycomb Rockfish. Museum specimen: CAS-ICH 14990 (Point Pinos during Sept 1971, RN Lea).

Sebastes wilsoni (Gilbert, 1915). Pygmy Rockfish. Described from Monterey Bay. Museum specimens: Holotype (USNM 75811); Paratype (CAS-SU 22992); CASICH 15052 (SW of Santa Cruz, JE Fitch); CAS-ICH 47386 (off Point Sur, RN Lea). Publication: Gilbert (1915) (original description).

Sebastes zacentrus (Gilbert, 1890). Sharpchin Rockfish. Museum specimens: CASICH 50020 (NW of Point Piedras Blancas, RN Lea); SIO 09-197 (Soquel Canyon, identifier unknown); SIO 96-71 (Monterey Bay, S Melton).

Sebastolobus alascanus Bean, 1890. Shortspine Thornyhead. Museum specimens: CAS-ICH 37536 (west of Yankee Point, WN Eschmeyer); CAS-ICH 236649 (off Monterey Bay, RL Bolin); CAS-SU 65811 (Monterey Canyon, TN Fast). Publication: Burton and Lundsten (2008) (Davidson Seamount, based on imagery). Visual record: MBARI/NOAA T428-03 (Davidson Seamount, EJ Burton, GM Cailliet, T Trejo).

Sebastolobus altivelis Gilbert, 1896. Longspine Thornyhead. Museum specimens: SIO 67-102 (within Davidson Seamount Management Zone, CL Hubbs); CAS-SU 5270 (SW of Santa Cruz, identifier unknown, US Fish Commission); CAS-SU 63869 (off Carmel, RL Bolin); CAS-SU 65816 (Monterey Canyon, TN Fast).

\section{Family TRIGLIDAE - searobins}

Prionotus stephanophrys Lockington, 1881. Lumptail Searobin. Museum specimens: KU 23710 (Monterey Bay during 1993, El Niño, M Domeier); MLMLF0818 (Monterey Bay during Aug 1999, B Banks). Publication: Lea and Rosenblatt (2000) (Monterey Bay during Oct 1998 and Aug 1999, El Nińo). Categorized as occurring during warm-water events (e.g., El Niño).

\section{Family ANOPLOPOMATIDAE - sablefishes}

Anoplopoma fimbria (Pallas, 1814). Sablefish. Museum specimens: SIO 05-89 (off Lucia, D Kamikawa); SIO 67-108 (SW of Santa Cruz, CL Hubbs); SIO 85-154 (off Half Moon Bay, D Gibson); SIO 91-113 (Monterey Bay, N Lai and J Butler). Publication: Cox (1948) (Monterey).

Erilepis zonifer (Lockington, 1880). Skilfish. Described from Monterey. Museum specimens: Holotype (USNM 27111); CAS-ICH 27079 (SW of Point Santa Cruz, JB Phillips); CAS-ICH 39596 (SSW of Point Santa Cruz, DJ Miller and RN Lea). 
Publications: Lockington (1880) (original description); Phillips (1966) (SW of Point Santa Cruz); Phillips (1967) (west of Moss Landing).

\section{Family HEXAGRAMMIDAE - greenlings}

Hexagrammos decagrammus (Pallas, 1810). Kelp Greenling. Museum specimens: MLMLF0830 (0.25 miles east of Hwy 1 Bridge at Moss Landing, B Antrim); CAS-SU 4147 (Monterey, DS Jordan); CAS-SU 58375 (off Yankee Point, JC Briggs).

Hexagrammos lagocephalus (Pallas, 1810). Rock Greenling. Museum specimens: CAS-ICH 21233 (Moss Beach, J Quast); LACM 3109 (N San Simeon, B Walker); UMMZ 63675 (Point Joe, CL and LC Hubbs).

Ophiodon elongatus Girard, 1854. Lingcod. Museum specimens: MLMLF0836 (north of Sandholdt Road Bridge at Moss Landing, LT Ackerman); SIO 62-269 (off Pigeon Point, EA Best); SIO 67-108 (Monterey Bay, CL Hubbs); SIO 84-46 (off Point Sur, W Wakefield). Publication: Yoklavich et al. (1991) (Hwy 1 Bridge and Dairy at Elkhorn Slough).

Oxylebius pictus Gill, 1862. Painted Greenling. Museum specimens: CAS-ICH 19581 (Soberanes Point tidepool, RR Harry); CAS-ICH 21234 (Monterey, WI Follett); CAS-ICH 39456 (Monastery Beach, L Hallacher).

Pleurogrammus monopterygius (Pallas, 1810). Atka Mackerel. Museum specimens: CAS-ICH 25733 (off Santa Cruz, identifier unknown); CAS-SU 47173 (off Monterey, RL Bolin); Publication: Bolin (1952) (outer coast of Monterey Peninsula).

Zaniolepis frenata Eigenmann and Eigenmann, 1889. Shortspine Combfish. Museum specimens: CAS-ICH 40333 (SSW of Point Piedras Blancas, WC Ruark); CASICH 46371 (Monterey Bay, M Hearne); USNM 148501.5223298 (west of Cambria, identifier unknown, "Albatross" Expedition).

Zaniolepis latipinnis Girard, 1858. Longspine Combfish. Museum specimens: CAS-ICH 12497 (off Point Montara, EC Johnston); CAS-ICH 42561 (Monterey Bay, RN Lea); CAS-SU 16001 (Monterey Bay, RL Bolin).

\section{Family RHAMPHOCOTTIDAE - grunt sculpins}

Rhamphocottus richardsonii Günther, 1874. Grunt Sculpin. Museum specimens: MLMLF0854 (Cannery Row, ME Anderson); SIO 00-49 (off Point San Simeon, RN Lea); SIO 01-79 (off Point Sur, RN Lea). 


\section{Family COTTIDAE - sculpins}

Artedius corallinus (Hubbs, 1926). Coralline Sculpin. Museum specimens: CASICH 39853 (off Lovers Point, RN Lea); CAS-ICH 51861 (south of Point Sur, RN Lea); CAS-SU 35364 (Point Lobos, RL Bolin).

Artedius fenestralis Jordan and Gilbert, 1883. Padded Sculpin. Museum specimens: CAS-ICH 21726 and CAS-ICH 21914 (off Alameda, San Francisco Bay, JD Hopkirk); SIO 07-185 (near Alcatraz, San Francisco Bay, RN Lea). Other records occur to the north of MBNMS, including CAS-ICH 27315 (Duxbury Point, HB Dietrich). Specimens south of San Francisco Bay were re-examined. Yankee Point specimen (KU 12802) re-determined as Clinocottus analis by William Leo Smith (University of Kansas, 26 Oct 2017). Diablo Canyon specimens (LACM 31700-004) re-determined as Artedius harringtoni (orbital cirrus present) by Rick Feeney (Natural History Museum Los Angeles County, Oct 2017). Southern-most records are from San Francisco Bay. We include here, because they presumably traversed MBNMS waters from the north.

Artedius harringtoni (Starks, 1896). Scalyhead Sculpin. Museum specimens: CASICH 41715 (Natural Bridges State Park, KM Howe); MLMLF0862 (main channel Elkhorn Slough, ME Anderson); CAS-SU 40884 (Point Lobos tidepool, RL Bolin).

Artedius lateralis (Girard, 1854). Smoothhead Sculpin. Described from Monterey and San Luis Obispo. Museum specimen: Syntype (location of Monterey specimen unknown); MLMLF0864 and MLMLF0865 (Carmel Point tidepools, E Yarberry); MLMLF0866 (off Davenport, J Gates and R Banek). Publication: Girard (1854) (original description).

Artedius notospilotus Girard, 1856. Bonyhead Sculpin. Museum specimens: CASICH 50403, 50411 (Moss Beach, D Begle); MLMLF0869 (Elkhorn Slough, B Antrim); CAS-SU 3852 (Monterey, RL Bolin).

Ascelichthys rhodorus Jordan and Gilbert, 1880. Rosylip Sculpin. Museum specimens: CAS-ICH 17861 (Pillar Point, MG Bradbury); CAS-ICH 20237 (Moss Beach, RR Harry); CAS-SU 49539 (Moss Beach, RF Ford).

Chitonotus pugetensis (Steindachner, 1876). Roughback Sculpin. Museum specimens: CAS-ICH 7360 (Monterey Bay, RL Bolin); CAS-ICH 12531 (west of Montara, EC Johnston); CAS-ICH 216330 (west of Santa Cruz, JD Hopkirk); MLMLF0881 (Kirby Park at Elkhorn Slough, ME Anderson).

Clinocottus acuticeps (Gilbert, 1986). Sharpnose Sculpin. Museum specimens: CAS-ICH 216269 (Moss Beach, RR Harry); CAS-ICH 225290 (Half Moon Bay, DA Neely); LACM 35299.003 (Half Moon Bay, C Swift and K Howe). 
Clinocottus analis (Girard, 1858). Wooly Sculpin. Described from Monterey. Museum specimens: Syntypes (FMNH 210, USNM 486); CAS-ICH 7371 (Point Lobos, RL Bolin); CAS-ICH 11964 (Moss Beach, RR Harry); CAS-ICH 32984 (north of San Simeon Beach, WN Eschmeyer and SG Poss). Publication: Girard (1858b) (original description).

Clinocottus embryum (Jordan \& Starks, 1895). Calico Sculpin. Museum specimens: CAS-ICH 78466 (Pigeon Point, D Catania); CAS-SU 35394 (Point Lobos, RL Bolin); CAS-SU 58467 (south of Yankee Point, JC Briggs).

Clinocottus globiceps (Girard, 1858). Mosshead Sculpin. Museum specimens: CASICH 25280 (Moss Beach, WI Follett and L Dempster); CAS-ICH 216419 (Soberanes Point, RL Bolin); CAS-SU 58415 (south of Yankee Point, JC Briggs).

Clinocottus recalvus (Greeley, 1899). Bald Sculpin. Described from Pacific Grove. Museum specimens: Holotype (CAS-SU 6068); CAS-ICH 25279 (Moss Beach, WI Follett and L Dempster); CAS-ICH 216412 (Soberanes Point, AO Flechsig); CAS-SU 16013 (Point Lobos, RL Bolin). Publication: Greeley (1899) (original description).

Enophrys bison (Girard, 1854). Buffalo Sculpin. Museum specimens: CAS-ICH 37545 (Moss Beach, RF Ford); MLMLF0903 (Hwy 1 Bridge at Elkhorn Slough, ME Anderson); CAS-SU 35329 (Del Monte Beach pier, RL Bolin).

Enophrys taurina Gilbert, 1914. Bull Sculpin. Described from Monterey Bay, near Pacific Grove. Museum specimens: Holotype (USNM 75064); CAS-ICH 66760 (off Otter Point to Lovers Point, RN Lea); CAS-ICH 232701 (Monterey Bay, TN Fast). Publication: Gilbert (1914) (original description).

Hemilepidotus hemilepidotus (Tilesius, 1811). Red Irish Lord. Museum specimens: CAS-ICH 35284 (Mussel Point, RL Bolin); SIO 72-86 (north of Point San Simeon, R Rosenblatt); UMMZ 63379 (Carmel Bay, CL Hubbs and LC Hubbs).

Hemilepidotus spinosus Ayres, 1854. Brown Irish Lord. Museum specimens: CASICH 18855 (Moss Beach, RR Harry); CAS-ICH 28471 (Carmel Bay, RN Lea); CASICH 36410 (Monterey, JE McCosker).

Icelinus burchami Evermann and Goldsborough, 1907. Dusky Sculpin. Museum specimens: CAS-ICH 234831 (off Point Sur, RL Bolin and PL Budd); SIO 01-117 (Carmel Canyon, P Reilly); SIO 99-80 (Monterey Bay, P Reilly and J Spratt).

Icelinus cavifrons Gilbert, 1890. Pit-head Sculpin. Museum specimens: LACM 52245.014 (off of San Simeon Point, E Hobson et al.); LACM 52277.008 and CASSU 64410 (Monterey Bay, TN Fast). 
Icelinus filamentosus Gilbert, 1890. Threadfin Sculpin. Museum specimens: CASICH 37505 (off Davenport, WN Eschmeyer); CAS-ICH 37518 (west of Point Sur, KR Hakanson); CAS-ICH 42547 (off Cypress Point, RN Lea); CAS-SU 23070 (Point Lobos, RL Bolin).

Icelinus fimbriatus Gilbert, 1890. Fringed Sculpin. Museum specimens: CAS-ICH 16145 (off Point Pinos, identifier unknown); CAS-SU 40922 (Monterey Bay, A Peden); CAS-SU 58419 (Monterey Bay, JC Briggs).

Icelinus oculatus Gilbert, 1890. Frogmouth Sculpin. Museum specimens: SIO $97-$ 126 (off Cypress Point, J Field); SIO 99-41 (off Point Sur, RN Lea); SIO 99-81 (Monterey Bay, RN Lea).

Icelinus quadriseriatus Lockington, 1880. Yellowchin Sculpin. Museum specimens: CAS-ICH 7364 (Monterey Bay, RL Bolin); CAS-ICH 12780 (off Point Montara, identifier unknown, US Fish Commission); CAS-SU 40925 (off Santa Cruz, RL Bolin).

Icelinus tenuis Gilbert, 1890. Spotfin Sculpin. Museum specimens: CAS-ICH 37520 (west of Point Sur, KR Hakanson); CAS-SU 21348 (Monterey Bay, RL Bolin); CASSU 58456 (Monterey Bay, JC Briggs).

Jordania zonope Starks, 1895. Longfin Sculpin. Museum specimens: LACM 52248.018 (Jade Cove, E Hobson); SIO 72-86 (north of Point San Simeon, R Rosenblatt); CAS-SU 35331 (off Asilomar, RL Bolin).

Leptocottus armatus Girard, 1854. Pacific Staghorn Sculpin. Museum specimens: CAS-ICH 48200 (Monterey Bay, S Richardson); CAS-SU 15028 (upper Elkhorn Slough, CL Hubbs); CAS-SU 47497 (Elkhorn Slough, WC Freihofer). Publication: Yoklavich et al. (1991) (Elkhorn Slough).

Oligocottus maculosus Girard, 1856. Tidepool Sculpin. Museum specimens: CASICH 18832 (Moss Beach: RR Harry); CAS-ICH 25276 (Moss Beach, WI Follett and L Dempster); CAS-SU 4244 (Pacific Grove, identifier unknown).

Oligocottus rimensis (Greeley, 1899). Saddleback Sculpin. Described from Point Lobos. Museum specimens: Holotype (CAS-SU 6067); CAS-ICH 47384 (Carmel kelp bed, RN Lea); CAS-ICH 48465 (Pigeon Point tidepools, D Catania); CAS-ICH 212726 (Soberanes Point tidepool, RR Harry). Publication: Greeley (1899) (original description).

Oligocottus rubellio (Greeley, 1899). Rosy Sculpin. Described from Monterey Bay. Museum specimens: Holotype (CAS-SU 6066); CAS-ICH 212628 (Soberanes Point, 
S Weitzman); CAS-SU 35342 (Point Lobos, RL Bolin); CAS-SU 40944 (Pescadero Point, RL Bolin); CAS-SU 48926 (north of San Simeon, M Bradbury). Publication: Greeley (1899) (original description).

Oligocottus snyderi Greeley, 1898. Fluffy Sculpin. Described from Pacific Grove. Museum specimens: Lectotype (CAS-SU 5846, Lectotype apparently established by Greeley 1899); Paralectotypes [CAS-SU 5847 (5)]; CAS-ICH 7361 (Point Lobos, RL Bolin); CAS-ICH 18628 (Moss Beach, RR Harry); CAS-ICH 27637 (Natural Bridges State Park, B Wesemann). Publications: Greeley in Jordan and Evermann (1898) (type specimen); Greeley (1899) (lectotype established).

Orthonopias triacis Starks and Mann, 1911. Snubnose Sculpin. Museum specimens: CAS-ICH 15793 (Carmel Bay, RN Lea); CAS-ICH 51862 (south of Point Sur, RN Lea); CAS-ICH 56947 (off Lovers Point, RN Lea). Publication: Gilbert (1914) (Monterey Bay).

Radulinus asprellus Gilbert, 1890. Slim Sculpin. Museum specimens: CAS-ICH 37496 (off Half Moon Bay, T Iwamoto); SIO 60-430 (south of San Simeon Point, W Dahlstrom); SIO 98-113 (Monterey Bay, RN Lea).

Radulinus boleoides Gilbert, 1898. Darter Sculpin. Museum specimen: CAS-ICH 40889 (SW of Soberanes Point, RN Lea); MLMLF0951 (Soberanes Point, B Antrim).

Ruscarius creaseri (Hubbs, 1926). Roughcheek Sculpin. Museum specimens: CASICH 30460 (Monterey Breakwater, ME Anderson); LACM 52248.005 (Jade Cove, ES Hobson); SIO 72-86 (off San Simeon Point, R Rosenblatt).

Scorpaenichthys marmoratus (Ayres, 1854). Cabezon. Museum specimens: CASICH 7365 (Elkhorn Slough, Bolin); CAS-ICH 11037 (Moss Beach, BW Halstead); CAS-ICH 32990 (north of San Simeon Beach State Park, WN Eschmeyer and SG Poss). Publication: Yoklavich et al. (1991) (Hwy 1 Bridge, Dairy, and Kirby Park at Elkhorn Slough).

Synchirus gilli Bean, 1890. Manacled Sculpin. Museum specimens: CAS-ICH 47391 (Carmel kelp bed, RN Lea); CAS-ICH 216550 (Carmel Bay kelp canopy, ME Anderson); CAS-SU 15521 (Monterey, RL Bolin).

Zesticelus profundorum (Gilbert, 1896). Flabby Sculpin. Museum specimens: MLMLF0967 (Monterey Bay, identifier unknown); SIO 85-69 (off Point Sur, W Wakefield); CAS-SU 25277 (off Point Pinos, RL Bolin, "Albatross" collection). Publication: Gilbert (1915) (Monterey, "Albatross" collection). 


\section{Family HEMITRIPTERIDAE - searavens}

Blepsias cirrhosus (Pallas, 1814). Silverspotted Sculpin. Museum specimens: LACM 47917.001 (San Simeon, BW Walker, AO Flechsig, AB Rechnitzer); SIO 50-193A (San Simeon Bay, F Taylor and AA Allanson); SIO 73-220 (Point Piedras Blancas, R Rosenblatt).

Nautichthys oculofasciatus (Girard, 1858). Sailfin Sculpin. Museum specimens: CAS-ICH 25890 and CAS-ICH 31066 (Pacific Grove, WI Follett); LACM 7939 (off San Simeon, B Walker); LACM 52248.025 (Jade Cove, ES Hobson).

\section{Family AGONIDAE - poachers}

Agonomalus mozinoi Wilimovsky and Wilson, 1979. Kelp Poacher. Museum specimens: Paratype (CAS-ICH 40716, Monastery Beach); CAS-ICH 64652 (off Cannery Row, Monterey, ME Anderson); SIO 09-291 (Carmel Bay, RN Lea). Publication: Wilimovsky and Wilson (1979) (original description). Recognized by some researchers as Hypsagonus mozinoi. We follow Sheiko and Mecklenburg (2004), and current usage in "Catalog of Fishes" (Eschmeyer et al. 2017), with placement in Agonomalus.

Agonopsis sterletus (Gilbert, 1898). Southern Spearnose Poacher. Museum specimen: UCLA W 66-67 (San Simeon Point, identifier unknown; specimen pending accession at SIO, out on loan to Russia at time of manuscript preparation). Another specimen (SIO 94-123) collected from nearby Morro Bay; therefore, locality is probable.

Agonopsis vulsa (Jordan \& Gilbert, 1880). Northern Spearnose Poacher. Museum specimens: MLMLF0975 (off Waddell Creek, GE Kukowski); MLMLF0976 (Monterey Bay, D Varoujean); CAS-SU 63622 (Monterey Bay, ME Anderson).

Bathyagonus pentacanthus (Gilbert, 1890). Bigeye Poacher. Museum specimens: SIO 91-115 (Monterey Bay, N Lai and J Butler); CAS-SU 16708 (off Point Sur, H Freeman); CAS-SU 64401 (off Pescadero Point, RL Bolin).

Bothragonus swanii (Steindachner, 1876). Rockhead. Museum specimens: CASICH 16152 (Pacific Grove, L Dempster); CAS-ICH 23946 (Carmel Bay, MG Bradbury); LACM 52245.006 (NW of San Simeon Point, E Hobson).

Chesnonia verrucosa (Lockington, 1880). Warty Poacher. Museum specimens: UCLA W 59-135 (4.5 miles west of Point Montara, identifier unknown; specimen pending accession at SIO, pers. comm. HJ Walker, Oct 2017); USNM 48731.5269927 (off Muir Beach, identifier unknown, "Albatross" Expedition). 
Odontopyxis trispinosa Lockington, 1880. Pygmy Poacher. Museum specimens: CAS-ICH 12347 (off Point Montara Light, EC Johnston); CAS-ICH 18651 (Pacific Grove, RR Harry); SIO 50-193A (San Simeon Bay, F Taylor and AA Allanson, SIO).

Stellerina xyosterna (Jordan \& Gilbert, 1880). Pricklebreast Poacher. Described from Santa Cruz beach at Monterey Bay. Museum specimens: Holotype [USNM (not found)]; other material [USNM 27173 and USNM 27395 (Soquel during 1880, DS Jordan)]; CAS-ICH 216557 (San Simeon Bay, AO Flechsig); MLMLF0995 (off Pajaro River at Monterey Bay, GE Kukowski). Publication: Jordan and Gilbert (1880g) (original description).

Xeneretmus latifrons (Gilbert, 1890). Blacktip Poacher. Museum specimens: CASICH 37497 (SSW of Half Moon Bay, T Iwamoto); LACM 30233.002 (off Cypress Point, W Dahlstrom); CAS-SU 3650 (west of Santa Cruz, H Freeman).

Xeneretmus leiops Gilbert, 1915. Smootheye Poacher. Museum specimens: SIO 19119 (Monterey Bay, RN Lea); SIO 97-122 (Carmel Bay, J Spratt and K Schlining); SIO 99-41 (off Point Sur, P Reilly, J Spratt and RN Lea).

Xeneretmus triacanthus (Gilbert, 1890). Bluespotted Poacher. Museum specimens: CAS-ICH 14270 (Monterey Bay, RL Bolin); SIO 60-430 (off San Simeon Point, W Wakefield); CAS-SU 69025 (Santa Cruz, A Peden).

\section{Family PSYCHROLUTIDAE - fathead sculpins}

Psychrolutes phrictus Stein and Bond, 1878. Blob Sculpin. Museum specimens: CAS-ICH 40740 (Santa Cruz, B Antrim); MLMLF0971 (off Santa Cruz, B. Antrim). Publications: Burton and Lundsten (2008), and Lundsten et al. (2009) (Davidson Seamount, based on imagery). Visual records: MBARI/NOAA T951-01 (Davidson Seamount: EJ Burton and L Lundsten).

\section{Family LIPARIDAE - snailfishes}

Bathyphasma ovigerum Gilbert, 1896. Abyssal Snailfish. Publication and Visual record: Stein et al. (2006) (Monterey Canyon). Previously recognized as Careproctus ovigerus. We follow naming convention of Balushkin (2012), and current usage in Catalog of Fishes (Eschmeyer et al. 2017). Common name follows Mecklenburg et al. (2002).

Careproctus filamentosus Stein, 1978. snailfish. Publication and Visual record: Stein et al. 2006 (Monterey Canyon). No official common name. 
Careproctus gilberti Burke, 1912. Smalldisk Snailfish. Museum specimen and Publication: CAS-ICH 29948 (Monterey Bay, Anderson et al. 1979).

Careproctus kamikawai Orr, 2012. Arbiter Snailfish. Described from Monterey Bay. Museum specimen: Holotype [USNM 400885 (ex. UW 150324)]. Visual records: MBARI/NOAA T425-08, MBARI/NOAA T425-09 and MBARI/NOAA T425-10 (Davidson Seamount, L Kuhnz). Publication: Orr (2012) (original description). Common name follows Orr (2012).

Careproctus longifilis Garman, 1892. Threadfin Snailfish. Museum specimen and Publication: MBARI 2000321-HFSS11 (Monterey Canyon, Stein et al. 2006).

Careproctus melanurus Gilbert, 1892. Blacktail Snailfish. Museum specimens: CAS-ICH 55146 (west of Pigeon Point, ME Anderson); CAS-ICH 55399 (off Point Sur, ME Anderson); SIO 97-127 (Carmel Canyon, J Field); SIO 99-81 (Monterey Bay, RN Lea). Publication and Visual record: Stein et al. (2006) (Monterey Canyon).

Elassodiscus caudatus (Gilbert, 1915). Humpback Snailfish. Described from Monterey Bay. Museum specimens: Holotype (USNM 75815); CAS-ICH 29947 (Monterey Bay, E Anderson). Publications: Gilbert (1915) (original description); Anderson et al. (1979) (Monterey Bay, CAS-ICH 29947).

Liparis florae (Jordan \& Starks, 1895). Tidepool Snailfish. Museum specimens: CAS-ICH 11649 (Moss Beach, RR Harry); CAS-ICH 19877 (Soberanes Point tidepool, RR Harry); CAS-ICH 47388 (Carmel kelp bed, RN Lea); CAS-ICH 30765 (north of San Simeon, AO Flechsig).

Liparis fucensis Gilbert, 1896. Slipskin Snailfish. Museum specimens: CAS-ICH 45980 (Monterey, RL Bolin); LACM 7904 (off San Simeon, B Walker); LACM 52248.02 (Jade Cove, E Hobson).

Liparis mucosus Ayres, 1855. Slimy Snailfish. Museum specimens: CAS-ICH 15044 (Pigeon Point, RN Lea); CAS-ICH 59630 (Carmel Bay, RN Lea); SIO 67-298 (off Piedras Blancas Lighthouse, D Wilkie and CJ Farwell); SIO 87-99 (Soberanes Point, RN Lea).

Liparis pulchellus Ayres, 1855. Showy Snailfish. Museum specimen: MLMLF1022 (off Waddell Creek, GE Kukowski); CAS-SU 16021 (Monterey Bay, RL Bolin).

Lipariscus nanus Gilbert, 1915. Pygmy Snailfish. Described from Monterey Bay. Museum specimens: Holotype (USNM 75817); Paratypes [CAS-SU 22993 (off Santa Cruz Lighthouse), CAS-SU 229994 (off Point Pinos Lighthouse)]; MLMLF1024 (Monterey Bay, ME Anderson). Publication: Gilbert (1915) (original description). 
Nectoliparis pelagicus Gilbert and Burke, 1912. Tadpole Snailfish. Museum specimens: MBARI V3-13-90 (Monterey Canyon, Stein et al. 2006); CAS-SU 63860 (Monterey Bay, RL Bolin); CAS-SU 63904 (Monterey Bay, TN Fast). Publications: Gilbert (1915) (Monterey Bay); Stein et al. (2006) (Monterey Canyon, MBARI V3-13-90).

Osteodiscus cascadiae Stein, 1978. Bonydisk Snailfish. Museum specimen and Publication: MBARI 2001276-HFSS6 (Monterey Canyon, Stein et al. 2006). Common name follows Love et al. (2005).

Paraliparis albescens Gilbert, 1915. Phantom Snailfish. Described from off Point Pinos. Museum specimens: Holotype (USNM 75816); CAS-ICH 29950 (Monterey Bay, ME Anderson); CAS-ICH 35952 (Monterey Canyon, ME Anderson); MLMLF1030 (Monterey Canyon, ME Anderson). Publications: Gilbert (1915) (original description); Anderson et al. (1979) (Monterey Bay/Canyon, CAS-ICH 29950, CASICH 35952, and MLMLF1030).

Paraliparis cephalus Gilbert, 1892. Swellhead Snailfish. Museum specimens: CASSU 5232 (SW of Santa Cruz, identifier unknown, US Fish Commission); CAS-SU 5254 (west of Point Año Nuevo, identifier unknown, US Fish Commission).

Paraliparis dactylosus Gilbert, 1896. Polydactyl Snailfish. Described from "off Santa Cruz" (station 3112, west of Point Año Nuevo). Museum specimens: Lectotype (USNM 48616, Lectotype established by Burke 1930); Paralectotypes [CAS-SU 3024 (1, west of Point Año Nuevo), USNM 53032 (1, west of Point Año Nuevo)]; MBARI 2002130-HFSS10 (Soquel Canyon; Stein et al. 2006). Publications: Gilbert (1896) (original description); Burke (1930); Stein et al. (2006) (Soquel Canyon, MBARI 2002130-HFSS10). Common name follows Mecklenburg et al. (2002).

Paraliparis deani Burke, 1912. Prickly Snailfish. Museum specimen: CAS-SU 22963 (Point Pinos, identifier unknown, US Fish Commission). Publication: Gilbert (1915) (Monterey Bay, US Fish Commission).

Paraliparis mento Gilbert, 1892. Bulldog Snailfish. Museum specimen: CAS-SU 22995 (Point Pinos, identifier unknown, US Fish Commission). Publication: Gilbert (1915) (Monterey Bay, US Fish Commission).

Paraliparis pectoralis Stein, 1978. Pectoral Snailfish. Museum specimen: SIO 9292 (off Monterey Bay, R Dotson, NMFS). Common name follows Mecklenburg et al. (2002).

Paraliparis rosaceus Gilbert, 1890. Rosy Snailfish. Museum specimens: CAS-ICH 31496 (Monterey Bay, ME Anderson); CAS-ICH 34960 (off Davenport, BS Antrim); MLMLF1034 (Ascension Canyon, ME Anderson). 
Paraliparis ulochir Gilbert, 1896. Broadfin Snailfish. Museum specimen: CAS-SU 23000 (Point Pinos, identifier unknown, US Fish Commission).

Rhinoliparis attenuatus Burke, 1912. Slim Snailfish. Museum specimens: CAS-SU 22960 and CAS-SU 22969 (off Point Pinos Lighthouse, identifier unknown, US Fish Commission). Publication: Gilbert (1915) (Monterey Bay, US Fish Commission).

Rhinoliparis barbulifer Gilbert, 1896. Longnose Snailfish. Museum specimens: MBARI 2002130-HFSS8 (Soquel Canyon, Stein et al. 2006); SIO 06-15 (east of Sur Ridge, KA Moots). Publication: Stein et al. (2006) (Soquel Canyon, MBARI 2002130-HFSS8).

\section{Order Perciformes}

Family MORONIDAE - temperate basses

Morone saxatilis (Walbaum, 1791). Striped Bass. Museum specimens: MLMLF1038 (Moss Landing Harbor mouth, LT Ackerman); SIO 54-76 (San Simeon Bay, A Rechnitzer); USNM 84567 (Monterey Bay, identifier unknown, "Albatross" Expedition). Publication: Yoklavich et al. (1991) (Hudson's Landing at Elkhorn Slough). Introduced from New Jersey into San Francisco Bay (1879 and 1882, Stevens and Kohlhorst 2001).

\section{Family POLYPRIONIDAE - wreckfishes}

Stereolepis gigas Ayres, 1859. Giant Sea Bass. Museum specimens: CAS-ICH 58785 (Monterey Bay during Jul 1986, RN Lea); MLMLF1039 (Monterey Bay during Aug 1978, El Niño, D Bedford). Publications: Phillips (1930b) (off Pacific Grove during 1930, El Niño); Phillips (1954) (off Pacific Grove during 1953). Categorized as occurring during warm-water events (e.g., El Niño).

\section{Family EPINEPHELIDAE - groupers}

Hyporthodus niphobles (Gilbert \& Starks, 1897). Star-studded Grouper. Museum specimens: LACM 38417.001 (off Point Piedras Blancas during Sept 1975, D Burge); SIO 95-24 (west of Hurricane Point during Nov 1994, RN Lea). Additional specimen captured west of Bixby Bridge during Oct 1992 and examined by RN Lea; providence unknown. This is a southern species that can move northward during warm-water events. Categorized as occurring during warm-water events (e.g., El Niño).

Mycteroperca xenarcha Jordan, 1888. Broomtail Grouper. Museum specimen: CASICH 58469 (Monterey Bay, RN Lea). 


\section{Family SERRANIDAE - sea basses and groupers}

Paralabrax clathratus (Girard, 1854). Kelp Bass. Museum specimens: MLMLF1049 (Cannery Row kelp beds during Nov 1973, El Niño, ME Anderson); CAS-SU 5556 (Pacific Grove, EC Starks, date unknown); USNM 110287 (Monterey, DS Jordan, date unknown, skull). Publications: Miller and Gotshall (1965) (Princeton west jetty during Sept 1960, after the warm water period, 1957-1959, small individuals appeared in skiff catch throughout Monterey Bay, larger fish landed from Santa Cruz to Monterey); Smith and Gotshall (1967) (Half Moon Bay during 1959). Categorized as occurring during warm-water events (e.g., El Niño).

Paralabrax maculatofasciatus (Steindachner, 1868). Spotted Sand Bass. Listed with reservation; possibly an historic record and/or occurring during warm-water events (e.g., El Niño). Publications: Boulenger (1895); Miller and Gotshall (1965); Miller and Lea (1972).

Miller and Lea (1972) reported the northern limit at Monterey and noted a record from San Francisco in the late 1800s. The 1800s record references Boulenger (1895, British Museum) who described the distribution of P. maculatofasciatus as "Coast of California and Mexico, from San Francisco to Mazatlan.” Boulenger (1895) listed three young specimens from San Francisco. However, no specimen currently exists at the British Natural History Museum (BMNH, Mar 2017) as indicated in 1895 publication. We assume these three young specimens were originally misidentified and subsequently removed from the collection. In addition, Jordan and Evermann (1896a, b) did not recognize the San Francisco record, and considered San Pedro as the northern limit (as did Jordan and Gilbert 1882, and Jordan and Eigenmann 1890).

The northern limit at Monterey, noted by Miller and Lea (1972), was likely in reference to Miller and Gotshall (1965) who suggested a range extension for P. maculatofasciatus per a sportfish party boat capture in Monterey during Feb 1963 (El Niño occurred during 1957-1959). The specimen was confirmed by Dan Miller (California Department of Fish and Game biologist), but not accessioned in a museum.

Paralabrax nebulifer (Girard, 1854). Barred Sand Bass. Described from Monterey. Museum specimens: Syntypes [USNM 282 (2)]. Publication: Girard (1854) (original description).

\section{Family PRIACANTHIDAE - bigeyes}

Pristigenys serrula (Gilbert, 1891). Popeye Catalufa. Museum specimens: CAS-ICH 52604 (off Point San Pedro, San Mateo County during 1983, El Niño, identifier unknown); CAS-ICH 54924 (off Davenport during 1983, El Niño, W Starnes). Publication: Starnes (1988) (Monterey Bay during 1983, El Niño). Categorized as occurring during warm-water events (e.g., El Niño). 


\section{Family MALACANTHIDAE - tilefishes}

Caulolatilus princeps (Jenyns, 1840). Ocean Whitefish. Publications: Miller and Gotshall (1965); Miller and Lea (1972). Typically occurs in warmer water; however, can move north during El Niño years. During 1957-1961, Miller and Gotshall (1965) reported Ocean Whitefish catches as far north as San Francisco. Miller and Lea (1972) list the species as occurring as far north as Vancouver Island, British Columbia; common in southern California, rare north of Monterey. Categorized as occurring during warm-water events (e.g., El Niño).

\section{Family CORYPHAENIDAE - dolphinfishes}

Coryphaena hippurus Linnaeus, 1758. Dolphinfish. Publication: Monterey County Herald (1997) (off Point Pinos). Fishermen caught two specimens off Point Pinos during 1997 El Niño; RN Lea confirmed catch (Monterey County Herald, 2 Sept 1997). Typically occurs in warmer water; however, can move north during El Niño years. Categorized as occurring during warm-water events (e.g., El Niño).

\section{Family ECHENEIDAE - remoras}

Remora australis (Bennett, 1840). Whalesucker. Museum specimen: CAS-ICH 26766 (off Santa Cruz, identifier unknown).

Remora remora (Linnaeus, 1758). Remora. Museum specimens: CAS-ICH 37868 (Monterey Bay, C Woodhill); SIO 93-197 (Monterey Bay, identifier unknown); UMMZ 64123 (off Santa Cruz, identifier unknown).

\section{Family CARANGIDAE - jacks}

Caranx caballus Günther, 1868. Green Jack. Museum specimen and Publication: CAS-ICH 54926 (Sea Cliff State Beach, Aptos during 1983, El Niño, Lea and Walker 1995). Categorized as occurring during warm-water events (e.g., El Niño).

Decapterus muroadsi (Temminck \& Schlegel, 1844). Amberstripe Scad. Museum specimens: CAS-SU 58626 (Pacific Grove, WF Smith-Vaniz); CAS-SU 68836 (Monterey Bay, JR Rainey). Publication: Frey (1962) (Monterey Bay, CAS-SU 58626).

Naucrates ductor (Linnaeus, 1758). Pilotfish. Museum specimens: CAS-ICH 54922 (3 miles south of Cape San Martin, RN Lea); CAS-SU 15483 (Mussel Point, Monterey, H Miller). 
Seriola dorsalis (Gill, 1863). Yellowtail Jack. Museum specimen: CAS-ICH 37523 (Monterey during 1976, WN Eschmeyer). Previously recognized as Seriola lalandi. We follow naming convention of Martinez-Takeshita et al. (2015), and current usage in Catalog of Fishes (Eschmeyer et al. 2017).

Trachurus symmetricus (Ayres, 1855). Jack Mackerel. Museum specimens: CASICH 7559 (Santa Cruz, B Halstead); CAS-ICH 66526 (Elkhorn Slough, DT Anderson); CAS-SU 48289 (Monterey Beach at Municipal Wharf, FH Berry); CAS-SU 58448 (Elkhorn Slough, JC Briggs). Publication: Fitch (1949) (Davidson Seamount).

\section{Family BRAMIDAE - pomfrets}

Brama japonica Hilgendorf, 1878. Pacific Pomfret. Museum specimen: CAS-ICH 39597 (Sea Cliff State Beach, Aptos, RN Lea).

Pteraclis aesticola (Jordan \& Snyder, 1901). Pacific Fanfish. Museum specimen: CAS-ICH 64200 (Sur Canyon during Aug 1987, RN Lea).

\section{Family CARISTIIDAE - manefishes}

Caristius macropus (Bellotti, 1903). Bigmouth Manefish. Museum specimen: SIO 98-117 (Canyon off Davenport during 1998, RN Lea).

\section{Family HAEMULIDAE - grunts}

Haemulon californiensis (Steindachner, 1876). Salema. Museum specimens: UW 3172 (4, San Francisco Bay region during 1932, C. Wade). Publication: Phillips (1936) (southern Monterey Bay, 1935). During Mar 2017, RN Lea examined 4 museum specimens and new x-rays (UW 3172), and confirmed identifications. This is a southern species that can move northward during warm-water events. Categorized as historic and occurring during warm-water events (e.g., El Niño).

\section{Family POLYNEMIDAE - threadfins}

Polydactylus approximans (Lay \& Bennett, 1839). Blue Bobo. Museum specimen: CAS-SU 35305 (Monterey Bay during 1941, warm-water event, RL Bolin). Publications: Follett (1948) (Monterey during Jan 1941, CAS-SU 35305); Lea and Rosenblatt (2000) (references Follett 1948 and museum specimen). Categorized as occurring during warm-water events (e.g., El Niño). 


\section{Family SCIAENIDAE - drums and croakers}

Atractoscion nobilis (Ayres, 1860). White Seabass. Publication: Radovich (1961) (Monterey during 1958 and 1959, El Niño). Categorized as occurring during warmwater events (e.g., El Niño).

Genyonemus lineatus (Ayres, 1855). White Croaker. Museum specimens: CAS-ICH 19866 (Pacific Grove, DA Simpson); CAS-ICH 66670 (Monterey Bay, MG Bradbury); MLMLF1086 (near Sandholdt Road Bridge at Moss Landing, LT Ackerman). Publication: Phillips (1936) (southern Monterey Bay).

Menticirrhus undulatus (Girard, 1854). California Corbina. Listed with reservation; possibly an historic record and/or occurring during warm-water events (e.g., El Niño). Publication: Starks (1919). In a review of the croakers (Sciaenidae) of California, Starks (1919) noted "This fish is rather common on sandy shores of southern California and is known southward into the Gulf of California, while individuals are sometimes taken as far northward as San Francisco." Stark's information may be based on CAS-SU 21232, from San Francisco, collected by Charles H. Gilbert. No date is listed, but it was likely taken in the late 1800 s to early 1900 s based on catalog number. We know of one recent record from north of Point Conception, a $474 \mathrm{~mm}$ TL fish caught off Morro Rock in Jan 1986 (CAS-ICH 58470); south of MBNMS. During warm-water events, occurrence of this species needs to be verified off the sandy beaches of central California.

Seriphus politus Ayres, 1860. Queenfish. Museum specimens: ANSP 11543 (San Francisco before 1880, identifier unknown, US Fish Commission); MLMLF1092 (Kirby Park at Elkhorn Slough, E Yarberry); CAS-SU 4153 (San Francisco Market, DS Jordan). Publications: Ayres (1860b) (San Francisco); Phillips (1932a) (Monterey Bay during 1931, El Niño); Skogsberg (1939) (Monterey Bay); Yoklavich et al. (1991) (Kirby Park and Hudson's Landing at Elkhorn Slough). Occurs in the coastal waters of the Northeast Pacific (Miller and Lea 1972). We include the San Francisco specimens here, because they presumably traversed MBNMS waters.

\section{Family KYPHOSIDAE - sea chubs}

Girella nigricans (Ayres, 1860). Opaleye. Museum specimens: CAS-ICH 66680 (cove SE of Pigeon Point, identifier unknown, 1963); CAS-ICH 76622 (Mussel Point, Pacific Grove, C Limbaugh, Mead, and Patterson); CAS-SU 12083 (Pacific Grove, EC Starks). Publications: Limbaugh (1955) (Pacific Grove); Norris (1963) (Monterey).

Hermosilla azurea Jenkins and Evermann, 1889. Zebraperch. Publication: Phillips (1965a) (Monterey). Visual record: SIMoN (2018) (Monterey Harbor during Apr 2012, C King). Based on molecular phylogenetic analysis, recent publications propose a taxonomic revision of the family Kyphosidae, including a name change 
from Hermosilla azurea to Kyphosus azureus (Knudsen and Clements 2013, 2016). We do not adopt the name change here and await further widespread acceptance of this proposed revision.

Kyphosus vaigiensis (Quoy \& Gaimard, 1825). Blue-bronze Chub. Museum specimen: CAS-ICH 56945 (Monterey Bay, Coast Guard Breakwater during 1984, El Niño, RN Lea, as Kyphosus analogus). Categorized as occurring during warm-water events (e.g., El Niño). Knudsen and Clements (2013) determined Kyphosus analogus (Gill, 1862) a junior synonym of K. vaigiensis (Quoy \& Gaimard, 1825).

Medialuna californiensis (Steindachner, 1876). Halfmoon. Museum specimens: CAS-SU 11871 (Monterey Bay during Sept 1896, SM Duarte); CAS-SU 35303 (vicinity of Half Moon Bay during Sept 1941, warm water year, RL Bolin); CAS-SU 35304 (off Point Sur, RL Bolin, date unknown). Publication: Radovich (1961) (Santa Cruz Municipal Wharf during 1958, El Niño). Categorized as occurring during warmwater events (e.g., El Niño).

\section{Family PENTACEROTIDAE - armorheads}

Pseudopentaceros wheeleri (Hardy, 1983). North Pacific Armorhead. Museum specimens: CAS-ICH 26759 (off Pigeon Point during 1960, WI Follett, as Pentaceros richardsoni); LACM 45682.001 (north of Piedras Blancas during 1991, moderate El Niño, identifier unknown, as Pentaceros pectoralis). North Pacific species is Pseudopentaceros wheeleri and includes records of Pentaceros richardsoni and Pseudopentaceros pectoralis (Humphreys et al. 1989, Love et al. 2005).

\section{Family OPLEGNATHIDAE - knifejaws}

Oplegnathus fasciatus (Temminck \& Schlegel, 1844). Barred Knifejaw. Publication and Visual records: Ta et al. (2018) (southern Monterey Bay). Ta et al. (2018) reported the first records of the Western Pacific Ocean Barred Knifejaw in the Northeast Pacific Ocean, including at least two individual knifejaws at multiple sites in southern Monterey Bay between Dec 2014 and Sept 2015 (Del Monte Beach, San Carlos Beach, McAbee Pinnacle, and South Breakwater wall off Monterey Harbor entrance). These observations, along with others in Oregon and Washington, were spatially and temporally correlated with the arrival of Japanese tsunami marine debris. Additional observations by lead author Ta, of the assumedly lone fish, occurred between Oct and Dec 2018 (Monterey Herald, 10 Dec 2018). The Barred Knifejaw is native to Japan, Korea, and China in warm-temperate to tropical seas. These specimens observed from 
central California to Washington are unlikely to reproduce due to warmer temperature requirements; however, they can continue to grow in cold water. Transported with tsunami debris from the Western Pacific Ocean. Common name follows Ta et al. (2018).

\section{Family EMBIOTOCIDAE - surfperches}

Amphistichus argenteus Agassiz, 1854. Barred Surfperch. Museum specimens: MLMLF1108 (Moss Landing Beach, D Varoujean); CAS-SU 33423 (Waddell Creek, WI Follett); CAS-SU 58374 (Carmel Beach, JC Briggs).

Amphistichus koelzi (Hubbs, 1933). Calico Surfperch. Museum specimens: MLMLF1110 (Marina Beach at Monterey Bay, D Varoujean); CAS-SU 5364 (San Simeon Bay, identified by "Gsm" likely George S. Myers, US Fish Commission); CAS-SU 34296 (Half Moon Bay, WI Follett).

Amphistichus rhodoterus (Agassiz, 1854). Redtail Surfperch. Museum specimens: CAS-ICH 26972 (Muir Beach, Marin County, WI Follett); CAS-ICH (Sunset State Beach, K Oda, specimen pending accession at CAS, RN Lea, May 2018); MLMLF1111 (south jetty at Moss Landing, ME Anderson); CAS-SU 34289 (Half Moon Bay, WI Follett).

Brachyistius frenatus Gill, 1862. Kelp Perch. Museum specimens: CAS-ICH 2191 (Stillwater Cove, identifier unknown); MLMLF1113 (off Sandholdt Pier at Moss Landing at Monterey Bay, L Talent); CAS-SU 58412 (Hopkins Marine Station, JC Briggs).

Cymatogaster aggregata Gibbons, 1854. Shiner Perch. Museum specimens: CASICH 25476 (San Simeon, CL Hubbs, LC Hubbs, WI Follett et al.); CAS-SU 49531 (Moss Beach, RF Ford); CAS-SU 51276 (Elkhorn Slough, D Cohen). Publication: Yoklavich et al. (1991) (Elkhorn Slough).

Embiotoca caryi Agassiz, 1853. Rainbow Seaperch. Museum specimens: CASICH 18204 (Elkhorn Slough, M Morten); CAS-ICH 25426 (Santa Cruz Wharf, WI Follett); CAS-SU 58379 (Hopkins Marine Station, JC Briggs). Previously recognized as Hypsurus caryi (Agassiz, 1853). We follow naming convention of Longo et al. (2018).

Embiotoca jacksoni Agassiz, 1853. Black Perch. Museum specimens: MLMLF1117 (Kirby Park at Elkhorn Slough, E Yarberry); CAS-SU 22477 (Pacific Grove, John O. Snyder); CAS-SU 47496 (Elkhorn Slough, WC Freihofer). Publication: Yoklavich et al. (1991) (Hwy 1 Bridge, Dairy, and Kirby Park at Elkhorn Slough). 
Embiotoca lateralis Agassiz, 1854. Striped Seaperch. Museum specimens: CAS-SU 16783 (Martin's Beach, south Half Moon Bay, GS Myers); CAS-SU 25783 (Moss Beach, WI Follett); CAS-SU 58427 (south of Carmel, JC Briggs).

Hyperprosopon argenteum Gibbons, 1854. Walleye Surfperch. Museum specimens: CAS-SU 58388 (Carmel Beach, JC Briggs); CAS-SU 58389 (Hopkins Marine Station, JC Briggs); CAS-SU 58447 (Elkhorn Slough, JC Briggs). Publication: Yoklavich et al. (1991) (Hwy 1 Bridge, Dairy, Kirby Park, and Hudson's Landing at Elkhorn Slough).

Hyperprosopon ellipticum (Gibbons, 1854). Silver Surfperch. Museum specimens: MLMLF1123 (off Skipper's dock at Elkhorn Slough, B Antrim); CAS-SU 34294 (Half Moon Bay, WI Follett); CAS-SU 58386 (Carmel Beach, JC Briggs).

Hypocritichthys analis (Agassiz, 1861). Spotfin Surfperch. Museum specimens: CAS-ICH 25471 (San Simeon, CL Hubbs, LC Hunns, WI Follett et al.); CAS-ICH 27641 (Natural Bridges State Park, Santa Cruz, RR Rofen et al.); CAS-SU 34299 (Princeton Pier at Half Moon Bay, WI Follett). Previously recognized as Hyperprosopon anale Agassiz, 1861. We follow naming convention of Longo et al. (2018).

Micrometrus aurora (Jordan \& Gilbert, 1880). Reef Perch. Described from Monterey Bay; obtained from San Francisco market. Museum specimens: Syntypes [numerous specimens, including: ANSP 9272 (1), USNM 26996 (16)]; CAS-ICH 27642 (Natural Bridges State Park, Santa Cruz, RR Rofen et al.); CAS-ICH 212354 (Carmel Beach, BW Walker, A Flechsig); CAS-SU 48890 (east of Point Piedras Blancas, M Bradbury). Publication: Jordan and Gilbert (1880j) (original description).

Micrometrus minimus (Gibbons, 1854). Dwarf Perch. Museum specimens: CASSU 15995 (Monterey Bay, K Stanton); CAS-SU 19316 (Elkhorn Slough, D Cohen); CAS-SU 58411 (Hopkins Marine Station, JC Briggs). Publication: Yoklavich et al. (1991) (Hwy 1 Bridge and Dairy at Elkhorn Slough).

Phanerodon atripes (Jordan \& Gilbert, 1880). Sharpnose Seaperch. Described from Monterey Bay and Santa Cruz; obtained from San Francisco market. Museum specimens: Syntypes [numerous specimens, including: ANSP 9203 (1), USNM 26987 (orig. 80, now 7)]; CAS-ICH 51865 (Big Sur, RN Lea). Publications: Jordan and Gilbert (1880k) (original description); Lea (1972) (Monterey area).

Phanerodon furcatus Girard, 1854. White Seaperch. Museum specimens: CAS-ICH 20377 (San Simeon, CL Hubbs, LC Hubbs, WI Follett et al.); CAS-ICH 25427 (Santa Cruz, WI Follett); CAS-SU 51269 (Elkhorn Slough, WC Freihofer). Publication: Yoklavich et al. (1991) (Hwy 1 Bridge, Dairy, and Kirby Park at Elkhorn Slough).

Phanerodon vacca (Girard, 1855). Pile Perch. Museum specimens: LACM 4370 (Moss Beach, BW Halstead); LACM 8252 (San Simeon Point, BW Walker); CAS-SU 
58393 (Carmel Beach, JC Briggs); UMMZ 142380 (Elkhorn Slough, R Bolin and CL Hubbs). Publication: Yoklavich et al. (1991) (Hwy 1 Bridge, Dairy, and Kirby Park at Elkhorn Slough). Previously recognized as Damalichthys vacca Girard, 1855. We follow naming convention of Longo et al. (2018).

Rhacochilus toxotes Agassiz, 1854. Rubberlip Seaperch. Museum specimens: LACM 52230.008 (off San Simeon Point, JE Bleck); MLMLF1136 (Kirby Park at Elkhorn Slough, B Antrim); CAS-SU 12034 (Pacific Grove, EC Starks). Publication: Yoklavich et al (1991) (Hwy 1 Bridge and Dairy at Elkhorn Slough).

Zalembius rosaceus (Jordan and Gilbert, 1880). Pink Seaperch. Museum specimens: CAS-ICH 37178 (off Santa Cruz, T Iwamoto); CAS-ICH 37530 (west of Point Año Nuevo, KR Hakanson); CAS-SU 19135 (Monterey Bay, D Cohen).

\section{Family POMACENTRIDAE - damselfishes}

Chromis punctipinnis (Cooper, 1863). Blacksmith. Museum specimens: MLMLF1148 (Monastery Beach during Nov 1970, E Starks); CAS-SU 15997 (Monterey Bay during 1937, RL Bolin). Publications: Starks (1919) (Monterey Bay during 1918); Phillips (1961) (Carmel Bay during 1959, El Niño); Radovich (1961) (Monterey Bay during 1959, El Niño). Categorized as occurring during warm-water events (e.g., El Niño).

Hypsypops rubicundus (Girard 1854). Garibaldi. Described from Monterey. Museum specimens: Syntypes [MCZ 14825 (1, San Diego), USNM 484 (2, San Diego)]. Publications: Girard (1854) (original description); Girard (1858a) (2 adults from "Monterey, Cal.", refers to type specimens). Original description (and Girard 1858a) states "From Monterey, Cal."; however, only specimens from San Diego apparently exist (USNM 484). Observations are extremely rare in MBNMS; no other museum specimens are known. During Jun 2016 and Feb 2017, a single juvenile Garibaldi was observed in Monterey Bay Aquarium's (MBA) outdoor Great Tide Pool (MBA video imagery, pers. comm. Andrew Morgan, MBA Dive Officer/Exhibit Dive Coordinator). We cannot rule out the possibility the Garibaldi specimen was released from the aquarium kelp tank by way of the filtration system (i.e., as larvae). We categorize the listing here as Historic, and possibly occurring during warm-water events (e.g., El Niño).

\section{Family LABRIDAE - wrasses}

Oxyjulis californica Günther, 1861. Señorita. Described from Monterey. Museum specimens: Syntypes [USNM 706-707 (2,1)]; LACM 4922 (San Simeon, ES Hobson); MLMLF1164 (off Skipper's Dock at Elkhorn Slough, ME Anderson); CAS-SU 3895 (Pacific Grove, JO Snyder); CAS-SU 4156 (Monterey, CH Gilbert). Publication: Günther (1861) (original description). 
Semicossyphus pulcher (Ayres, 1854). California Sheephead. Museum specimen: USNM 110755 (Monterey, DS Jordan, date unknown). Publications: Phillips (1932a) (Monterey Bay during 1931, El Nińo); Radovich (1961) (Point Pinos during 1958, El Niño). Categorized as occurring during warm-water events (e.g., El Niño).

\section{Family BATHYMASTERIDAE - ronquils}

Rathbunella alleni Gilbert, 1904. Stripefin Ronquil. Described from Monterey Bay. Museum specimens: Holotype (CAS-SU 8415); Paratype [CAS-SU 8416 (Monterey Bay)]; CAS-ICH 57636 (Monterey Bay, AC Matarese); CAS-SU 23016 (Pacific Grove, AC Matarese). Publications: Gilbert (1904) (original description); Stevenson and Matarese (2005) (Monterey Bay and other).

Ronquilus jordani (Gilbert, 1889). Northern Ronquil. Museum specimens: MLMLF1169 (Monterey Bay, G McDonald); OS 7632 (off Monterey Bay, J Cobb); CASSU 36071 (Monterey Bay, J Waler and RL Bolin).

\section{Family ZOARCIDAE - eelpouts}

Bothrocara brunneum (Bean, 1890). Twoline Eelpout. Museum specimens: CASICH 25990 (off Pescadero Point, ME Anderson); CAS-ICH 47395 (Monterey Canyon, RN Lea); CAS-ICH 55411 (off Point Sur, ME Anderson). Publication: Burton and Lundsten (2008) (Davidson Seamount, based on imagery). Visual records: MBARI/NOAA T427-02, MBARI/NOAA D943-07, and MBARI/NOAA T946-05 (Davidson Seamount, EJ Burton, L Kuhnz, and L Lundsten).

Bothrocara molle Bean, 1890. Soft Eelpout. Museum specimen: CAS-ICH 60291 (west of Point Año Nuevo, ME Anderson); UW 46821 (east of Sur Ridge, RC Harrison); UW 113723 (Smooth Ridge at Monterey Bay, JR Hoff).

Eucryphycus californicus (Starks \& Mann, 1911). Persimmon Eelpout. Museum specimens: CAS-ICH 17623 (Monterey Canyon, GM Cailliet and RN Lea); CASICH 38671 (Monterey Bay, RG Kliever). Publication: Cailliet and Lea (1977) (Monterey Bay).

Lycenchelys callista Anderson, 1995. eelpout. Described from off Point Sur. Museum specimens: Holotype (CAS-ICH 55412); Paratypes [CAS-ICH 55062 (2, off Point Sur, ME Anderson), CAS-ICH 80630 (29, off Point Sur, ME Anderson); SIO 84-249 (21, off Point Sur), SIO 85-51 (22, off Point Sur)]. Publication: Anderson (1995) (original description). No official common name. 
Lycenchelys camchatica (Gilbert \& Burke, 1912). Kamchatka Eelpout. Museum specimens: CAS-ICH 31495 (Monterey Canyon, ME Anderson); CAS-ICH 55406 and CAS-ICH 56234 (off Point Sur, ME Anderson). Publication: Anderson et al. (1979) (Monterey Canyon, CAS-ICH 31495).

Lycenchelys crotalinus (Gilbert, 1890). Snakehead Eelpout. Museum specimens: CAS-ICH 55063 and CAS-ICH 55407 (off Point Sur, ME Anderson); CAS-ICH 57467 (Monterey Canyon, ME Anderson). Previously recognized as Embryx crotalinus. We follow Anderson (1995), Anderson and Fedorov (2004), and current usage in Catalog of Fishes (Eschmeyer et al. 2017); with placement in Lycenchelys.

Lycenchelys jordani (Evermann \& Goldsborough, 1907). Shortjaw Eelpout. Museum specimen: CAS-ICH 78979 (Point Sur, ME Anderson).

Lycenchelys micropora Andriashev, 1955. Manytoothed Eelpout. Museum specimens: CAS-ICH 234380 (off Monterey Bay, ME Anderson); UW 40727 (off Point Sur, W Wakefield and ME Anderson). Common name follows Mecklenburg et al. (2002).

Lycenchelys monstrosa Anderson, 1982. eelpout. Museum specimen: CAS-ICH 233977 (off Monterey Bay, ME Anderson). No official common name.

Lycodapus dermatinus Gilbert, 1896. Looseskin Eelpout. Museum specimens: CASICH 31493 (Monterey Canyon, ME Anderson); CAS-ICH 55403 (off Point Sur, ME Anderson); MLMLF1187 (off Point Sur, ME Anderson).

Lycodapus fierasfer Gilbert, 1890. Blackmouth Eelpout. Museum specimens: CASICH 35989 (Monterey Canyon, ME Anderson); CAS-ICH 58480 (SW of Point Sur, RN Lea); CAS-ICH 88443 (Monterey Bay, G Cailliet). Publication: Burton and Lundsten (2008) (Davidson Seamount, based on imagery). Visual record: MBARI/ NOAA T426-05 (Davidson Seamount, L Kuhnz).

Lycodapus mandibularis Gilbert, 1915. Pallid Eelpout. Described from Monterey Bay. Museum specimens: Holotype (USNM 75823); Paratypes [CAS-SU 22990 (1, Monterey Bay), CAS-SU 25765 (5, now 4, Monterey Bay); USNM 149514 (2, Monterey Bay)]. Publications: Gilbert (1915) (original description); Burton and Lundsten (2008) (Davidson Seamount, based on imagery). Visual record: MBARI/NOAA T946-01 (Davidson Seamount, L Lundsten).

Lycodapus pachysoma Peden and Anderson, 1981. Stout Eelpout. Museum specimen: UW 47335 (within Davidson Seamount Management Zone, J Hoff). Common name follows Mecklenburg et al. (2002). 
Lycodapus psarostomatus Peden and Anderson, 1981. Specklemouth Eelpout. Museum specimen: CAS-ICH 58902 (Point Pinos, ME Anderson). Common name follows Mecklenburg et al. (2002).

Lycodes cortezianus (Gilbert, 1890). Bigfin Eelpout. Museum specimens: CAS-ICH 37485 (west of Santa Cruz, T Iwamoto); CAS-ICH 39892 (west of Point Lobos, WC Ruark); MLMLF1171 (Monterey Bay, ME Anderson).

Lycodes diapterus Gilbert, 1892. Black Eelpout. Museum specimens: CAS-ICH 29299 and CAS-ICH 29945 (Monterey Bay, ME Anderson); CAS-ICH 37489 (Santa Cruz, T Iwamoto).

Lycodes pacificus (Collett, 1879). Blackbelly Eelpout. Museum specimens: CASICH 28873 and CAS-ICH 30881 (Monterey Bay, ME Anderson); USNM 227179 (Monterey, ME Anderson).

Lyconema barbatum Gilbert, 1896. Bearded Eelpout. Described from outside Monterey Bay. Museum specimens: Lectotype (USNM 48582, Lectotype established in Jordan and Evermann 1900, and traced to USNM 48582 by Springer and Anderson 1997); Paralectotypes including [CAS-SU 3627 (1), CAS-SU 69673 (4), USNM 53036 (1)]. Publications: Gilbert (1896) (original description); Jordan and Everman (1900) (lectotype established); Springer and Anderson (1997).

Melanostigma pammelas Gilbert, 1896. Midwater Eelpout. Described from Monterey Bay. Museum specimens: Holotype (USNM 53034, missing); Paratypes [USNM 48599 (nearly disintegrated), USNM 53034, CAS-SU 4000]; MLMLF1212 (Monterey Bay, ME Anderson). Publications: Gilbert (1896) (original description); Gilbert (1915) (Monterey Bay).

Pachycara bulbiceps (Garman, 1899). Snubnose Eelpout. Publications: Anderson and Peden (1988) (off Queen Charlotte Islands, British Columbia, to the Gulf of Panama, North Pacific, at depths of 2,601 to 4,000 m); Burton and Lundsten (2008), and Lundsten et al. (2009) (Davidson Seamount, based on imagery). Visual records: MBARI/NOAA D947-05 (Davidson Seamount, Jacobson Stout et al. 2017); MBARI D990-02 and MBARI D438-05 (Monterey Canyon, Jacobson Stout et al. 2017). Common name follows Mecklenburg et al. (2002).

Pachycara karenae Anderson, 2012. eelpout. Described from Monterey Bay. Museum specimens: Holotype (CAS-ICH 233971); Paratypes (CAS-ICH 233972, USNM 405384). Publication: Anderson (2012) (original description). No official common name. 


\section{Family STICHAEIDAE - pricklebacks}

Anoplarchus purpurescens Gill, 1861. High Cockscomb. Museum specimens: CASICH 7507 (Moss Beach, RR Harry); CAS-ICH 25196 (Carmel Bay, WI Follett); CAS-ICH 232759 (Point Lobos, RL Bolin); CAS-ICH 32985 (north of San Simeon Beach, WN Eschmeyer and SG Poss).

Cebidichthys violaceus (Girard, 1854). Monkeyface Prickleback. Museum specimens: CAS-ICH 18297 (Moss Beach, CL Hubbs); CAS-ICH 18662 (Pacific Grove, RR Harry); CAS-ICH 33004 (north of San Simeon, WN Eschmeyer and SG Poss); CAS-SU 47498 (Elkhorn Slough, WC Freihoffer).

Chirolophis nugator (Jordan \& Williams, 1895). Mosshead Warbonnet. Museum specimens: CAS-ICH 25893 (Pacific Grove, WI Follett); LACM 6608.005 (off San Simeon, ES Hobson); LACM 52248.012 (Jade Cove, ES Hobson); CAS-SU 64363 (Point Lobos tidepool, RL Bolin).

Ernogrammus walkeri Follett and Powell, 1988. Masked Prickleback. Described from west of San Simeon Point. Museum specimens: Holotype (CAS-ICH 56198); numerous Paratypes including [CAS-ICH 27156 (Monterey), CAS-ICH 48094 (off Pacific Grove), CAS-ICH 57410 (US Coast Guard breakwater, Monterey), CAS-ICH 57635 (Monterey Bay), SIO 72-86 (north of San Simeon Point)]. Publication: Follett and Powell (1988) (original description).

Esselenichthys carli (Follett \& Anderson, 1990). Threeline Prickleback. Described from Santa Barbara (holotype), Monterey, and south to Baja California. Museum specimens: Paratype (CAS-ICH 25892); SIO 53-193 (Pacific Grove, WI Follett). Publication: Follett and Anderson (1990) (original publication).

Kasatkia seigeli Posner and Lavenberg, 1999. Sixspot Prickleback. Museum specimen: SIO 03-92 (off Pacific Grove at Monterey Bay, RN Lea).

Phytichthys chirus (Jordan \& Gilbert, 1880). Ribbon Prickleback. Described from Point Pinos, Pacific Grove. Museum specimens: Lectotype (USNM 27175, Lectotype selected by Springer and Anderson 1997); Paralectotypes [USNM 200384 (1), 336453 (2)]; CAS-SU 48291 (Soberanes Point, RL Bolin); CAS-SU 48301 (Soberanes Point, WC Freihofer). Publications: Jordan and Gilbert (1880e) (original description); Springer and Anderson (1997).

Plagiogrammus hopkinsii Bean, 1894. Crisscross Prickleback. Described from Monterey Bay. Museum specimens: Holotype (USNM 44721); CAS-ICH 54642 (Pescadero Point, RL Bolin); CAS-SU 21199 (Pacific Grove, JO Snyder); CAS-SU 35302 (Point Lobos, RL Bolin and WE Ripley). Publication: Bean (1894) (original description). 
Plectobranchus evides Gilbert, 1890. Bluebarred Prickleback. Museum specimen and Publication: USNM 77460 (off Point Pinos, "Albatross" Explorations on the California Coast during 1904, Gilbert 1915).

Xiphister atropurpureus (Kittlitz, 1858). Black Prickleback. Museum specimens: CAS-ICH 14266 (between Yankee and Soberanes Points, RL Bolin and Marr); CASSU 16037 (Monterey Bay tidepool, A Calhoun); CAS-SU 58371 (south of Yankee Point, JC Briggs).

Xiphister mucosus (Girard, 1858). Rock Prickleback. Museum specimens: CASICH 18705 (Carmel Bay, RR Harry); CAS-ICH 18842 (Moss Beach, RR Harry); CAS-SU 16038 (Monterey Bay tidepool, A Calhoun); CAS-SU 48902 (east of Point Piedras Blancas, M Bradbury).

\section{Family PHOLIDAE - gunnels}

Apodichthys flavidus Girard, 1854. Penpoint Gunnel. Museum specimens: CASICH 11948 (Moss Beach, RR Harry); CAS-ICH 25894 (Pacific Grove, WI Follett); MLMLF1240 (off Skipper's Dock at Elkhorn Slough, ME Anderson); CAS-SU 47505 (Monterey, WC Freihofer); CAS-SU 58470 (Carmel Beach, JC Briggs).

Apodichthys fucorum. Jordan and Gilbert, 1880. Rockweed Gunnel. Described from Point Pinos. Museum specimens: Lectotype (USNM 26994, Lectotype selected by Springer and Anderson 1997); numerous Paralectotypes including [ANSP 10501 (Monterey); USNM 335151 (Monterey, 37)]; CAS-ICH 18693 (Moss Beach, RR Harry); CAS-ICH 26108 (Pacific Grove, WI Follett); CAS-ICH 47390 (Carmel kelp bed, RN Lea); CAS-ICH 32995 (north of San Simeon, WN Eschmeyer and SG Poss). Publication: Jordan and Gilbert (1880e) (original description).

Pholis clemensi Rosenblatt, 1964. Longfin Gunnel. Publication and Visual record: (Point Lobos, Pillar Point, Kline et al. 2013).

Pholis ornata (Girard, 1854). Saddleback Gunnel. Museum specimens: CAS-ICH 20238 and CAS-ICH 20239 (Moss Beach tidepool, identifier unknown); CAS-SU 63688 (Carmel Beach, RL Bolin).

Pholis schultzi Schultz, 1931. Red Gunnel. Museum specimens: LACM 6608.006 (off San Simeon Point, ES Hobson); LACM 7942 (off San Simeon Point, B Walker); SIO 72-86 (north of San Simeon Point, R Rosenblatt).

Ulvicola sanctaerosae Gilbert and Starks, 1897. Kelp Gunnel. Museum specimens: CAS-ICH 14989 (Pacific Grove, RN Lea); CAS-ICH 39860 (Carmel Bay, RN Lea); UCM 6639 (Half Moon Bay, TP Maslin). 


\section{Family ANARHICHADIDAE - wolffishes}

Anarrhichthys ocellatus Ayres, 1855. Wolf-eel. Museum specimens: ANSP 25047 (Pacific Grove, H Heath); CAS-ICH 31229 (Monterey Bay, WC Freihofer); CAS-SU 12610 (Pacific Grove, EC Starks).

\section{Family ZAPRORIDAE - prowfishes}

Zaprora silenus Jordan, 1896. Prowfish. Museum specimens: CAS-ICH 30693 (Monterey Bay, ME Anderson and GM Cailliet); CAS-ICH 47403 (between Bixby Creek and Carmel Bay, RN Lea); MLMLF1254 (Monterey Bay, GM Cailliet and ME Anderson). Publication: Cailliet and Anderson (1975) (Monterey Bay, CASICH 30693).

\section{Family SCYTALINIDAE - graveldivers}

Scytalina cerdale Jordan and Gilbert, 1880. Graveldiver. Museum specimens: CASICH 52435 (Moss Beach, D Catania); CAS-SU 58431 (south of Carmel, JC Briggs); UMMZ 36955 (Point Lobos, CL Hubbs).

\section{Family CHIASMODONTIDAE - swallowers}

Chiasmodon niger Johnson, 1864. Black Swallower. Museum specimens: UW 48697 (outside MBNMS, west of Ragged Point during Oct 1999, RN Clark, NMFS, $1034 \mathrm{~m}$, included here due to proximity); UW 48713 (west of Carmel during Oct 1999, RN Clark, NMFS, 1084 m). Publication: Lauth (2000) (UW specimens). Visual records: MBARI D241-06 and MBARI D0451-07 (Monterey Canyon, Jacobson Stout et al. 2017). Previously recognized as Chiasmodon subniger Garman, 1899. We follow naming convention of Prokofiev and Kukuev (2009), and Prokofiev (2014) where Chiasmodon subniger Garman, 1899 is considered a synonym of Chiasmodon niger Johnson, 1864.

Kali indica Lloyd, 1909. Pacific Sandfish. Museum specimen: UW 48679 (west of Carmel during Oct 1999, D Kamikawa, 1146 m).

\section{Family TRICHODONTIDAE - sandfishes}

Trichodon trichodon (Tilesius, 1813). Pacific Sandfish. Publication: Ayres 1860a. Trichodon lineatus Ayres 1860 is considered a synonym of Trichodon trichodon (Tilesius 1813). The type description of Trichodon lineatus by Ayres (1860) was based on 
one specimen from a "market in San Francisco." The holotype is presumably lost (Eschmeyer et al. 2017). The capture location is unknown; however, in 1860 captures were probably fairly close to San Francisco, and sold within 1 day of capture. Specimen likely occurred in, or traversed, MBNMS boundary. No other records found within MBNMS. Categorized as Historic.

\section{Family AMMODYTIDAE - sand lances}

Ammodytes hexapterus Pallas, 1814. Pacific Sand Lance. Museum specimens: CASICH 14284 (Rodeo Beach, Marin County, L Dempster); MLMLF1288 (Elkhorn Slough main channel between jetties, ME Anderson); MLMLF1290 (Monterey Bay, M Stevenson).

\section{Family URANOSCOPIDAE - stargazers}

Kathetostoma averruncus Jordan and Bollman, 1890. Smooth Stargazer. Museum specimens: CAS-ICH 56960 (Monterey Bay during 1984, GM Cailliet); CAS-ICH 57637 (Monterey Bay during 1985, RN Lea); LACM 32181.001 (off Point Piedras Blancas during 1960, L Pinkas); MLMLF1293 (off Soquel Point at Monterey Bay during 1984, GM Cailliet). Typically a southern species, museum specimens collected shortly after warm water events (i.e., 1983-1984, 1957-1959). Categorized as occurring during warm-water events (e.g., El Niño).

\section{Family BLENNIIDAE - combtooth blennies}

Hypsoblennius gentilis (Girard, 1854). Bay Blenny. Described from Monterey. Museum specimens: Holotype (USNM 489); CAS-ICH 13710 (Monterey Breakwater during 1970, CL Hubbs); CAS-ICH 243620 (Elkhorn Slough during 2016, G Longo); SIO 93-189 (Elkhorn Slough during 1993, RN Lea). Publication: Girard (1854) (original description).

Hypsoblennius gilberti (Jordan, 1882). Rockpool Blenny. Museum specimen: LACM 38727.001 (Carmel Bay during 1976, RN Lea).

Hypsoblennius jenkinsi (Jordan \& Evermann, 1896). Mussel Blenny. Museum specimens: CAS-ICH 56607 (Santa Cruz Municipal Wharf during 1985, G. McDonald, examined by RN Lea); CAS-ICH 242460 (Monterey Commercial Wharf during 2016, El Niño, G Longo). Publication: Nelson (1986) (Santa Cruz Municipal Wharf during 1985, CAS-ICH 56607). Typically a southern species, museum specimens collected shortly after warm water events (i.e., 1983-1984). Categorized as occurring during warm-water events (e.g., El Niño). 


\section{Family CLINIDAE - kelp blennies}

Gibbonsia elegans (Cooper, 1864). Spotted Kelpfish. Museum specimens: ANSP 16302 (Pacific Grove during 1897, El Niño, H Heath); CAS-ICH 2190 (Pebble Beach, Monterey County during 1928, RR Harry); LACM 9950.038 (San Simeon during 1963, BW Walker). Typically a southern species, south of Point Piedras Blancas (Miller and Lea 1972). Categorized as occurring during warm-water events (e.g., El Nińo).

Gibbonsia metzi Hubbs, 1927. Striped Kelpfish. Described from Pacific Grove. Museum specimens: Holotype (UMMZ 55004); CAS-ICH 7554 (Monterey, RR Harry); CAS-ICH 27644 (Natural Bridges State Park, E Byron); CAS-ICH 232453 (Soberanes Point, RN Lea); CAS-SU 15488 (Elkhorn Slough, CL Hubbs and RL Bolin). Publication: Hubbs (1927) (original description).

Gibbonsia montereyensis Hubbs, 1927. Crevice Kelpfish. Described from Pacific Grove. Museum specimens: Holotype (UMMZ 55003); CAS-ICH 25194 (Carmel Bay, WI Follett); CAS-ICH 37922 (NW of San Simeon, W Boyd); CAS-SU 48898 (1.75 miles east of Point Piedras Blancas, M Bradbury). Publication: Hubbs (1927) (original description).

Heterostichus rostratus Girard, 1854. Giant Kelpfish. Museum specimens: MLMLF1307 (east of Hwy 1 Bridge at Elkhorn Slough, B Antrim); CAS-SU 5555 (Pacific Grove, EC Starks); CAS-SU 15136 (Del Monte Beach, Monterey, CL Hubbs).

\section{Family CHAENOPSIDAE - tube blennies}

Neoclinus blanchardi Girard, 1858. Sarcastic Fringehead. Museum specimens: CAS-ICH 42556 (Carmel Bay, D Gotshall); MLMLF1314 (Elkhorn Slough between jetties, R Helm); CAS-SU 2288 (Pacific Grove, C Hubbs); CAS-SU 19183 (Monterey Bay, D Cohen and WC Freihofer).

Neoclinus stephensae Hubbs, 1953. Yellowfin Fringehead. Museum specimens: CAS-ICH 14403 (Monterey Harbor during 1964, DC Powell). Visual record: SIMoN (2018) (Monterey Harbor during Mar 2007 and 2010, El Niños, SI Lonhart). Categorized as occurring during warm-water events (e.g., El Niño).

Neoclinus uninotatus Hubbs, 1953. Onespot Fringehead. Museum specimens: Paratypes [Pacific Grove: CAS-ICH 7414, CAS-SU 2285, CAS-SU 23022]; CAS-ICH 26298 (Monterey, WI Follett). Publications: Hubbs (1953) (original description); Yoklavich et al. (1991) (Hwy 1 Bridge at Elkhorn Slough). 


\section{Family ICOSTEIDAE - ragfishes}

Icosteus aenigmaticus Lockington, 1880. Ragfish. Museum specimens: CAS-ICH 47399 (Monterey Bay, RN Lea); CAS-ICH 53094 (Santa Cruz Municipal Wharf during 1948, WI Follett); LACM 32682.001 (San Simeon Bay during 1972, identifier unknown); CAS-SU 58300 (off Point Sur during 1961, JB Phillips). Publications: Snyder (1913) (Pacific Grove); Thompson (1921b) (Monterey).

\section{Family GOBIESOCIDAE - clingfishes}

Gobiesox maeandricus (Girard, 1858). Northern Clingfish. Museum specimens: CAS-SU 1681 (Monterey, EC Starks); CAS-SU 15212 (Pacific Grove tidepool, JC Briggs); CAS-SU 58434 (south of Carmel, JC Briggs).

Rimicola muscarum (Meek \& Pierson, 1895). Kelp Clingfish. Described from Monterey Bay. Museum specimens: Holotype (CAS-SU 3030); Paratype [USNM 48875 (1, Monterey Bay)]; MLMLF0540 (Carmel Point tidepools, GE Kukowski); MLMLF0542 (Monterey Bay drift kelp, ME Anderson). Publication: Meek and Pierson (1895) (original description).

\section{Family GOBIIDAE - gobies}

Acanthogobius flavimanus (Temminck \& Schlegel, 1845). Yellowfin Goby. Museum specimens: MLMLF1328 (Elkhorn Slough, D Varoujean); MLMLF1329 (Kirby Park at Elkhorn Slough, D Varoujean); SIO 10-96 (Elkhorn Slough, RN Lea). Publication: Yoklavich et al. (1991) (station location from Barry 1983, Hudson's Landing at Elkhorn Slough). Species "inadvertently introduced from the Orient" (Miller and Lea 1972).

Clevelandia ios (Jordan \& Gilbert, 1882). Arrow Goby. Museum specimens: CASICH 19629 (Elkhorn Slough, CL Hubbs, RL Bolin et al.); CAS-SU 15029 (Elkhorn Slough, C Hubbs); CAS-SU 58450 (Elkhorn Slough, JC Briggs). Publication: Yoklavich et al. (1991) (Hwy 1 Bridge and Hudson's Landing at Elkhorn Slough).

Gillichthys mirabilis Cooper, 1864. Longjaw Mudsucker. Museum specimens: MLMLF1340 (near yacht club at Elkhorn Slough, E Yarberry); MLMLF1341 (Kirby Park at Elkhorn Slough, LT Ackerman); MLMLF1342 (Kirby Park at Elkhorn Slough, B Stewart). Publications: Cailliet et al. (1977) (larvae at Elkhorn Slough); Yoklavich et al. (1991) (station location from Barry 1983, Hudson's Landing at Elkhorn Slough).

Lepidogobius lepidus (Girard, 1858). Bay Goby. Museum specimens: CAS-ICH 56232 (Monterey Bay, ME Anderson); MLMLF1344 (Kirby Park at Elkhorn Slough, 
GE Kukowski). Publications: Yoklavich et al. (1991) (Hwy 1 Bridge, Dairy, and Kirby Park at Elkhorn Slough).

Lethops connectens Hubbs, 1926. Halfblind Goby. Described from Carmel Bay. Museum specimens: Holotype (UMMZ 63281); Paratypes [UMMZ 63282 (2)]; LACM 52277.01 (west of San Simeon Point, ES Hobson, J Bleck, T Chess, L Richards, J Kastendiek, A Harrington, J Morin). Publication: Hubbs (1926) (original description).

Lythrypnus dalli (Gilbert, 1890). Bluebanded Goby. Museum specimen: CAS-ICH 54911 (Monterey Coast Guard Breakwater during 1983, warm-water event, RN Lea). Categorized as occurring during warm-water events (e.g., El Niño).

Lythrypnus zebra (Gilbert, 1890). Zebra Goby. Museum specimen: CAS-ICH 31994 (Monastery Beach during 1975, ME Anderson).

Rhinogobiops nicholsii (Bean, 1882). Blackeye Goby. Museum specimens: MLMLF1350 (Monterey Breakwater, ME Anderson); MLMLF1352 (Elkhorn Slough, ME Anderson); CAS-SU 15116 (south of Malpaso Creek, C Hubbs).

Typhlogobius californiensis Steindachner, 1879. Blind Goby. Museum specimen: UCLA W 62-92 (cove north of San Simeon Point, identifier unknown, specimen pending accession at SIO, pers. comm. HJ Walker, Oct 2017).

\section{Family LUVARIDAE - louvars}

Luvarus imperialis Rafinesque, 1810. Louvar. Museum specimens: CAS-ICH 13245 (Santa Cruz during Sept 1945, WM Chapman); CAS-SU 14224 (Monterey Bay during Nov 1939, RL Bolin).

\section{Family SPHYRAENIDAE - barracudas}

Sphyraena argentea Girard, 1854. Pacific Barracuda. Museum specimen: USNM 109985 (Monterey, DS Jordan, date unknown). Publications: Phillips (1932a) (Monterey Bay during 1931, El Niño); Radovich (1961) (Monterey during 1958, El Niño). Common south of Morro Bay (Miller and Lea 1972). Categorized as occurring during warm-water events (e.g., El Niño).

\section{Family GEMPYLIDAE - snake mackerels}

Ruvettus pretiosus Cocco, 1833. Oilfish. Museum specimen: CAS-ICH 54919 (40 miles west of Monterey during 1983, J Hardwick). Typically occurs in warmer wa- 
ter; however, can move north during El Niño years. Categorized as occurring during warm-water events (e.g., El Niño).

\section{Family TRICHIURIDAE - cutlassfishes}

Aphanopus intermedius Parin, 1983. Intermediate Scabbardfish. Museum specimens (previously recognized as A. carbo): CAS-ICH 40254 (west of Pigeon Point, B Antrim and ME Anderson); CAS-ICH 42570 (off Pigeon Point, RN Lea); CAS-ICH 57643 (west of Santa Cruz, RN Lea). Common name follows Love et al. (2005).

Benthodesmus pacificus Parin and Becker, 1970. North Pacific Frostfish. Museum specimen: CAS-ICH 30692 (Moss Landing during 1968, ME Anderson).

Lepidopus fitchi Rosenblatt and Wilson, 1987. Pacific Scabbardfish. Museum specimen: CAS-ICH 67930 (off Montara during 1962, identifier unknown).

\section{Family SCOMBRIDAE - mackerels}

Auxis rochei (Risso, 1810). Bullet Mackerel. Museum specimen: CAS-ICH 56944 (between Half Moon Bay and San Francisco Bay during 1984, El Niño, RN Lea). Eastern Pacific population of $A$. rochei has been described as a subspecies (Auxis rochei eudorax Collette and Aadland, 1996), including record within MBNMS (CAS-ICH 56944). Typically occurs in warmer seas; however, can move north during El Niño years. Categorized as occurring during warm-water events (e.g., El Niño).

Katsuwonus pelamis (Linnaeus, 1758). Skipjack Tuna. Publications: Radovich (1961) (off Davidson Seamount); Lea (1997) (off Lopez Point). Found off Davidson Seamount (distance not specified) during 1957 El Niño (Radovich 1961). School observed surface feeding on Pacific sardine off Lopez Point on 25 Oct 1997; four captured (El Nińo, Lea 1997). Typically occurs in warmer seas; however, can move north during El Niño years. Categorized as occurring during warm-water events (e.g., El Niño). There are no museum specimens collected within MBNMS; however, large fish are less commonly stored in museums.

Sarda chiliensis (Cuvier, 1832). Pacific Bonito. Publications: Phillips (1932a) (Monterey Bay during 1931, El Nińo); Fast (1957) (Monterey Bay during 1956, warmwater event); Radovich (1961) (Monterey during 1959, El Niño). Categorized as occurring during warm-water events (e.g., El Nińo).

Scomber japonicus Houttuyn, 1782. Pacific Chub Mackerel. Museum specimen: UMMZ 176337 (Pacific Grove, 1959, El Niño, RM Bailey). Publications: Phillips 
(1932a) (Monterey Bay during 1931 El Nino); Phillips (1937) (near Pacific Grove). Categorized as occurring during warm-water events (e.g., El Niño).

Scomberomorus concolor (Lockington 1879). Gulf Sierra. Described from San Francisco Market, and "probably taken...in Monterey Bay." Museum specimens: Holotype (CAS-ICH lost specimen in 1906); USNM 27205 (Soquel, DS Jordan, date unknown). Publications: Lockington (1879) (original description); Phillips (1932b) (Monterey during 1931, El Niño). Categorized as occurring during warm-water events (e.g., El Niño).

Thunnus alalunga (Bonnaterre, 1788). Albacore. Museum specimen: USNM 270550 (San Simeon during 1941, identifier unknown). Publications: Andrews (1927) (off Point Sur during Sept 1925 and fall 1926, El Niño); Phillips (1931a) (SW of Point Pinos during 1930, El Niño); Phillips (1932a) (south of Monterey Bay during 1931, El Niño); Fitch (1949) (Davidson Seamount); Fast (1957) (Monterey Bay area during Sept 1956, warm-water event); Radovich (1961) (Monterey during 1931, El Niño); CDFW (2017) [Sport Fishing Record (Santa Cruz during 1997, El Niño)]. Categorized as occurring during warm-water events (e.g., El Niño).

Thunnus albacares (Bonnaterre, 1788). Yellowfin Tuna. Publication: Fast (1957) (Monterey Bay during 1956, warm-water event). Categorized as occurring during warm-water events (e.g., El Niño). There are no catalogued records collected within MBNMS; however, large fish are less commonly stored in museums.

Thunnus obesus (Lowe, 1839). Bigeye Tuna. Publication: Radovich (1961). Published range is Peru to Iron Springs, Washington; considered rare off California (Miller and Lea 1972). Considered a tropical species but occurs north during warm-water events. Radovich (1961) noted movement north during 1959 El Niño (off Cape Mendocino, CA and Iron Springs, WA). Categorized as occurring during warm-water events (e.g., El Niño). There are no catalogued records collected within MBNMS; however, large fish are less commonly stored in museums.

Thunnus orientalis (Temminck \& Schlegel, 1844). Pacific Bluefin Tuna. Museum specimen: CAS-ICH 213885 (as synonym Thunnus thynnus; between Point Pinos and Point Joe during 1958, El Niño, WI Follett). Publications: Fast (1957) (Monterey Bay during 1956, warm-water event); Radovich (1961) (Monterey during 1957, El Niño). Categorized as occurring during warm-water events (e.g., El Niño).

\section{Family XIPHIIDAE - swordfishes}

Xiphias gladius Linnaeus, 1758. Swordfish. Publication: Radovich (1961) (Monterey and Davidson Seamount area during 1957, El Nińo). Categorized as occurring during 
warm-water events (e.g., El Niño). There are no catalogued records collected within MBNMS; however, large fish are less commonly stored in museums.

\section{Family ISTIOPHORIDAE - billfishes}

Kajikia audax (Philippi, 1887). Striped Marlin. Publication: Hill and Haight (1985) (32 km SW of Santa Cruz during 1983, El Nińo, examined by RN Lea). Hill and Haight (1985) reported a striped marlin capture $32 \mathrm{~km}$ southwest of Santa Cruz by sport fisherman on 5 Sept 1983 (during an extensive El Niño event, specimen examined and identified by RN Lea). Categorized as occurring during warm-water events (e.g., El Nińo). There are no catalogued records collected within MBNMS; however, large fish are less commonly stored in museums.

\section{Family CENTROLOPHIDAE - medusafishes}

Icichthys lockingtoni Jordan and Gilbert, 1880. Medusafish. Museum specimens: CAS-ICH 37491 (Santa Cruz, T Iwamoto); CAS-ICH 218179 (Monterey Canyon, EH Ahlstrom); CAS-ICH 218182 (Pacific Grove, WI Follett); SWFSC uncatalogued (Davidson Seamount during May 2015, RN Lea and EJ Burton).

\section{Family TETRAGONURIDAE - squaretails}

Tetragonurus cuvieri Risso, 1810. Smalleye Squaretail. Museum specimens: CASICH 14272 (SW of Santa Cruz during 1959, El Niño, identifier unknown); LACM 9888.001 (off Big Sur during Oct 1967, K Mais, California Department of Fish and Game). Publications: Fitch (1949, 1951) (Davidson Seamount during 1948); Radovich (1961) (Monterey Bay during 1959, El Niño). Usually occurs in deep water, well offshore (Miller and Lea 1972). Categorized as occurring during warm-water events (e.g., El Niño).

\section{Family STROMATEIDAE - butterfishes}

Peprilus simillimus (Ayres, 1860). Pacific Pompano. Museum specimens: CAS-ICH 79889 (Monterey Bay, Freihofer); MLMLF1387 (Kirby Park at Elkhorn Slough, E Yarberry); MLMLF1388 (Moss Landing Harbor, E Yarberry); UMMZ 63402 (Monterey Bay, WL Scofield). 


\section{Order Pleuronectiformes \\ Family PARALICHTHYIDAE - sand flounders}

Citharichthys sordidus (Girard, 1854). Pacific Sanddab. Museum specimens: CASICH 238635 (Monterey Bay, RR Rofen); CAS-SU 23735 (Santa Cruz, D Villadolid); SWFSC uncatalogued (Davidson Seamount during May 2015, W Watson); UAM 730 (Elkhorn Slough, identifier unknown).

Citharichthys stigmaeus Jordan and Gilbert, 1882. Speckled Sanddab. Museum specimens: CAS-ICH 26296 (Monterey, WI Follett); MLMLF1397 (Kirby Park at Elkhorn Slough, LT Ackerman); CAS-SU 23688 (Pacific Grove, D Villadolid); SWFSC uncatalogued (Davidson Seamount during May 2015, W Watson). Publications: Yoklavich et al. (1991) (Hwy 1 Bridge and Dairy at Elkhorn Slough); Brown (2006) (Elkhorn Slough).

Citharichthys xanthostigma Gilbert, 1890. Longfin Sanddab. Museum specimens: CAS-ICH 228405 (Monterey Bay, R Bolin); NCSM 80806 (34, Monterey Bay, FJ Schwartz). Publication: Phillips (1967) (SSW of Point Santa Cruz).

Hippoglossina stomata Eigenmann and Eigenmann, 1890. Bigmouth Sole. Museum specimen: SIO 05-88 (Lucia Canyon, D Kamikawa). Publication: Phillips (1965b) (off Salinas River at Monterey Bay).

Paralichthys californicus (Ayres, 1859). California Halibut. Museum specimens: MLMLF1404 (Kirby Park at Elkhorn Slough, E Yarberry); MLMLF1405 (Kirby Park at Elkhorn Slough, LT Ackerman); CAS-SU 12176 (Pacific Grove, EC Starks). Publications: Phillips (1945) (off Moss Landing); Yoklavich et al. (1991) (Hwy 1 Bridge, Kirby Park, and Hudson's Landing at Elkhorn Slough).

Xystreurys liolepis Jordan and Gilbert, 1880. Fantail Sole. Museum specimen: MLMLF1406 (Monterey Bay during 1987, J Brennan); Publication: Phillips (1963) (off Salinas River at Monterey Bay during Jul 1962).

\section{Family PLEURONECTIDAE - righteye flounders}

Atheresthes evermanni Jordan and Starks, 1904. Kamchatka Flounder. Museum specimen and Publication: SIO 96-72 (off Davenport, Lea 2013).

Atheresthes stomias (Jordan \& Gilbert, 1880). Arrowtooth Flounder. Museum specimen: CAS-ICH 26232 (off Half Moon Bay during 1957, identifier unknown). 
Clidoderma asperrimum (Temminck \& Schlegel, 1846). Roughscale Sole. Museum specimen: SIO 95-23 (west of Half Moon Bay, RN Lea). Common name follows Lea et al. (1989).

Embassichthys bathybius (Gilbert, 1890). Deepsea Sole. Museum specimens: CASICH 5617 (off Point Sur, ME Anderson); CAS-ICH 15075 (off Montara, identifier unknown); CAS-ICH 34349 (Monterey Canyon, ME Anderson); CAS-ICH 42565 (off Cypress Point, RN Lea).

Eopsetta jordani (Lockington, 1879). Petrale Sole. Museum specimens: ANSP 16325 (Pacific Grove, Harold Heath); CAS-ICH 45961 (Point Montara, E Johnston and F Sumner, US Fish Commission); USNM 77420 (off Santa Cruz Lighthouse, identifier unknown, "Albatross" Explorations on the California Coast, 1904).

Glyptocephalus zachirus Lockington, 1879. Rex Sole. Museum specimens: CASICH 48187 (Monterey Bay, S Richardson); CAS-ICH 233952 (off Point Pinos, CL Hubbs); SIO 67-102 (within Davidson Seamount Management Zone, CL Hubbs).

Hippoglossoides elassodon Jordan and Gilbert, 1880. Flathead Sole. Museum specimens: MLMLF1420 (Monterey Bay, T Dimitre); SIO 11-32 (Monterey Bay, HC McWilliams, NMFS). Publication: Allen and Smith (1988) (survey data indicate southern boundary at Monterey).

Hippoglossus stenolepis Schmidt, 1904. Pacific Halibut. Publications: Starks (1919) (Monterey Bay); Phillips (1935) (Monterey); Phillips (1958) (4 miles south of Point Piedras Blancas during Sept 1957, a warm-water year).

Isopsetta isolepis (Lockington, 1880). Butter Sole. Museum specimens: CAS-ICH 27646 (Natural Bridges State Park, B Wesemann); CAS-ICH 39768 (Monterey Bay, RL Bolin).

Lepidopsetta bilineata (Ayres, 1855). Rock Sole. Museum specimens: CAS-ICH 40341 (NW of Point Pinos, T Iwamoto); MLMLF1422 (off Waddell Creek, E Yarberry); CAS-SU 3606 (off Point Piedras Blancas, identifier unknown, US Fish Commission). Publication: Gilbert (1915) (Monterey Bay).

Lyopsetta exilis (Jordan \& Gilbert, 1880). Slender Sole. Museum specimens: CASSU 16043 and CAS-SU 16044 (Monterey Bay, RL Bolin); CAS-SU 23698 (Monterey Bay, D Villadolid). Publication: Gilbert (1915) (Monterey Bay).

Microstomus pacificus (Lockington, 1879). Dover Sole. Museum specimens: CASICH 37533 (west of Point Año Nuevo, WN Eschmeyer); SIO 67-102 (within David- 
son Seamount Management Zone, CL Hubbs, 35 mm); CAS-SU 18680 (off Monterey Bay, RL Bolin); CAS-SU 25653 (Santa Cruz, EC Starks).

Parophrys vetulus Girard, 1854. English Sole. Museum specimens: CAS-ICH 26233 (off Point Montara, identifier unknown); CAS-SU 16047 (Monterey Bay, RL Bolin); CAS-SU 58452 (Elkhorn Slough, JC Briggs). Publications: Gilbert (1915) (Monterey Bay); Yoklavich et al. (1991) (Hwy 1 Bridge, Dairy, Kirby Park, and Hudson's Landing at Elkhorn Slough); Brown (2006) (Elkhorn Slough).

Platichthys stellatus (Pallas, 1787). Starry Flounder. Museum specimens: CAS-SU 12166 (Pacific Grove, RL Bolin); CAS-SU 16428 (Monterey Bay, RL Bolin); CASSU 34279 (Elkhorn Slough, WI Follett). Publication: Yoklavich et al (1991) (Hwy 1 Bridge, Dairy, Kirby Park, and Hudson's Landing at Elkhorn Slough).

Pleuronichthys coenosus Girard, 1854. C-O Sole. Museum specimen: CAS-SU 58398 (Monterey Bay, JC Briggs).

Pleuronichthys decurrens Jordan and Gilbert, 1881. Curlfin Turbot. Museum specimens: CAS-ICH 26081 (Half Moon Bay, identifier unknown); CAS-SU 3605 (offshore north of Cambria, identifier unknown, US Fish Commission); CAS-SU 51270 (Monterey Bay, WC Freihofer). Publication: Yoklavich et al. (1991) (Hwy 1 Bridge and Dairy at Elkhorn Slough).

Pleuronichthys guttulatus Girard, 1856. Diamond Turbot. Museum specimens: CAS-ICH 23877 (Sea Cliff State Park, WI Follett); CAS-SU 4426 (Monterey, identifier unknown); CAS-SU 58381 (Elkhorn Slough, JC Briggs). Publication: Yoklavich et al. (1991) (Dairy, Kirby Park, and Hudson's Landing at Elkhorn Slough).

Pleuronichthys verticalis Jordan and Gilbert, 1880. Hornyhead Turbot. Museum specimens: KU 1620 (Monterey, RL Bolin); SIO 85-155 (Pigeon Point, D Gibson); UF 79823 (Monterey Bay, G Burgess).

Psettichthys melanostictus Girard, 1854. Sand Sole. Museum specimens: MLMLF1445 (Moss Landing Harbor mouth, LT Ackerman); CAS-SU 4379 (Monterey, DS Jordan); CAS-SU 12164 (Pacific Grove, EC Starks); CAS-SU 58471 (Carmel Beach: JC Briggs). Publication: Gilbert (1915) (Monterey Bay).

\section{Family CYNOGLOSSIDAE - tonguefishes}

Symphurus atricaudus (Jordan \& Gilbert, 1880). California Tonguefish. Museum specimens: CAS-ICH 24050 (Monterey Bay, RR Harry); MLMLF1449 (Kirby Park at Elkhorn Slough, E Yarberry). Publications: Phillips (1942b) (Monterey area during 1942); Yoklavich et al. (1991) (Hwy 1 Bridge and Hudson's Landing at Elkhorn Slough). 


\section{Order Tetraodontiformes \\ Family BALISTIDAE - triggerfishes}

Balistes polylepis Steindachner, 1876. Finescale Triggerfish. Museum specimens: CAS-ICH 33303 (off Manresa State Beach during 1975, ME Anderson); CAS-ICH 50060 (off Hopkins Marine Station during 1982, D Powell); CAS-ICH 52482 (outside Pillar Point Harbor during 1983, El Nińo, L Dempster); CAS-SU 18100 (Monterey Harbor during Jun 1951, RL Bolin). Publications: Bolin (1952) (Monterey Harbor during Jun 1951); Radovich (1961) (Monterey Bay during 1958, El Niño). Categorized as occurring during warm-water events (e.g., El Niño).

\section{Family DIODONTIDAE - porcupinefishes}

Diodon holocanthus Linnaeus, 1758. Balloonfish. Museum specimen: CAS-SU 52706 (San Gregorio Beach during 1958, El Niño, JM Leis). Typically occurs in warm seas; rare off California (Miller and Lea 1972). Categorized as occurring during warmwater events (e.g., El Niño).

\section{Family MOLIDAE - molas}

Mola mola (Linnaeus, 1758). Ocean Sunfish. Museum specimens: LACM 55986.001 (Monterey, J O'Sullivan); MLMLF1457 (Monterey Bay, ME Anderson). Publication: Gotshall (1961) (Monterey Bay).

\section{Extralimital species}

The geographic ranges for the following 18 species encompass MBNMS boundaries. They are likely to occur within MBNMS; however, no verifiable records occur from within MBNMS. Until further evidence is found, we consider these extralimital species.

\section{Order Rajiformes \\ Family ARHYNCHOBATIDAE - softnose skates}

Bathyraja microtrachys (Osburn \& Nichols, 1916). Fine-spined Skate. Known to occur from Washington to "southern California" (Last et al. 2016d). Records occur to the north (Farallon Islands, CAS) and south (Guadalupe Island, Baja California, holotype, USNM). Common name follows Ebert (2003). 
Bathyraja spinosissima (Beebe and Tee-Van, 1941). Pacific White Skate. Known to occur from Oregon to Ecuador (Last et al. 2016d). Records occur to the north (off Oregon, CAS), west (Gumdrop Seamount, CAS), and south (Ecuador). Common name follows Ebert (2003).

\section{Order Stomiiformes}

Family STERNOPTYCHIDAE - marine hatchetfishes

Sternoptyx pseudobscura Baird, 1971. Highlight Hatchetfish. Records occur to the north (British Columbia: Peden 1974, Milkova et al. 2016) and south (southern California, SIO).

\section{Family STOMIIDAE - dragonfishes}

Opostomias mitsuii Imai, 1941. Pitgum Dragonfish. Records occur to the west, north, and south (USNM, UW, Peden 1974, Milkova et al. 2016). Ranges across the temperate north Pacific; rare in the CalCOFI sampling area (Moser 1996).

\section{Order Myctophiformes}

\section{Family NEOSCOPELIDAE - blackchins}

Scopelengys tristis Alcock, 1890. Pacific Blackchin. Records occur to the north (British Columbia, Milkova et al. 2016; Oregon, UW records) and south (southern California, SIO).

\section{Family MYCTOPHIDAE - lanternfishes}

Lampadena urophaos Paxton, 1963. Sunbeam Lampfish. Type locality is southern California. Occurs between $25^{\circ} \mathrm{N}$ and $42^{\circ} \mathrm{N}$ in the central and eastern Pacific (Nafpaktitis and Paxton 1968).

\section{Order Scorpaeniformes}

\section{Family SCORPAENIDAE - scorpionfishes}

Sebastes alutus (Gilbert, 1890). Pacific Ocean Perch. Literature indicates the species occurs from central Baja to Bering Sea and Japan (Miller and Lea 1972, Love et al. 2002, Butler et al. 2012). Records nearest to MBNMS occur off Point Arena (north) and SE of Santa Cruz Island (south). 


\section{Family COTTIDAE - sculpins}

Paricelinus hopliticus Eigenmann and Eigenmann, 1889. Thornback Sculpin. Records occur to the north (Cordell Bank, CAS; and Farallon Islands, SIO) and south (Point Loma, SIO). Considered a rare fish, occurring in deep, rocky bottom habitats.

\section{Order Perciformes}

\section{Family ZOARCIDAE - eelpouts}

Lycodapus endemoscotus Peden and Anderson, 1978. Deepwater Eelpout. Known from lower continental slope waters between British Columbia to northern Mexico and the Gulf of California in depths from 933 to 2,225 m (Peden and Anderson 1978). Those habitats are found in MBNMS; and based on published range, is likely to occur in MBNMS (personal communication, ME Anderson). Common name follows Mecklenburg et al. (2002).

Pachycara gymninium Anderson and Peden, 1988. Nakednape Eelpout. Known from off Queen Charlotte Islands, British Columbia to off Guadalupe Island, Mexico and in the Gulf of California in depths from 1,829 to 3,219 $\mathrm{m}$ over brown and green mud bottoms (Anderson and Peden 1988). Those habitats are found in MBNMS; and based on published range, is likely to occur in MBNMS (pers. comm. ME Anderson). Records off California include Mendocino Ridge $(3,225 \mathrm{~m})$ and off Cordell Bank (2,707-3,219 m). Common name follows Mecklenburg et al. (2002).

Pachycara lepinium Anderson and Peden, 1988. Scalynape Eelpout. Known from off Queen Charlotte Islands, British Columbia to off Guadalupe Island, Mexico in depths from 1,728 to 2,970 m over brown and green mud bottoms (Anderson and Peden 1988). Those habitats are found in MBNMS; and based on published range, is likely to occur in MBNMS (pers. comm. ME Anderson). Records off California include off Cape Mendocino (2,940 m), off Farallon Islands (1,800 m), and San Clemente Basin (1,829-2,027 m). Common name follows Mecklenburg et al. (2002).

\section{Family STICHAEIDAE - pricklebacks}

Esselenichthys laurae (Follett \& Anderson, 1990). Twoline Prickleback. Follett and Anderson (1990) described the species from 15 juvenile specimens captured between Southeast Farallon Island, California, and Punta Banda, Baja California Norte, Mexico. The Southeast Farallon Island specimens (CAS) were collected from pigeon guillemot nests, but their condition indicates they were taken locally (Follett and Anderson 1990).

Poroclinus rothrocki Bean, 1890. Whitebarred Prickleback. Northeast Pacific records include Alaska, Washington, Oregon, northern California (Crescent City, Eureka, Fort Bragg; LACM), and one record in San Diego (CAS-SU). 


\section{Family GOBIIDAE - gobies}

Ilypnus gilberti (Eigenmann \& Eigenmann, 1889). Cheekspot Goby. Occurs in mud flats of bays (Miller and Lea 1972); considered an estuarine species. No records from Elkhorn Slough; however, found to the north and south of MBNMS (e.g., Tomales Bay, San Francisco Bay, southern California).

\section{Family GEMPYLIDAE - snake mackerels}

Lepidocybium flavobrunneum (Smith, 1843). Escolar. Although range occurs from Peru to Washington (Miller and Lea 1972), it is rare, found deep, and is a more tropical species.

\section{Family ISTIOPHORIDAE - billfishes}

Tetrapturus angustirostris Tanaka, 1915. Shortbill Spearfish. Found in eastern Pacific from Chile to 40 miles west of Cape Mendocino (Miller and Lea 1972). Radovich 1961 reported a sport-catch record off Morro Bay in Aug 1959 (El Niño). In addition, two collected outside of MBNMS: 100 miles offshore, halfway between San Francisco and Morro Bay (SIO, Jul 1959); and $\sim 120$ miles west of Monterey (CAS, K Peterson, Sept 1981).

\section{Order Pleuronectiformes}

\section{Family PLEURONECTIDAE - righteye flounders}

Reinhardtius hippoglossoides (Walbaum, 1792). Greenland Halibut. Found in the northern part of the Pacific, from Sagami Bay northward, in the Sea of Japan, the Okhotsk Sea, the Bering Sea, and off the Pacific coast of North America south to Mexico (Dyck et al. 2007). It is uncommon off California (Miller and Lea 1972); several records off Eureka. The Mexican record (SIO) was captured during 1962; a cold-water year.

\section{Order Tetradontiformes}

\section{Family TETRAODONTIDAE - puffers}

Lagocephalus lagocephalus (Linnaeus, 1758). Oceanic Puffer. Published range in eastern Pacific is Galapagos Islands to Alder Creek Beach, Mendocino County; rare off California (Miller and Lea 1972). Five California museum specimens exist (4 at southern CA, 1 at Mendocino during 1958 El Niño, CAS). Typically occurs in warmer seas; however, can move north during El Niño years. 


\section{Acknowledgements}

For use of online databases, we thank the California Academy of Sciences (Ichthyology Collection Database); Scripps Institution of Oceanography (Marine Vertebrate Collection); Smithsonian National Museum of Natural History (Fish Collection database); University of Washington Burke Museum (Ichthyology Collections Database); Museum of Comparative Zoology, Harvard University; and FishNet2 portal (including: Academy of Natural Sciences of Drexel University; University of Kansas Biodiversity Institute; Los Angeles County Museum of Natural History; North Carolina Museum of Natural Sciences; Oregon State University, Department of Fisheries and Wildlife; Ohio State University, Museum of Biological Diversity, Museum of Zoology, Fish Division; Santa Barbara Museum of Natural History; University of Alaska Museum of the North, Fairbanks; University of Colorado Museum of Natural History; University of Florida, Florida Museum of Natural History; University of Michigan Museum of Zoology; Smithsonian National Museum of Natural History). For access to, and assistance with, institution records and specimens, we thank David Catania, Mysi Hoang, and Jon Fong (California Academy of Sciences); William Watson (Southwest Fisheries Science Center/NOAA Fisheries); Rick Feeney and Christine Thacker (Los Angeles County Museum of Natural History); Scott Hamilton and Catarina Pien (Moss Landing Marine Labortories); H.J. Walker, Philip Hastings, Cynthia Klepadlo, and Ben Frable (Scripps Institution of Oceanography); Linda Kuhnz (Monterey Bay Aquarium Research Institute); Theodore W. Pietsch and Katherine P. Maslenikov (University of Washington Burke Museum); William Leo Smith (University of Kansas); and Ken Oda and Paul Reilly (California Department of Fish and Wildlife). For specimens collected during 2015 midwater trawl survey, we thank the scientists and crew aboard NOAA Ship Bell M. Shimada. For visual record information, we thank Nancy Jacobsen Stout, Linda Kuhnz, Lonny Lundsten, Brian Schlining, Kyra Schlining, and Susan von Thun (Monterey Bay Aquarium Research Institute); Andrew Morgan and Joe Welsh (Monterey Bay Aquarium); Megan Bassett and James Lindholm (CSU Monterey Bay); Steve Lonhart and Chad King (Monterey Bay National Marine Sanctuary); and Alan Baldridge (Hopkins Marine Station). For review and advice on specific groups, we thank M. Eric Anderson (Zoarcidae; South African Institute for Aquatic Biodiversity), and Kerstin Wasson (Elkhorn Slough fishes). For access to historic literature and library records, we thank the Biodiversity Heritage Library, NOAA Seattle Library staff, Katie Lage (Moss Landing Marine Laboratories), and Don Kohrs (Harold A. Miller Library at Hopkins Marine Station, Stanford University). For map figure, we thank Sophie De Beukelaer (Lynker Technologies LLC). For review and comments on manuscript, we thank Larry Allen, Gregor M. Cailliet, Andrew DeVogelaere, Steve Lonhart, and H.J. Walker. Funding was provided by NOAA's Monterey Bay National Marine Sanctuary and Save The Earth Foundation, and supported by sanctuary leadership William Douros, Paul Michel, and Andrew DeVogelaere. The scientific results and conclusions, as well as any views or opinions expressed herein, are those of the authors and do not necessarily reflect the views of NOAA or the Department of Commerce. This publication does not constitute an endorsement of any commercial product or intend to be an opinion beyond scientific or other results obtained by the National Oceanic and Atmospheric Administration (NOAA). 


\section{References}

Ahlstrom EH, Moser HG, Cohen DM (1984) Argentinoidei: Development and relationships. In: Moser HG, Richards WJ, Cohen DM, Fahay MP, Kendall AW Jr, Richardson SL (Eds) Ontogeny and Systematics of Fishes. American Society of Ichthyologists and Herpetologists, Special Publication No 1: 155-169. http://swfsc.noaa.gov/publications/ CR/1984/8402.PDF

Allen MJ, Smith GB (1988) Atlas and zoogeography of common fishes in the Bering Sea and northeastern Pacific. US Department of Commerce, NOAA Technical Report NMFS 66, 151 pp. https://spo.nmfs.noaa.gov/sites/default/files/legacy-pdfs/tr66.pdf

Anderson ME (1995) The eelpout genera Lycenchelys Gill and Taranetzella Andriashev (Teleostei: Zoarcidae) in the eastern Pacific, with descriptions of nine new species. Proceedings of the California Academy of Sciences (Series 4) 49(2): 55-113. https://www.biodiversitylibrary.org/part/52965

Anderson ME (2012) A new species of Pachycara Zugmayer (Teleostei: Zoarcidae) from off Monterey Bay, California, USA, with comments on two North Pacific Lycenchelys species. Zootaxa 3559: 39-43. https://doi.org/10.11646/zootaxa.3559.1.3

Anderson ME, Peden AE (1988) The eelpout genus Pachycara (Teleostei: Zoarcidae) in the northeastern Pacific Ocean, with descriptions of two new species. Proceedings of the California Academy of Sciences (Series 4) 46(3): 83-94. https://www.biodiversitylibrary.org/ part/53679

Anderson ME, Cailliet GM, Antrim BS (1979) Notes on some uncommon deep-sea fishes from the Monterey Bay Area, California. California Fish and Game 65(4): 256-264. https://www.biodiversitylibrary.org/item/61897

Anderson ME, Fedorov VV (2004) Family Zoarcidae Swainson 1839 eelpouts. California Academy of Sciences Annotated Checklists of Fishes 34: 1-58. https://www.calacademy. org/sites/default/files/assets/docs/zoarcidae.pdf

Andrews CB (1927) Albacore caught near Monterey. California Fish and Game 13(2): 141142. https://www.biodiversitylibrary.org/item/70558

Ayres WO (1854) Description of new fishes from California. (Minutes of Academy meetings were printed in "The Pacific" (a newspaper) shortly after each meeting. New species date to publication in The Pacific. Dates of publication are given in each species account). The Pacific [newspaper] vol. 3 and 4 (thru no. 6). [Also as Proceedings of the California Academy of Natural Sciences vol. 1 (nos. 3-22).] https://www.biodiversitylibrary.org/ item/54191

Ayres WO (1860a) Descriptions of new species of fishes. Proceedings of the California Academy of Sciences (Series 1) 2: 60-64. http://www.biodiversitylibrary.org/page/3836984

Ayres WO (1860b) [Description of Fishes]. Proceedings of the California Academy of Sciences (Series 1) 2: 77-86. http://www.biodiversitylibrary.org/page/3837001

Balanov A, Savinykh VF (1999) Redescription of Scopelosaurus harryi and S. adleri (Notosudidae): two valid mesopelagic species inhabiting the northern part of the Pacific Ocean. Journal of Ichthyology 39(8): 616-625. 
Balushkin AV (2012) Volodichthys gen. nov. new species of the primitive snailfish (Liparidae: Scorpaeniformes) of the Southern Hemisphere. Description of new species $V$. solovjevae sp. nov. (Cooperation Sea, the Antarctic). Journal of Ichthyology 52(1): 1-10. https://doi. org/10.1134/S0032945212010018

Barry JP (1983) Utilization of shallow marsh habitats by fishes in Elkhorn Slough, California. MSc Thesis, San Jose State University, California. http://islandora.mlml.calstate.edu/ islandora/object/islandora\%3A461

Bean TH (1894) Description of a new blennioid fish from California. Proceedings of the United States National Museum 16(967): 699-701. https://doi.org/10.5479/si.00963801.16967.699

Bolin RL (1938) Bathylagus wesethi, a new Argentinid fish from California. California Fish and Game 24(1): 66-68. https://www.biodiversitylibrary.org/item/70695

Bolin RL (1939) A new stomiatoid fish from California. Copeia 1939(1): 39-41. https://doi. org/10.2307/1436014

Bolin RL (1952) Two unusual records of marine fishes at Monterey, California. California Fish and Game 38(2): 209-210. https://www.biodiversitylibrary.org/item/61382

Boulenger GA (1895) Catalogue of the fishes in the British Museum. Catalogue of the perciform fishes in the British Museum. Second edition. Vol. I. Catalogue of the fishes in the British Museum (2 ${ }^{\text {nd }}$ edn) Vol. 1, 394 pp. [pls. 1-15] https://babel.hathitrust.org/cgi/pt?id =uc1.31822032980716; ;iew=1 up;seq=5

Bradbury MG, Cohen DM (1958) An illustration and a new record of the North Pacific bathypelagic fish Macropinna microstoma. Stanford Ichthyological Bulletin 7(3): 57-59.

Brown JA (2006) Classification of juvenile flatfishes to estuarine and coastal habitats based on the elemental composition of otoliths. Estuarine, Coastal and Shelf Science 66: 594-611. https://doi.org/10.1016/j.ecss.2005.11.005

Burton EJ, Kuhnz LA, DeVogelaere AP, Barry JP (2017) Sur Ridge Field Guide: Monterey Bay National Marine Sanctuary. Marine Sanctuaries Conservation Series ONMS-17-10. US Department of Commerce, National Oceanic and Atmospheric Administration, Office of National Marine Sanctuaries, Silver Spring, 122 pp. https://sanctuaries.noaa.gov/science/ conservation/sur-ridge-field-guide-monterey-bay-national-mairne-sanctuary.html

Burton EJ, Lundsten L (2008) Davidson Seamount Taxonomic Guide. Marine Sanctuaries Conservation Series ONMS-08-08. United States Department of Commerce, National Oceanic and Atmospheric Administration, Office of National Marine Sanctuaries, Silver Spring, 145 pp. Available at: https://sanctuaries.noaa.gov/science/conservation/pdfs/taxonomic.pdf

Butler JL, Love MS, Laidig TE (2012) A guide to the rockfishes, thornyheads, and scorpionfishes of the Northeast Pacific. University of California Press, Berkeley, 185 pp.

Caffrey JM, Broenkow WW (2002) Hydrography. In: Caffrey JM, Brown M, Tyler WB (Eds) Changes in a California Estuary: An Ecosystem Profile of Elkhorn Slough. Monterey Bay Aquarium Foundation. Monterey, California, 29-42. http://digital.mlml.calstate.edu/islandora/object/islandora $\% 3 \mathrm{~A} 2447$

Cailliet GM, Anderson ME (1975) Occurrence of the prowfish Zaprora silenus Jordan, 1896 in Monterey Bay, California. California Fish and Game 61(1): 60-62. https://www.biodiversitylibrary.org/item/61825 
Cailliet GM, Lea, RN (1977) Abundance of the "rare" zoarcid, Maynea californica Gilbert, 1915, in the Monterey Canyon, Monterey Bay, California. California Fish and Game 63(4): 253-261. https://www.biodiversitylibrary.org/item/61890

Cailliet GM, Antrim B, Ambrose D, Pace S, Stevenson M (1977) Species composition, abundance and ecological studies of fishes, larval fishes, and zooplankton in Elkhorn Slough. In: Nybakken JW, Cailliet GM, Broenkow WW (Eds) Ecological and hydrographic studies of Elkhorn Slough, Moss Landing Harbor and nearshore coastal waters, July 1974 to June 1976. Moss Landing Marine Laboratories, Moss Landing, California, 216-386. http:// aquaticcommons.org/2607/

Carlisle AB, Litvin SY, Hazen EL, Madigan DJ, Goldman KJ, Lea RN, Block BA (2015) Reconstructing habitat use by juvenile salmon sharks links upwelling to strandings in the California Current. Marine Ecology Progress Series 525: 217-228. https://doi.org/10.3354/ meps 11183

Carvalho MR de, Last PR, Séret B (2016) Torpedo Rays. Family Torpedinidae. In: Last PR, White WT, Carvalho MR de, Séret B, Stehmann MFW, Naylor GJP (Eds) Rays of the World. Cornell University Press, Ithaca, New York, 184-203. http://www.publish.csiro. $\mathrm{au} / \mathrm{book} / 7053 /$

Castro JI (2011) The Sharks of North America. Oxford University Press, New York, 613 pp.

CDFW [California Department of Fish and Wildlife] (2017) Fishing and Diving Records. https://www.wildlife.ca.gov/Fishing/Records [accessed 9 Feb 2017]

Cohen DM, Inada T, Iwamoto T, Scialabba N (1990) Gadiform Fishes of the World (Order Gadiformes). An annotated and illustrated catalogue of cods, hakes, grenadiers and other gadiform fishes known to date. FAO Fisheries Synopsis 125(10): 1-442.

Collette BB, Aaland CR (1996) Revision of the frigate tunas (Scombridae, Auxis), with descriptions of two new subspecies from the eastern Pacific. Fishery Bulletin 94(3): 423-441. https://www.st.nmfs.noaa.gov/spo/FishBull/943/collette.pdf

Compagno LJV (1988) Sharks of the Order Carcharhiniformes. Princeton University Press, Princeton, New Jersey, $572 \mathrm{pp}$.

Cooper JG (1863) On new genera and species of Californian fishes - No. II. Proceedings of the California Academy of Sciences (Series 1) 3: 93-97. http://www.biodiversitylibrary.org/ page/3148002

Cox KW (1948) Sablefish run at Monterey Bay. California Fish and Game 34(1): 37. https:// www.biodiversitylibrary.org/item/70838

Cramer F (1895) On the cranial characters of the genus Sebastodes (rock-fish). Proceedings of the California Academy of Sciences (Series 2) 5: 573-610. http://www.biodiversitylibrary. org/page/32283261

Didier DA, Séret B (2002) Chimaeroid fishes of New Caledonia with description of a new species of Hydrolagus (Chondrichthyes, Holocephali). Cybium 26(3): 225-233.

Dill WA, Cordone AJ (1997) History and status of introduced fishes in California, 1871-1996. California Department of Fish and Game, Fish Bulletin 178: 1-414. https://escholarship. org/uc/item/5rm0h8qg

Dyck M, Warkentin PH, Treble MA (2007) A bibliography on the Greenland halibut, Reinhardtius hippoglossoides (a.k.a. Greenland turbot) 19;6-2005. Canadian Technical Report of 
Fisheries and Aquatic Sciences 2683, 309 pp. http://publications.gc.ca/site/eng/422643/ publication.html

Ebeling AW (1962) Melamphaidae I. Systematics and zoogeography of the species in the bathypelagic fish genus Melamphaes Günther. Dana-Report 58(1): 1-164.

Ebert DA (2003) The Sharks, Rays and Chimaeras of California. University of California Press, Berkeley, 284 pp. https:/www.ucpress.edu/book.php?isbn=9780520234840

Ebert DA, Mollet HF, Baldridge A, Thomas T, Forney KA, Ripley WE (2004) Occurrence of the whale shark, Rhincodon typus Smith 1828, in California waters. Northwestern Naturalist 85(1): 26-28. https://doi.org/10.1898/1051-1733(2004)085<0026:OOTWSR>2.0.CO;2

Eigenmann CH, Beeson CH (1894) Pteropodus dallii sp. nov. American Naturalist 28(325): 66. https://doi.org/10.1086/275871

Eschmeyer WN, Fricke R, van der Laan R (2017) Catalog of Fishes: Genera, Species, References. http://researcharchive.calacademy.org/research/ichthyology/catalog/fishcatmain.asp [accessed 31 May 2017]

Eschmeyer WN, Herald ES, Hammann H (1983) A Field Guide to Pacific Coast Fishes. Houghton Mifflin Company, Boston, 336 pp.

Fast TN (1957) The occurrence of the deep-sea anglerfish, Cryptopsaras couesii, in Monterey Bay, California. Copeia 1957(3): 237-240. https://doi.org/10.2307/1439375

Fitch JE (1949) Some unusual occurrences of fish on the Pacific coast. California Fish and Game 35(1): 41-49. https://www.biodiversitylibrary.org/item/61373

Fitch JE (1951) Notes on the squaretail, Tetragonurus cuvieri. California Fish and Game 37(1): 55-59. https://www.biodiversitylibrary.org/item/61378

Fitch JE (1963) A review of the fishes of the genus Pleuronichthys. Los Angeles County Museum Contributions in Science No. 76: 33 pp. https://www.biodiversitylibrary.org/part/241024

Fitch JE (1964) Sebastodes phillipsi, a new scorpaenid fish from Californian waters. Copeia 1964(3): 525-529. https://doi.org/10.2307/1441517

Fitch JE, Lavenberg RJ (1968) Deep-Water Fishes of California. University of California Press, Berkeley, 155 pp. https://archive.org/details/deepwaterteleost00fitc

Follett WI (1948) A northerly record of Polydactylus approximans (Lay and Bennett), a polynemid fish of the Pacific Coast of tropical America. Copeia 1948(1): 34-40. https://doi. org/10.2307/1438788

Follett WI (1966) Man-eater of the California coast. Pacific Discovery 19(1): 18-22.

Follett WI, Anderson ME (1990) Esselenia, a new genus of pricklebacks (Teleostei: Stichaeidae), with two new species from California and Baja California Norte. Copeia 1990(1): 147-163. https://doi.org/10.2307/1445831

Follett WI, Powell DC (1988) Ernogrammus walkeri, a new species of prickleback (Pisces: Stichaeidae) from south-central California. Copeia 1988(1): 135-152. https://doi. org/10.2307/1445933

Frable BW, Wagman DW, Frierson TN, Aguilar A, Sidlauskas BL (2015) A new species of Sebastes (Scorpaeniformes: Sebastidae) from the northeastern Pacific, with a redescription of the blue rockfish, S. mystinus (Jordan \& Gilbert, 1881). Fishery Bulletin 113(4): 355-377. https://doi.org/10.7755/FB.113.4.1 
Frey HW (1962) A range extension for the Mexican scad to Monterey Bay, California. California Fish and Game 48(3): 210-211. https://www.biodiversitylibrary.org/item/63636

Gaither MR, Violi B, Gray HWI, Neat F, Drazen JC, Grubbs RD, Roa-Varón A, Sutton T, Hoelzel AR (2016) Depth as a driver of evolution in the deep sea: Insights from grenadiers (Gadiformes: Macrouridae) of the genus Coryphaenoides. Molecular Phylogenetics and Evolution 104: 73-82. https://doi.org/10.1016/j.ympev.2016.07.027

Gibbs MA (1991) Notes on the distribution and morphology of the rubynose brotula (Cataetyx rubrirostris) off central California. California Fish and Game 77(3): 149-152. https:// www.biodiversitylibrary.org/item/72536

Gilbert CH (1896) The ichthyological collections of the steamer Albatross during the years 1890 and 1891. United States Commission of Fish and Fisheries, Report of the Commissioner 19(1893): 393-476. https://babel.hathitrust.org/cgi/pt?id=hvd.hwfibi;view=1 up;seq=9

Gilbert CH (1904) Notes on fishes from the Pacific coast of North America. Proceedings of the California Academy of Sciences (Series 3) 3(9): 255-271. http://www.biodiversitylibrary. org/page/31548105

Gilbert CH (1914) Two cottoid fishes from Monterey Bay, California. Proceedings of the United States National Museum 47(2049): 135-137. https://doi.org/10.5479/si.00963801.472049.135

Gilbert CH (1915) Fishes collected by the United States Fisheries steamer "Albatross" in southern California in 1904. Proceedings of the United States National Museum 48(2075): 305-380. https://doi.org/10.5479/si.00963801.48-2075.305

Girard CF (1854) Observations upon a collection of fishes made on the Pacific coast of the United States, by Lieut. W.P. Trowbridge, U.S.A., for the museum of the Smithsonian Institution. Proceedings of the Academy of Natural Sciences of Philadelphia 7: 142-156. https://repository.si.edu/handle/10088/34410

Girard CF (1855) Characteristics of some cartilaginous fishes of the Pacific coast of North America. Proceedings of the Academy of Natural Sciences of Philadelphia 7(6): 196-197. https://www.biodiversitylibrary.org/part/7975

Girard CF (1858a) Fishes. In: Part IV: General report upon zoology of the several Pacific railroad routes, 1857. In: Reports of explorations and surveys, to ascertain the most practicable and economical route for a railroad from the Mississippi River to the Pacific Ocean, v. 10. Beverley Tucker, Washington, DC, 400 pp. https://www.biodiversitylibrary.org/item/57065

Girard CF (1858b) Notice upon new genera and new species of marine and fresh-water fishes from western North America. Proceedings of the Academy of Natural Sciences of Philadelphia 9(15): 200-202. https://www.biodiversitylibrary.org/item/30013

Gotshall DW (1961) Observations on a die-off of molas (Mola mola) in Monterey Bay. California Fish and Game 47(4): 339-341. https://www.biodiversitylibrary.org/item/61609

Greeley AW (1898) Oligocottus snyderi. In: Jordan DS, Evermann BW (Eds) The fishes of North and Middle America: a descriptive catalogue of the species of fish-like vertebrates found in the waters of North America north of the Isthmus of Panama. Part III. Bulletin of the United States National Museum No. 47: 2871. https:/www.biodiversitylibrary.org/ item/32368 
Greeley AW (1899) Notes on the tide-pool fishes of California, with a description of four new species. Bulletin of the United States Fish Commission 19: 7-20. https://www.biodiversitylibrary.org/item/211768

Guerrero J, Kvitek RG (1996) Monterey Bay National Marine Sanctuary Site Characterization. https://montereybay.noaa.gov/sitechar/welcome.html

Günther A (1861) A preliminary synopsis of the labroid genera. Annals and Magazine of Natural History (Series 3) 8(47): 382-389. https://doi.org/10.1080/00222936108697435

Herald ES (1953) The 1952 shark derbies at Elkhorn Slough, Monterey Bay, and at Coyote Point, San Francisco Bay. California Fish and Game 39(2): 237-243. https://www.biodiversitylibrary.org/item/61449

Herald ES, Dempster RP (1952) The 1951 shark derby at Elkhorn Slough, California. California Fish and Game 38(1): 133-134. https://www.biodiversitylibrary.org/item/61381

Hill KT, Haight DR (1985) Northward range extension for the striped marlin. California Fish and Game 71(3): 185-187. https://www.biodiversitylibrary.org/item/63729

Hoff GR (1999) Range extensions of 3 species of macrourids from the west coast of North America. California Fish and Game 85(3): 113-117.

Hoff GR, Buckley TW, Drazen JC, Duncan KM (2000) Biology and ecology of Nezumia liolepis and $N$. stelgidolepis from the west coast of North America. Journal of Fish Biology 57(3): 662-680. https://doi.org/10.1111/j.1095-8649.2000.tb00267.x

Hopkirk JD (1965) Records of yellow and spotted snake-eels (Genus Ophichthus) from San Francisco Bay, California. California Fish and Game 51(3): 183-186.

Horn MH, Allen LG, Lea RN (2006) Biogeography. In: Allen LG, Pondella DJ II, Horn $\mathrm{MH}$ (Eds) The Ecology of Marine Fishes: California and Adjacent Waters. University of California Press, Berkeley, California, 3-25. https://doi.org/10.1525/califor$\mathrm{nia} / 9780520246539.003 .0001$

Hubbs CL (1926) Notes on the gobioid fishes of California, with descriptions of two new genera. Occasional Papers of the Museum of Zoology University of Michigan 169: 1-6. https://deepblue.lib.umich.edu/handle/2027.42/56608

Hubbs CL (1927) Notes on the blennioid fishes of western North America. Papers of the Michigan Academy of Science Arts and Letters 7(1926): 351-394. https://archive.org/ details/in.ernet.dli.2015.26778

Hubbs CL (1953) Revision and systematic position of the blenniid fishes of the genus Neoclinus. Copeia 1953(1): 11-23. https://doi.org/10.2307/1440237

Hubbs CL, Follett WI, Dempster LJ (1979) List of the fishes of California. Occasional Papers of the California Academy of Sciences, San Francisco, California 133: 1-51. https://www. biodiversitylibrary.org/item/35725

Humphreys RL Jr, Winans GA, Tagami DT (1989) Synonomy and life history of the North Pacific Pelagic Armorhead, Pseudopentaceros wheeleri Hardy (Pisces: Pentacerotidae). Copeia 1989(1): 142-153. https://doi.org/10.2307/1445615

Hyde JR, Underkoffler KE, Sundberg MA (2014) DNA barcoding provides support for a cryptic species complex within the globally distributed and fishery important opah (Lampris guttatus). Molecular Ecology Resources 14: 1239-1247. https://doi.org/10.1111/17550998.12268 
Iglesias SP, Lecointre G, Sellos DY (2005) Extensive paraphylies within sharks of the order Carcharhiniformes inferred from nuclear and mitochondrial genes. Molecular Phylogenetics and Evolution 34: 569-583. https://doi.org/10.1016/j.ympev.2004.10.022

Jacobson Stout N, Kuhnz L, Lundsten L, Schlining B, Schlining K, von Thun S (Eds) (2017) The Deep-Sea Guide. Monterey Bay Aquarium Research Institute. http://dsg.mbari.org [accessed 8 Jun 2017]

Jordan DS (1887a) The Fisheries of the Pacific Coast. United States Commission of Fish and Fisheries, The Fisheries and Fishery Industries of the United States, Section II, Part XVI, 589-629. https://www.biodiversitylibrary.org/item/77475

Jordan DS (1887b) Coast of California. In: The Whale Fishery. 1. History and present condition of the fishery. United States Commission of Fish and Fisheries, The Fisheries and Fishery Industries of the United States, Section V, Volume II, Part XV, 52-61. https:// www.biodiversitylibrary.org/item/77552

Jordan DS (1896) Notes on fishes, little known or new to science. Proceedings of the California Academy of Sciences (Series 2) 6: 201-244. http://www.biodiversitylibrary.org/ page/16072474

Jordan DS, Eigenmann CH (1890) A review of the genera and species of Serranidae found in the waters of America and Europe. Bulletin of the United States Fish Commission 8(1888): 329-441. https://hdl.handle.net/2027/inu.30000112165737

Jordan DS, Evermann BW (1896a) A check-list of the fishes and fish-like vertebrates of North and Middle America. United States Commission of Fish and Fisheries, Report of the Commissioner, vol. 21 (for 1895), Appendix 5: 207-584. https://archive.org/details/achecklistfishe00evergoog

Jordan DS, Evermann BW (1896b) The fishes of North and Middle America: a descriptive catalogue of the species of fish-like vertebrates found in the waters of North America, north of the Isthmus of Panama. Part I. Bulletin of the United States National Museum No. 47, 1240 pp. https://www.biodiversitylibrary.org/item/32405

Jordan DS, Evermann BW (1900) The fishes of North and Middle America: a descriptive catalogue of the species of fish-like vertebrates found in the waters of North America, north of the Isthmus of Panama. Part IV. Bulletin of the United States National Museum No. 47, 3137-3313. [pls. 1-392] https://doi.org/10.5962/bhl.title.39720

Jordan DS, Gilbert CH (1880a) Notes on sharks from the coast of California. Proceedings of the United States National Museum 3(118): 51-52. https://doi.org/10.5479/si.00963801.3118.51

Jordan DS, Gilbert CH (1880b) Description of a new species of Sebastichthys (Sebastichthys miniatus), from Monterey Bay, California. Proceedings of the United States National Museum 3(125): 70-72. https://doi.org/10.5479/si.00963801.3-125.70

Jordan DS, Gilbert CH (1880c) Description of a new species of "rock-fish" (Sebastichthys carnatus), from the coast of California. Proceedings of the United States National Museum 3(126): 73-75. https://doi.org/10.5479/si.00963801.3-126.73

Jordan DS, Gilbert CH (1880d) Description of a new species of ray (Raia stellulata) from Monterey, California. Proceedings of the United States National Museum 3(129): 133-135. https://doi.org/10.5479/si.00963801.3-129.133 
Jordan DS, Gilbert CH (1880e) Descriptions of new species of Xiphister and Apodichthys, from Monterey, California. Proceedings of the United States National Museum 3(130): 135140. https://doi.org/10.5479/si.00963801.3-130.135

Jordan DS, Gilbert CH (1880f) Description of two new species of Sebastichthys (Sebastichthys entomelas and Sebastichthys rhodochloris), from Monterey Bay, California. Proceedings of the United States National Museum 3(132): 142-146. https://doi.org/10.5479/ si.00963801.3-132.142

Jordan DS, Gilbert CH (1880g) Description of a new agonoid fish (Brachyopsis xyosternus), from Monterey Bay, California. Proceedings of the United States National Museum 3(135): 152-154. https://doi.org/10.5479/si.00963801.3-135.152

Jordan DS, Gilbert CH (1880h) Description of a new species of ray, Raia rhina, from the coast of California. Proceedings of the United States National Museum 3(141): 251-253. https://doi.org/10.5479/si.00963801.3-141.251

Jordan DS, Gilbert CH (1880i) Description of seven new species of sebastoid fishes, from the coast of California. Proceedings of the United States National Museum 3(150): 287-298. https://doi.org/10.5479/si.00963801.3-150.287

Jordan DS, Gilbert CH (1880j) Description of a new embiotocoid (Abeona aurora), from Monterey, California, with notes on a related species. Proceedings of the United States National Museum 3(151): 299-301. https://doi.org/10.5479/si.00963801.3-151.299

Jordan DS, Gilbert CH (1880k) Description of a new embiotocoid fish (Ditrema atripes), from the coast of California. Proceedings of the United States National Museum 3(156): 320 322. https://doi.org/10.5479/si.00963801.3-156.320

Jordan DS, Gilbert CH (1880l) Description of a new scorpaenoid fish (Sebastichthys proriger), from Monterey Bay, California. Proceedings of the United States National Museum 3(161): 327-329. https://doi.org/10.5479/si.00963801.3-161.327

Jordan DS, Gilbert CH (1881) Description of a new species of "rock-fish" (Sebastichthys chrysomelas), from the coast of California. Proceedings of the United States National Museum 3(176): 465-466. https://doi.org/10.5479/si.00963801.3-176.465

Jordan DS, Gilbert CH (1882) Notes of the fishes of the Pacific Coast of the United States. Proceedings of the United States National Museum 4(1881): 29-70. https://doi.org/10.5479/ si.00963801.4-191.29

Kamikawa DJ, Stevenson DE (2010) New records of Aldrovandia oleosa (Notacanthiformes: Halosauridae) from the eastern North Pacific Ocean. California Fish and Game 96(3): 216-220. https://nrm.dfg.ca.gov/FileHandler.ashx?DocumentID=47311 \&inline=1

Kells V, Rocha LA, Allen LG (2016) A field guide to coastal fishes from Alaska to California. Johns Hopkins University Press, Baltimore, 366 pp. https://jhupbooks.press.jhu.edu/content/field-guide-coastal-fishes-0

Kline DE, Donlou NE, Lea RN, Lindholm JB, Shester GG (2013) Records of the longfin gunnel, Pholis clemensi, from California with a southern range extension to Point Lobos, California. Marine Biodiversity Records 6(e10): 1-4. https://doi.org/10.1017/ S1755267212001169

Kotlyar AN (2004) Family Melamphaidae Gill 1893 - bigscales. California Academy of Sciences Annotated Checklists of Fishes 29: 1-11. https://www.calacademy.org/sites/default/ files/assets/docs/melamphaidae.pdf 
Knudsen SW, Clements KD (2013) Revision of the fish family Kyphosidae (Teleostei: Perciformes). Zootaxa 3751(1): 1-101. https://doi.org/10.11646/zootaxa.3751.1.1

Knudsen SW, Clements KD (2016) World-wide species distributions in the family Kyphosidae (Teleostei: Perciformes). Molecular Phylogenetics and Evolution 101: 252-266. https:// doi.org/10.1016/j.ympev.2016.04.037

Last PR, Séret B, Naylor GJP (2016a) A new species of guitarfish, Rhinobatos borneensis sp. nov. with a redefinition of the family-level classification in the order Rhinopristiformes (Chondrichthyes: Batoidea). Zootaxa 4117(4): 451-475. https://doi.org/10.11646/ zootaxa.4117.4.1

Last PR, Stehmann MF, Séret B, Weigmann S (2016b) Softnose Skates. Family Arhynchobatidae. In: Last PR, White WT, de Carvalho MR, Séret B, Stehmann MFW, Naylor GJP (Eds) Rays of the World. Cornell University Press, Ithaca, New York, 364-472. https://doi. org/10.1071/9780643109148

Last PR, Weigmann S, Yang L (2016c) Changes to the nomenclature of the skates (Chondrichthyes: Rajiformes). In: Last PR, Yearsley GK (Eds) Rays of the World: Supplementary Information. CSIRO Special Publication, 11-34. http://www.publish.csiro.au/book/7053/

Last PR, White WT, de Carvalho MR, Séret B, Stehmann MFW, Naylor GJP (Eds) (2016d) Rays of the World. Cornell University Press, Ithaca, 800 pp. https://doi. org/10.1071/9780643109148

Lauth RR (2000) The 1999 Pacific west coast upper continental slope trawl survey of groundfish resources off Washington, Oregon, and California: Estimates of distribution, abundance, and length composition. US Department of Commerce, NOAA Technical Memorandum NMFS-AFSC-115, 287 pp. https://www.afsc.noaa.gov/techmemos/nmfs-afsc-115.htm

Lauth RR (2001) The 2000 Pacific west coast upper continental slope trawl survey of groundfish resources off Washington, Oregon, and California: Estimates of distribution, abundance, and length composition. US Department of Commerce, NOAA Technical Memorandum NMFS-AFSC-120, 284 pp. https://www.afsc.noaa.gov/techmemos/nmfsafsc-120.htm

Lay GT, Bennett ET (1839) Fishes. In: Bechey FW (Ed) The zoology of Captain Beechey's voyage, compiled from the collections and notes made by Captain Beechey, the officers and naturalist of the expedition, during a voyage to the Pacific and Behring's Straits performed in his Majesty's Ship Blossom, under the command of Captain F.W. Beechey, R.N., F.R.S., \&c. \&c. in the years 1825, 26, 27, and 28. H.G. Bohn, London, 41-75. https://archive. org/details/zoologyofcaptain00beec

Lea RN (1972) Southern geographical records for four surfperches, family Embiotocidae, with notes on a population resurgence of the sharpnose seaperch. California Fish and Game 58 (1): 27-31. https://www.biodiversitylibrary.org/item/61759

Lea RN (1983) Sebastodes atrorubens Gilbert, 1898, a junior synonym of Sebastes atrovirens (Jordan and Gilbert, 1880), with notes on individual variation in the species. Bulletin Southern California Academy of Sciences 82(3): 147-149. http://biodiversitylibrary.org/ page/34407012

Lea RN (1988) Family Himantolophidae added to the ichthyofauna of the temperate eastern north Pacific. California Fish and Game 74(3): 172-185. https://www.biodiversitylibrary. org/item/72474 
Lea RN (1997) Central California Marine Sport Fish Project: Marine Biodiversity Study and CPFV Sampling Study. California Department of Fish and Game Cruise Report 97-M-9, prepared 24 November 1997, 7 pp.

Lea RN (2013) Record of the Kamchatka Flounder, Atheresthes evermanni, in California waters. Northwestern Naturalist 94(3): 244-246. https://doi.org/10.1898/13-01.1

Lea RN, Karpov KA, Quirollo LF (1989) Record of the roughscale sole, Clidoderma asperrimum, from northern California with a note on the Pacific lined sole, Achirus maxatlanus. California Fish and Game 75(4): 239-241.

Lea RN, Keating T, Van Dykhuizen G, Lehtonen PB (1984) Records of goosefishes (family: Lophiidae, genus Lophiodes) from Californian waters. California Fish and Game 70(4): 250-251. https://www.biodiversitylibrary.org/item/72410

Lea RN, Rosenblatt RH (1987) Occurrence of the family Notacanthidae (Pisces) from marine waters of California. California Fish and Game 73(1): 51-53. https://www.biodiversitylibrary.org/item/72468

Lea RN, Rosenblatt RH (2000) Observations on fishes associated with the 1997-98 El Niño off California. CalCOFI Report 41: 117-129. http://www.calcofi.org/publications/calcofireports/v41/Vol_41_Lea__Rosenblatt.pdf

Lea RN, Walker HJ Jr (1995) Record of the bigeye trevally, Caranx sexfasciatus, and Mexican lookdown, Selene brevoorti, with notes on other carangids from California. California Fish and Game 81(3): 89-95. https://www.wildlife.ca.gov/Publications/Journal/Contents

Limbaugh C (1955) Fish life in the kelp beds and the effects of kelp harvesting. University of California, Institute of Marine Resources, IMR Reference 55-9: 1-158. https://escholarship.org/uc/item/4w36x6mb

Lockington WN (1879) On a new genus and species of Scombridae. Proceedings of the Academy of Natural Sciences of Philadelphia 31: 133-136. https://www.biodiversitylibrary.org/part/84801 Lockington WN (1880) Description of a new chiroid fish, Myriolepis zonifer, from Monterey Bay, California. Proceedings of the United States National Museum 3(140): 248-251. https://doi.org/10.5479/si.00963801.140.248

Lonhart SI (2009) Natural and Climate Change Mediated Invasions. In: Rilov G, Crooks JA (Eds) Biological Invasions in Marine Ecosystems. Ecological Studies 204, Springer-Verlag, Berlin/Heidelberg, 57-69. https://doi.org/10.1007/978-3-540-79236-9_3

Longo GC, Bernardi G, Lea RN (2018) Taxonomic revisions within Embiotocidae (Teleostei, Perciformes) based on molecular phylogenetics. Zootaxa 4482(3): 591-596. https://doi. org/10.11646/zootaxa.4482.3.10

Love MS, Mecklenburg CW, Mecklenburg TA, Thorsteinson LK (2005) Resource Inventory of Marine and Estuarine Fishes of the West Coast and Alaska: A Checklist of North Pacific and Arctic Ocean Species from Baja California to the Alaska-Yukon Border. U. S. Department of the Interior, U. S. Geological Survey, Biological Resources Division, Seattle, Washington, 98104, OCS Study MMS 2005-030 and USGS/NBII 2005-001. http:// www.lovelab.id.ucsb.edu/checklist.html.

Love MS, Yoklavich MM, Thorsteinson LK (2002) The rockfishes of the Northeast Pacific. University of California Press, Berkeley, California, 405 pp. https://www.ucpress.edu/ op.php?isbn=9780520234383 
Lundsten L, Johnson SB, Cailliet GM, DeVogelaere AP, Clague DA (2012) Morphological, molecular, and in situ behavioral observations of the rare deep-sea anglerfish Chaunacops coloratus (Garman 1899), order Lophiiformes, in the eastern North Pacific. Deep Sea Research I 68: 46-53. https://doi.org/10.1016/j.dsr.2012.05.012

Lundsten L, McClain CR, Barry JP, Cailliet GM, Clague DA, DeVogelaere AP (2009) Ichthyofauna on three seamounts off southern and central California, USA. Marine Ecology Progress Series 389: 223-232. https://doi.org/10.3354/meps08181

Martinez-Takeshita N, Purcell CM, Chabot CL, Craig MT, Paterson CN, Hyde JR, Allen LG (2015) A tale of three tails: cryptic speciation in a globally distributed marine fish of the genus Seriola. Copeia 103(2): 357-368. https://doi.org/10.1643/CI-124-224

Mecklenburg CW, Mecklenburg TA, Thorsteinson LK (2002) Fishes of Alaska. American Fisheries Society, Bethesda, Maryland, 1116 pp.

Meek SE, Pierson CJ (1895) Description of a new species of Gobiesox from Monterey Bay, California. Proceedings of the California Academy of Sciences (Series 2) 5: 571-572. http:// www.biodiversitylibrary.org/page/32283259

Milkova V, Hanke G, Gillespie G, Fong K, Boutillier J, Bedard J (2016) Range records for ten species of stomiiform, aulopiform, and myctophiform fishes in British Columbia, Canada. Northwestern Naturalist 97(2): 113-123. https://doi.org/10.1898/NWN15-11.1

Miller DJ, Gotshall D (1965) Ocean Sportfish Catch and Effort from Oregon to Point Arguello, California, July 1, 1957-June 30, 1961. California Department of Fish and Game, Fish Bulletin 130, 135 pp. http://content.cdlib.org/ark:/13030/kt1g5001fm

Miller DJ, Lea RN (1972) Guide to the coastal marine fishes of California. California Department of Fish and Game, Fish Bulletin 157: 1-249. http://content.cdlib.org/ark:/13030/ kt896nb2qd

Mollet HF (2002) Distribution of the pelagic stingray, Dasyatis violacea (Bonaparte, 1832), off California, Central America, and worldwide. Marine and Freshwater Research 53: 525530. https://doi.org/10.1071/MF02010

Monterey Bay Aquarium (1999) Natural History of the Monterey Bay National Marine Sanctuary. Monterey Bay Aquarium Foundation, Monterey, 299 pp.

Monterey County Herald (1997) Unusual catch in local waters: Fishermen take mahi mahi off Point Pinos. [2 Sept 1997; Section B; Michelle Maitre]

Monterey Herald (2018) 'Tsunami' fish shows up in Monterey Bay. [10 Dec 2018; Rodrigo Pérez Ortega] https:/www.montereyherald.com/2018/12/10/tsunami-fish-shows-up-inmonterey-bay/

Morita T (1999) Molecular phylogenetic relationships of the deep-sea fish genus Coryphaenoides (Gadiformes: Macrouridae) based on mitochondrial DNA. Molecular Phylogenetics and Evolution 13(3): 447-454. https://doi.org/10.1006/mpev.1999.0661

Moser HG (1996) Melanostomiidae: Scaleless dragonfishes. In: Moser HG (Ed) The early stages of fishes in the California Current region. California Cooperative Oceanic Fisheries Investigations, Atlas 33, Allen Press, Lawrence, Kansas, 308-319. http://calcofi.org/publications/atlases/CalCOFI_Atlas_33.pdf

Moser HG, Butler JL (1996) Microstomatidae: Argentines and pencilfishes. In: Moser HG (Ed) The early stages of fishes in the California Current region. California Cooperative 
Oceanic Fisheries Investigations, Atlas 33, Allen Press, Lawrence, Kansas, 208-215. http:// calcofi.org/publications/atlases/CalCOFI_Atlas_33.pdf

Moyle PB (2002) Inland Fishes of California. University of California Press, Berkeley, California, 502 pp. https:/www.ucpress.edu/book.php?isbn=9780520227545

Nafpaktitis BG, Paxton JR (1968) Review of the lanternfish genus Lampadena with a description of a new species. Los Angeles County Museum Contributions in Science No. 138, 29 pp. https://www.biodiversitylibrary.org/part/241127

Nelson JS, (2006) Fishes of the World ( $4^{\text {th }}$ edn). John Wiley \& Sons, New York, 624 pp. https:// www.wiley.com/en-us/Fishes+of+the+World\%2C+4th+Edition-p-9780471250319

Nelson JS, Crossman EJ, Espinosa-Pérez H, Findley LT, Gilbert CR, Lea RN, Williams JD (2004) Common and scientific names of fishes from the United States, Canada, and Mexico (6 ${ }^{\text {th }}$ edn). American Fisheries Society, Special Publication 29, Bethesda, 386 pp. https:// fisheries.org/bookstore/all-titles/special-publications/x51029xm/

Nelson JS, Grande TC, Wilson MVH (2016) Fishes of the World (5 $5^{\text {th }}$ edn). John Wiley \& Sons, New Jersey, 752 pp. https://doi.org/10.1002/9781119174844

Nelson L (1986) A range extension of Phidiana sternsi (Cockerell, 1901) (Gastropoda: Nudibranchia). The Veliger 29(2): 240. https:/www.biodiversitylibrary.org/part/96977

NOAA Earth System Research Laboratory (2019) El Niño Southern Oscillation (ENSO): ENSO Index Dashboard. https://www.esrl.noaa.gov/psd/enso/dashboard.html [accessed 2016-2019] NOAA Fisheries (2016) Species in the Spotlight, Priority Actions: 2016-2020, Central California Coast Coho Salmon Oncorhynchus kisutch. Central California Coast Coho 5-Year Action Plan, 17 pp. http://www.nmfs.noaa.gov/stories/2015/07/spotlight_central_ca_cohosalmon.html

Norris KS (1963) The functions of temperature in the ecology of the percoid fish Girella nigricans (Ayres). Ecological Monographs 33(1): 23-62. https://doi.org/10.2307/1948476

Null J (2019) El Niño and La Niña years and intensities based on Oceanic Niño Index (ONI). https://www.ggweather.com/enso/oni.htm [accessed 2016-2019]

Orr JW (2012) Two new species of snailfishes of the genus Careproctus (Scorpaeniformes: Liparidae) from the Bering Sea and eastern North Pacific Ocean, with a redescription of Careproctus ovigerus. Copeia 2012(2): 257-265. https://doi.org/10.1643/CI-11-046

Orr JW, Hawkins S (2008) Species of the rougheye rockfish complex: resurrection of Sebastes melanostictus (Matsubara, 1934) and a redescription of Sebastes aleutianus (Jordan and Evermann, 1898) (Teleostei: Scorpaeniformes). Fishery Bulletin 106(2): 111-134. https:// www.st.nmfs.noaa.gov/spo/FishBull/1062/orr.pdf

Page LM, Espinosa-Pérez H, Findley LT, Gilbert CR, Lea RN, Mandrak NE, Mayden RL, Nelson JS (2013) Common and scientific names of fishes from the United States, Canada, and Mexico ( $7^{\text {th }}$ edn). American Fisheries Society, Special Publication 34, Bethesda, 384 pp. https://fisheries.org/bookstore/all-titles/special-publications/51034c/

Paulin, CD (1989) Review of the morid genera Gadella, Physiculus, and Salilota (Teleostei: Gadiformes) with descriptions of seven new species. New Zealand Journal Zoology 16: 93-133. https://doi.org/10.1080/03014223.1989.10423706

Peden AE (1974) Rare fishes including first records of thirteen species from British Columbia. Syesis 7: 47-62. https://publications.royalbcmuseum.bc.ca/product/syesis-vol-7/ 
Peden AE, Anderson ME (1978) A systematic review of the fish genus Lycodapus (Zoarcidae) with descriptions of two new species. Canadian Journal of Zoology 56(9): 1925-1961. https://doi.org/10.1139/z78-262

Phillips JB (1930a) Wolf fish captured at Monterey. California Fish and Game 16(3): 267-268. https://www.biodiversitylibrary.org/item/70562

Phillips JB (1930b) Large black sea bass caught in Monterey Bay. California Fish and Game 16(3): 268. https://www.biodiversitylibrary.org/item/70562

Phillips JB (1931a) Albacore at Monterey. California Fish and Game 17(1): 85. https://www. biodiversitylibrary.org/item/70691

Phillips JB (1931b) Another wolf fish taken at Monterey. California Fish and Game 17(1): 85-86. https://www.biodiversitylibrary.org/item/70691

Phillips JB (1932a) Unusually good fishing in and off Monterey Bay. California Fish and Game 18(1): 21-24. https://www.biodiversitylibrary.org/item/70692

Phillips JB (1932b) Monterey Spanish mackerel landed in Monterey. California Fish and Game 18(1): 99. https://www.biodiversitylibrary.org/item/70692

Phillips JB (1932c) Wolf-fish taken at Monterey. California Fish and Game 18(1): 99. https:// www.biodiversitylibrary.org/item/70692

Phillips JB (1932d) Ribbon-fish taken at Monterey. California Fish and Game 18(1): 99-100. https:/www.biodiversitylibrary.org/item/70692

Phillips JB (1935) A large northern halibut in Monterey. California Fish and Game 21(3): 262. https://www.biodiversitylibrary.org/item/70693

Phillips JB (1936) Big-eyed bass taken at Monterey. California Fish and Game 22(1): 48-49. https://archive.org/details/californiafishga22_1936cali

Phillips JB (1937) Record mackerel taken at Monterey. California Fish and Game 23(4): 337. https://archive.org/details/californiafishga231937cali

Phillips JB (1940) A California needlefish recorded at Monterey. California Fish and Game 26(3): 289.

Phillips JB (1942a) Wall-eyed pollock caught in Monterey Bay. California Fish and Game 28(3): 155-156. https://www.biodiversitylibrary.org/item/70701

Phillips JB (1942b) Tongue Sole in Monterey Bay. California Fish and Game 28(3): 156. https://www.biodiversitylibrary.org/item/70701

Phillips JB (1943a) Grunion in Monterey Bay. California Fish and Game 29(2): 82. https:// www.biodiversitylibrary.org/item/70703

Phillips JB (1943b) Another wall-eyed pollock at Monterey. California Fish and Game 29(2): 83. https://www.biodiversitylibrary.org/item/70703

Phillips JB (1945) Two unusual flatfishes from Monterey Bay. California Fish and Game 31(4): 210-211. https://www.biodiversitylibrary.org/item/70831

Phillips JB (1948) Basking shark fishery revived in California. California Fish and Game 34(1): 11-23. https://www.biodiversitylibrary.org/item/70838

Phillips JB (1951a) Pacific cod off central California. California Fish and Game 37(3): 351. https:/www.biodiversitylibrary.org/item/61379

Phillips JB (1951b) Round herring off central California. California Fish and Game 37(4): 512. https://www.biodiversitylibrary.org/item/61380 
Phillips JB (1953) Additional Pacific cod taken off central California. California Fish and Game 39(4): 559. https://www.biodiversitylibrary.org/item/61450

Phillips JB (1954) Another large black sea bass caught in Monterey Bay. California Fish and Game 40(3): 339. https://www.biodiversitylibrary.org/item/61453

Phillips JB (1957) A review of the rockfishes of California (Family Scorpaenidae). California Department of Fish and Game, Fish Bulletin 104: 1-158. http://content.cdlib.org/ ark:/13030/kt729005d8

Phillips JB (1958) Southerly occurrences of three northern species of fish during 1957, a warmwater year on the California coast. California Fish and Game 44(4): 349-350. https:// www.biodiversitylibrary.org/item/61532

Phillips JB (1961) Range extensions for two California fishes, with a note on a rare fish. California Fish and Game 47(4): 418. https://www.biodiversitylibrary.org/item/61609

Phillips JB (1963) A fantail sole, Xystreurys liolepis, in Monterey Bay. California Fish and Game 49(3): 209. https://www.biodiversitylibrary.org/item/61613

Phillips JB (1965a) Northern range extension for the zebraperch, Hermosilla azurea Jenkins and Evermann. California Fish and Game 51(1): 55-56. https://www.biodiversitylibrary. org/item/61618

Phillips JB (1965b) Range extension for the bigmouth sole, Hippoglossina stomata. California Fish and Game 51(2): 125-126. https://www.biodiversitylibrary.org/item/61619

Phillips JB (1966) Skilfish, Erilepis zonifer (Lockington), in Californian and Pacific Northwest waters. California Fish and Game 52(3): 151-156. https:/www.biodiversitylibrary.org/ item/61681

Phillips JB (1967) A longfin sanddab, Citharichthys xanthostigma, and a skilfish, Erilepis zonifer, taken in Monterey Bay. California Fish and Game 53(4): 297-298. https://www.biodiversitylibrary.org/item/61686

Prokofiev AM (2014) Swallowers (Chiasmodontidae) of the East Pacific. Journal of Ichthyology 54(9): 631-641. https://doi.org/10.1134/S0032945214060137

Prokofiev AM, Kukuev EI (2009) Systematics and distribution of black swallowers of the genus Chiasmodon (Perciformes: Chiasmodontidae). Journal of Ichthyology 49(10): 899-939. https://doi.org/10.1134/S0032945209100063

Quirollo LF, Dinnel PA (1975) Latitudinal range extensions for yellow and spotted snake eels (genus Opichthus). California Fish and Game 61(3): 156-157. https://www.biodiversitylibrary.org/item/61827

Radovich J (1961) Relationships of some marine organisms of the Northeast Pacific to water temperatures particularly during 1957 through 1959. Fish Bulletin 112: 1-61. http://content.cdlib.org/ark:/13030/kt4r29n72m

Randall JE, DiBattista JD (2012) Etrumeus makiawa, a new species of round herring (Clupeidae: Dussumierinae) from the Hawaiian Islands. Pacific Science 66(1): 97-110. https:// doi.org/10.2984/66.1.6

Reichert AN, Lundsten L, Ebert DA (2016) First North Pacific records of the pointy nosed blue chimaera, Hydrolagus cf. trolli (Chondrichthyes: Chimaeriformes: Chimaeridae). Marine Biodiversity Records 9: 90. https://doi.org/10.1186/s41200-016-0095-5 
Robison BH, Reisenbichler KR (2008) Macropinna microstoma and the paradox of its tubular eyes. Copeia 2008(4): 780-784. https://doi.org/10.1643/CG-07-082

Russell BC (2003) Synodontidae and Bathysauridae. In: Carpenter KE (Ed), The living marine resources of the Western Central Atlantic. Volume 2: Bony fishes part 1 (Acipenseridae to Grammatidae). FAO species identification guide for fishery purposes and American Society of Ichthyologist and Herpetologists Special Publication No. 5, FAO, Rome, 923-932. http://www.fao.org/docrep/009/y4161e/y4161e00.htm

Sabaj MH (2016) Standard symbolic codes for institutional resource collections in herpetology and ichthyology: an Online Reference. Version 6.5 (16 August 2016). American Society of Ichthyologists and Herpetologists, Washington, DC. http://www.asih.org/resources/ standard-symbolic-codes-institutional-resource-collections-herpetology-ichthyology

Sandknop EM, Watson W (1996) Melamphaidae: Bigscales. In: Moser HG (Ed) The early stages of fishes in the California Current region. California Cooperative Oceanic Fisheries Investigations, Atlas 33, Allen Press, Lawrence, Kansas, 692-711. http://calcofi.org/publications/atlases/CalCOFI_Atlas_33.pdf

Sato T, Nakabo T (2002) Paraulopidae and Paraulopus, a new family and genus of aulopiform fishes with revised relationships within the order. Ichthyological Research 49(1): 25-46. https://doi.org/10.1007/s102280200004

Scofield NB (1916) The humpback and dog salmon taken in San Lorenzo River. California Fish and Game 2: 41. https://www.biodiversitylibrary.org/item/70694

Scofield NB (1917) Basking Shark taken in Monterey Bay. California Fish and Game 3(3): 137. https:/www.biodiversitylibrary.org/item/63626

Séret B, Last PR, Naylor GJP (2016) Guitarfishes. Family Rhinobatidae. In: Last PR, White WT, de Carvalho MR, Séret B, Stehmann MFW, Naylor GJP (Eds) Rays of the World. Cornell University Press, Ithaca, 77-109. https://doi.org/10.1071/9780643109148

Sheiko BA, Mecklenburg CW (2004) Family Agonidae Swainson 1839 - poachers. California Academy of Sciences Annotated Checklists of Fishes No. 30: 1-27. https://www.calacademy.org/sites/default/files/assets/docs/agonidae.pdf

SIMoN (Sanctuary Integrated Monitoring Network) (2018) Photo Library. http://www.sanctuarysimon.org/photos/index.php [accessed 8 Aug 2018]

SIMoN (Sanctuary Integrated Monitoring Network) (2019) Species Database. https://sanctuarysimon.org/dbtools/species-database/

Skogsberg T (1939) The fishes of the family Sciaenidae (croakers) of California. Fish Bulletin 54: 1-62. http://content.cdlib.org/ark:/13030/kt0d5n97jz

Smith JG, Gotshall DW (1967) Northerly occurrences of kelp bass, Paralabrax clathratus (Girard), since 1959. California Fish and Game 53(1): 63. https://www.biodiversitylibrary. org/item/61683

Snyder JO (1913) Notes on Ranzania makua Jenkins and other species of fishes of rare occurrence on the California coast. Proceedings of the United States National Museum 44(1961): 455-460. https://doi.org/10.5479/si.00963801.44-1961.455

Spratt JD (1981) California grunion, Leuresthes tenuis, spawn in Monterey Bay, California. California Fish and Game 67(2): 134. https://www.biodiversitylibrary.org/item/72400 
Springer VG, Anderson ME (1997) Catalog of type specimens of recent fishes in the National Museum of Natural History, Smithsonian Institution, 8: Suborder Zoarcoidei (Anarhichadidae, Bathymasteridae, Pholidae, Ptilichthyidae, Scytalinidae, Stichaeidae, Zoarcidae). Smithsonian Contributions to Zoology No. 589, 1-27. https://doi.org/10.5479/ si.00810282.589

Starks EC (1919) Rare fish from Monterey Bay. California Fish and Game 5(1): 43. https:// www.biodiversitylibrary.org/item/53563

Starks EC (1921) The Basking Shark at Monterey. California Fish and Game 7(3): 178. https:// www.biodiversitylibrary.org/item/72466

Starnes WC (1988) Revision, phylogeny and biogeographic comments on the circumtropical marine percoid fish family Priacanthidae. Bulletin of Marine Science 43(2): 117-203. http://www.ingentaconnect.com/content/umrsmas/bullmar/1988/00000043/00000002/ $\operatorname{art} 00001$

Starr RM, Cope JM, Kerr LA (2002) Trends in fisheries and fishery resources associated with the Monterey Bay National Marine Sanctuary From 1981-2000. Publication No. T-046, California Sea Grant College Program, La Jolla, 169 pp. https://montereybay.noaa.gov/ research/techreports/trstarr2002b.html

Stein DL, Drazen JC, Schlining KL, Barry JP, Kuhnz L (2006) Snailfishes of the central California coast: video, photographic and morphological observations. Journal of Fish Biology 69(4): 970-986. https://doi.org/10.1111/j.1095-8649.2006.01167.x

Stevens DE, Kohlhorst DW (2001) Striped Bass. In: Leet WS, Dewees CM, Klingbeil R, Larson EJ (Eds) California’s Living Marine Resources: A Status Report. University of California, 460-464. https://www.wildlife.ca.gov/Conservation/Marine/Status/2001

Stevenson DE, Matarese AC (2005) The ronquils: a review of the North Pacific fish family Bathymasteridae (Actinopterygii: Perciformes: Zoarcoidei). Proceedings of the Biological Society of Washington 118(2): 367-406. https://doi.org/10.2988/0006-324X(2005)118[ 367:TRAROT]2.0.CO;2

Ta N, Miller JA, Chapman JW, Pleus AE, Calvanese T, Miller-Morgan T, Burke J, Carlton JT (2018) The Western Pacific barred knifejaw, Oplegnathus fasciatus (Temminck \& Schlegel, 1844) (Pisces: Oplegnathidae), arriving with tsunami debris on the Pacific coast of North America. Aquatic Invastions 13(1): 179-186. https://doi.org/10.3391/ai.2018.13.1.14

Thompson WF (1920) The grunion at Monterey. California Fish and Game 6(3): 130. https:// www.biodiversitylibrary.org/item/53566

Thompson WF (1921a) The basking shark at Monterey. California Fish and Game 7(3): 178. https://www.biodiversitylibrary.org/item/72466

Thompson WF (1921b) A rag fish at Monterey. California Fish and Game 7(3): 179. https:// www.biodiversitylibrary.org/item/72466

Underkoffler KE, Luers MA, Hyde JR, Craig MT (2018) A taxonomic review of Lampris guttatus (Brünnich 1788) (Lampridiformes; Lampridae) with descriptions of three new species. Zootaxa 4413(3): 551-565. https://doi.org/10.11646/zootaxa.4413.3.9

Varoujean DH (1972) The reoccurrence of the California scorpionfish, Scorpaena guttata Girard, in Monterey Bay. California Fish and Game 58(3): 238-239. https://www.biodiversitylibrary.org/item/61760 
Voskoboinikova OS, Nazarkin MV (2017) Osteology of the Southern Ocean daggertooth (Anotopterus vorax) and status of the Anotopterus genus in the Aulopiformes order. Journal of Ichthyology 57(1): 10-19. https://doi.org/10.1134/S0032945217010131

Wallace M, Ojerholm EW, Scheiff AJ, Kinziger AP (2015) First record of striped mullet (Mugil cephalus) in Humboldt Bay, California. California Fish and Game 101(4): 286-288. https://nrm.dfg.ca.gov/FileHandler.ashx?DocumentID=113248\&inline

Weigmann S (2016) Annotated checklist of the living sharks, batoids and chimaeras (Chondrichthyes) of the world, with a focus on biogeographical diversity. Journal of Fish Biology 88(3): 837-1281. https://doi.org/10.1111/jfb.12874

Weinberg KL, Wilkins ME, Shaw FR, Zimmerman M (2002) The 2001 Pacific west coast bottom trawl survey of groundfish resources: Estimates of distribution, abundance, and length and age composition. US Department of Commerce, NOAA Technical Memorandum NMFS-AFSC-128, 140 pp. https://www.afsc.noaa.gov/techmemos/nmfs-afsc-128.htm

Wilimovsky NJ, Wilson DE (1979) A new species of Agonidae, Agonomalus mozinoi, from the west coast of North America. Syesis 11:73-79. https://publications.royalbcmuseum.bc.ca/ product/syesis-vol-11/

Wilson EO (2014) The Meaning of Human Existence. Liveright Publishing Corporation, New York, 207 pp.

Wilson RR Jr, (1994) Interrelationships of the subgenera of Coryphaenoides (Gadiformes: Macrouridae): Comparison of protein electrophoresis and peptide mapping. Copeia 1994(1): 42-50. https://doi.org/10.2307/1446669

Wilson RR Jr, Attia P (2003) Interrelationships of the subgenera of Coryphaenoides (Teleostei: Gadiformes: Macrouridae): synthesis of allozyme, peptide mapping, and DNA sequence data. Molecular Phylogenetics and Evolution 27: 343-347. https://doi.org/10.1016/ S1055-7903(02)00419-0

Wisner RL (1976) The taxonomy and distribution of lanternfishes (Family Myctophidae) of the Eastern Pacific Ocean. Navy Ocean Research and Development Activity Report No. 3, Bay St. Louis, Mississippi, 229 pp. https://doi.org/10.21236/ADA041654

Wolter K, Timlin MS (2011) El Niño/Southern Oscillation behaviour since 1871 as diagnosed in an extended multivariate ENSO index (MEI.ext). International Journal of Climatology 31(7): 1074-1087. https://doi.org/10.1002/joc.2336

Yoklavich MM, Cailliet GM, Barry JP, Ambrose DA, Antrim BS (1991) Temporal and spatial patterns in abundance and diversity of fish assemblages in Elkhorn Slough, California. Estuaries 14(4): 465-480. https://doi.org/10.2307/1352270

Yoklavich MM, Cailliet GM, Oxman DS, Barry JP, Lindquist DC (2002) Fishes. In: Caffrey JM, Brown M, Tyler B (Eds) Changes in a California Estuary: An Ecosystem Profile of Elkhorn Slough. Monterey Bay Aquarium Foundation. Monterey, California, 163-185. http://digital.mlml.calstate.edu/islandora/object/islandora\%3A2438 